Louisiana State University

LSU Digital Commons

\title{
Measuring skeletal kinematics with accelerometers on the skin surface
}

Kevin Curtis Ayer

Louisiana State University and Agricultural and Mechanical College

Follow this and additional works at: https://digitalcommons.Isu.edu/gradschool_theses

Part of the Mechanical Engineering Commons

\section{Recommended Citation}

Ayer, Kevin Curtis, "Measuring skeletal kinematics with accelerometers on the skin surface" (2007). LSU Master's Theses. 2483.

https://digitalcommons.Isu.edu/gradschool_theses/2483

This Thesis is brought to you for free and open access by the Graduate School at LSU Digital Commons. It has been accepted for inclusion in LSU Master's Theses by an authorized graduate school editor of LSU Digital Commons. For more information, please contact gradetd@lsu.edu. 


\author{
A Thesis \\ Submitted to the Graduate Faculty of the \\ Louisiana State University and \\ Agricultural and Mechanical College \\ in partial fulfillment of the \\ requirements for the degree of \\ Master of Science in Mechanical Engineering
}

In

The Department of Mechanical Engineering

by

Kevin Ayer

B.S., Louisiana State University, 2002

May, 2007 


\section{Acknowledgements}

The work presented in this thesis could not have been done if it were not for the help of so many people who have shared their time, ideas, and laughter with me.

I would first like to thank Dr. Michael Murphy for sharing his ideas, time, and support for this work as well as allowing me to explore the vast world of bodily motion, a subject that has fascinated me for many years. Dr. Lopez, thank you for opening the doors so willingly at the LSU School of Veterinary Medicine and allowing me to learn both from and with you during this research. Dr. Pang, thank you for answering even my most mundane questions and having the faith in me to help teach your undergraduate dynamics class. Dr. Waggenspack, thank you for taking time to let me bounce ideas off you and getting me to open my mind about different ones.

During the many hours I have logged in and around the $\mu$ SET lab, I have had the opportunity to work alongside some great people who have both inspired me and lifted my spirits when times were difficult. I wish to thank Chetan Ramesh, Adam Cygan, Pin-Chuan Chen, Diwakar Iyer, Michael Guillet, Estelle Evans, Eric Lewis, Anish Roychowdhury, Lucian Zigoneanu, and my sincerest friend, Byoung Hee You, who was always more than willing to help me with any trouble I may have had and for believing in me from the beginning.

Of course, this page would not be complete without mentioning my family. To my parents, Fred and Joy, who spurred my curious nature and sacrificed so much to foster my busy academic and athletic careers, I cannot thank you enough for I would not be who I am today if it were not for you two. To my brothers, Michael and Scott, you have always believed in me and supported me over the years. Thank you for being the great friends you are. Finally, to my girlfriend, Rebekah McClain, thank you for all of your support and encouragement throughout this process. It would have been a more difficult adventure without you. 


\section{Table of Contents}

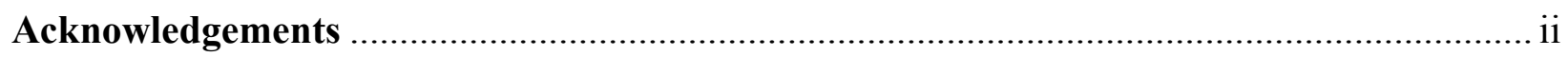

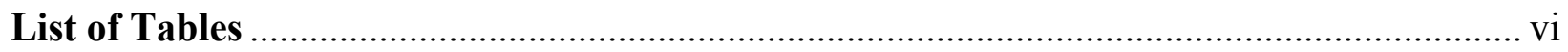

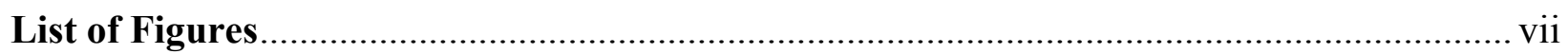

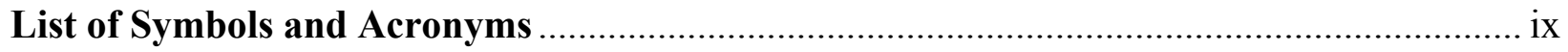

Abstract

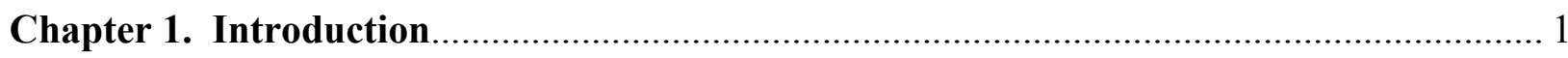

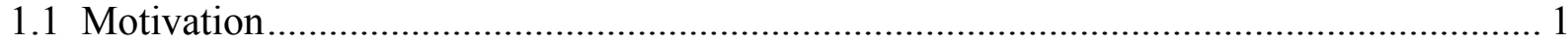

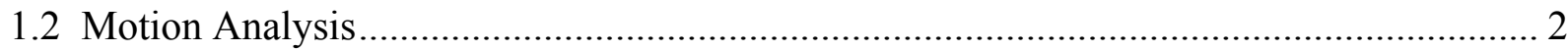

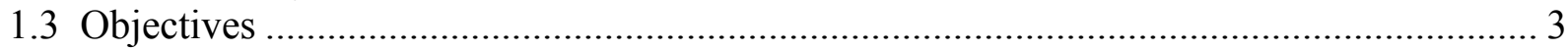

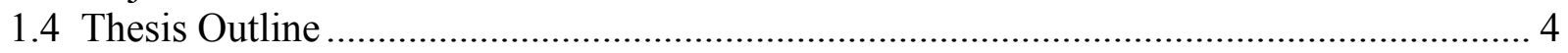

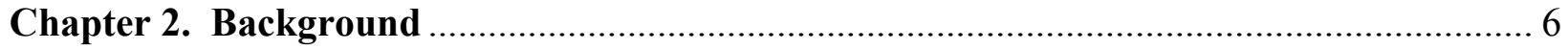

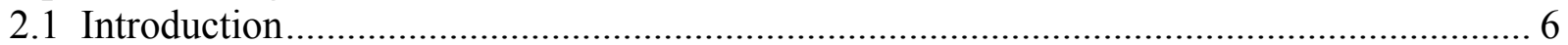

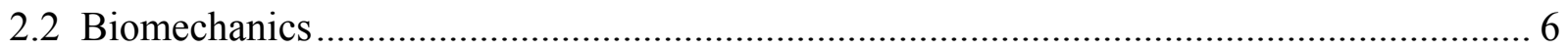

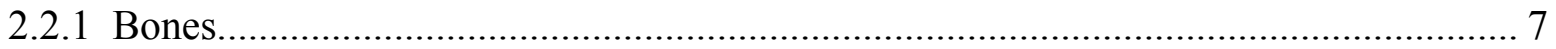

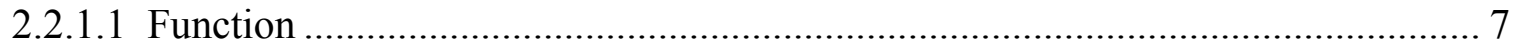

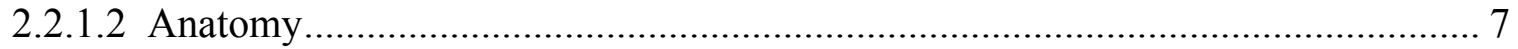

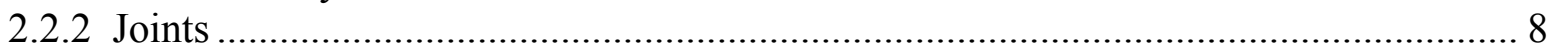

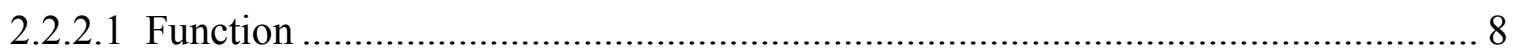

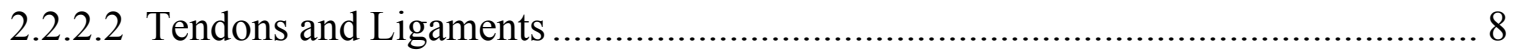

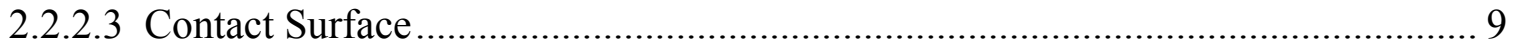

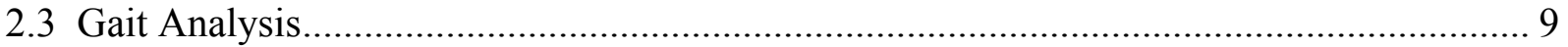

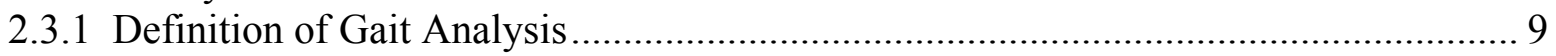

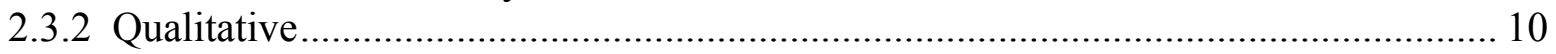

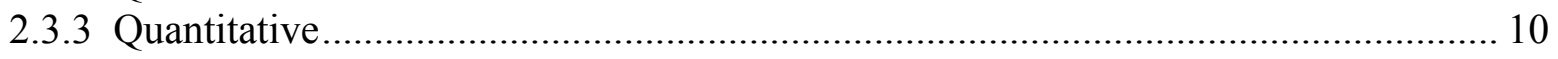

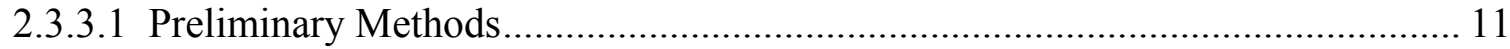

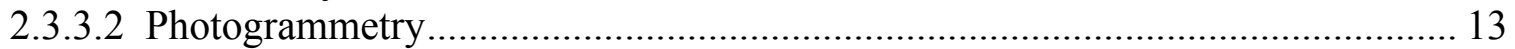

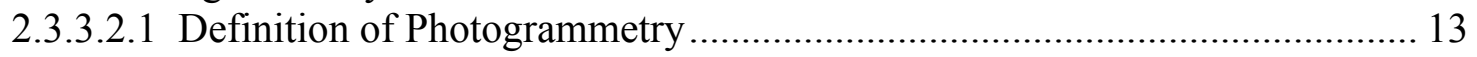

2.3.3.2.2 Camera and Marker............................................................................. 13

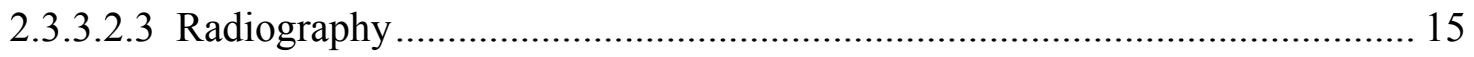

2.3.3.2.4 Magnetic Resonance Imaging ............................................................ 17

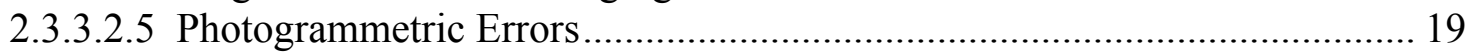

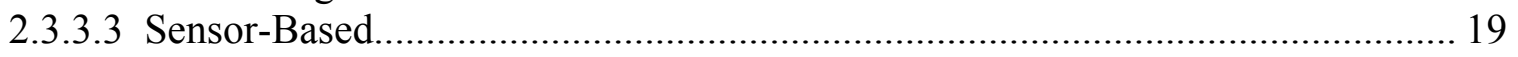

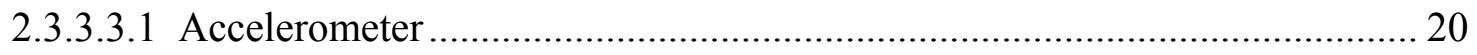

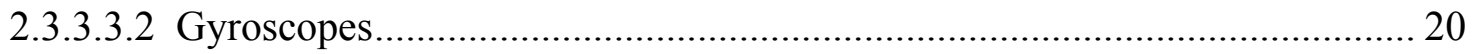

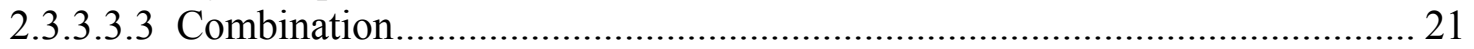

2.3.3.3.4 Magnetic Tracking ........................................................................... 22

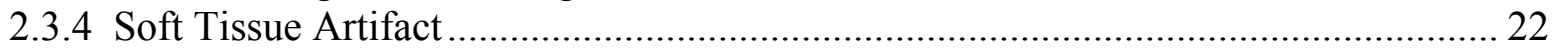

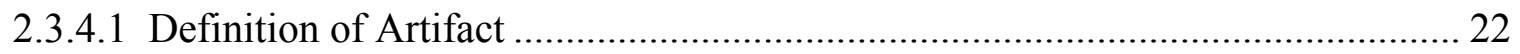




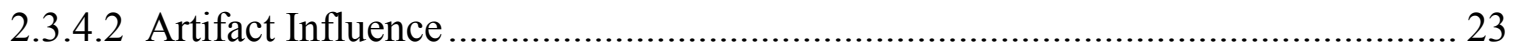

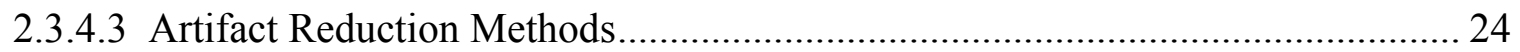

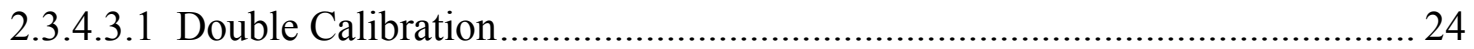

2.3.4.3.2 Point Cluster Technique.............................................................................. 25

2.3.4.3.3 Optimal Common Shape Technique............................................................ 27

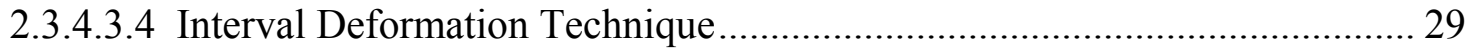

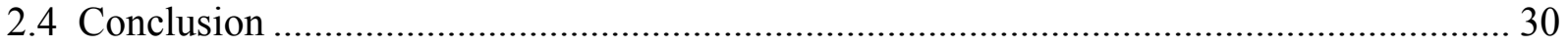

Chapter 3. Modeling the Dynamic Motion of Soft Tissue .................................................... 31

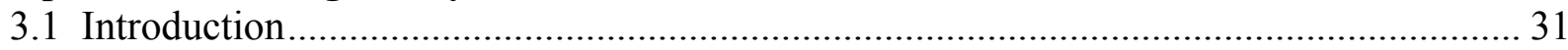

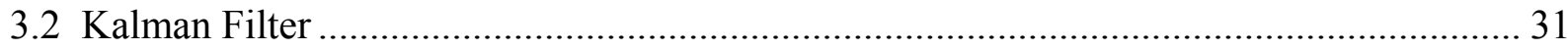

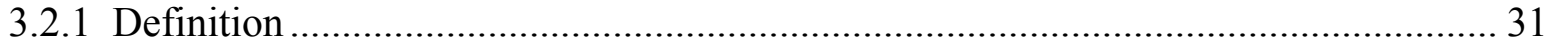

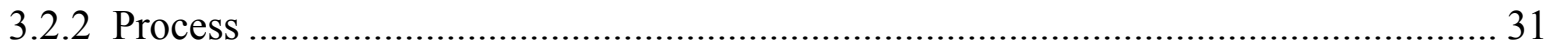

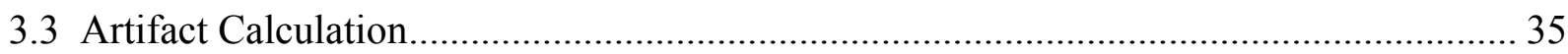

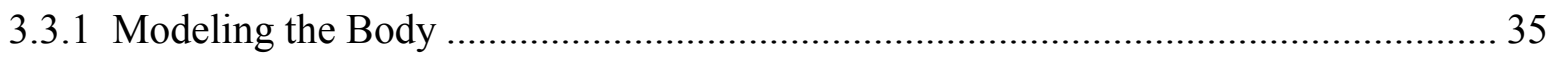

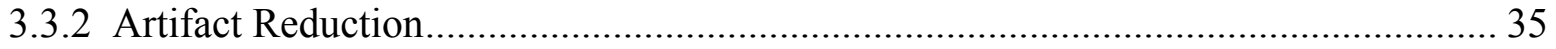

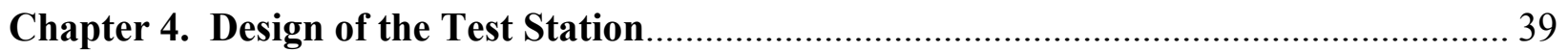

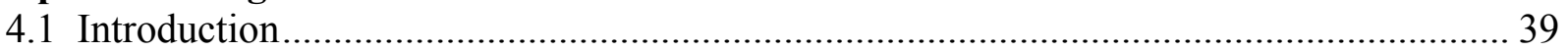

4.2 Anatomical Simulation with Cyclical, Repetitive Motion in Two Dimensions ................. 39

4.3.1 Experimental Configuration of Anatomical Representations .................................... 42

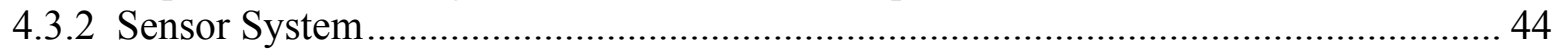

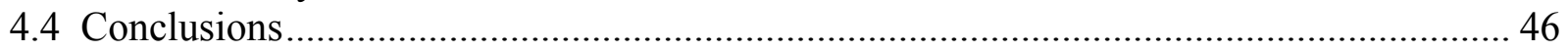

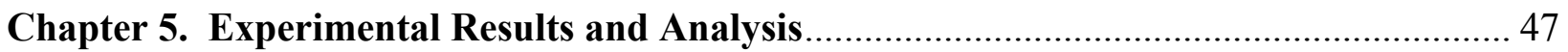

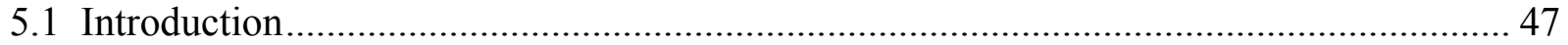

5.2 Coordinate Systems Used in Experimental Analysis .................................................... 47

5.2.1 Sensor-Based Coordinate System ............................................................................... 47

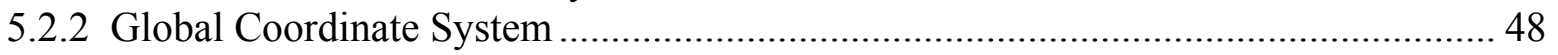

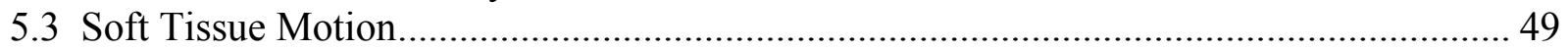

5.3.1 Collected Position Data.......................................................................................... 49

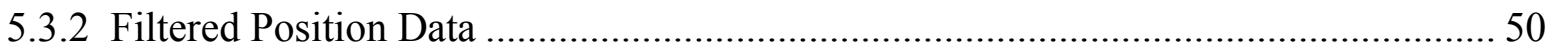

5.3.2.1 Drift and Its Effect on Position Measurements ................................................. 51

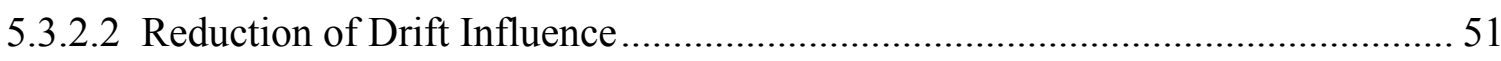

5.4 Determination of Base Motion.................................................................................... 53

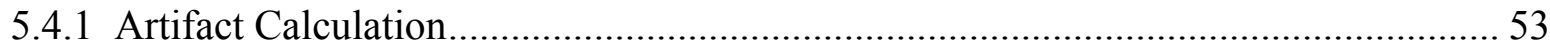

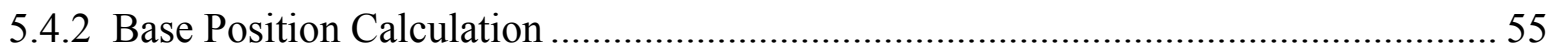

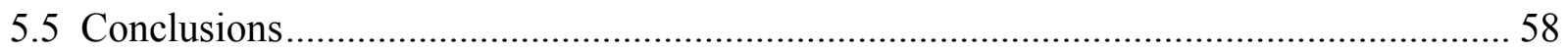

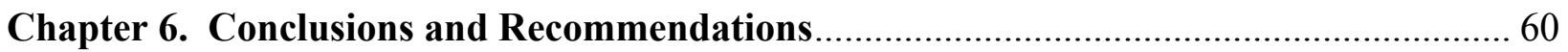

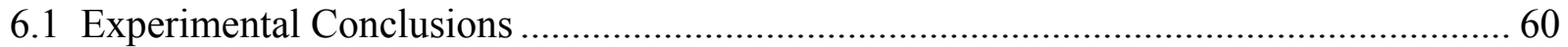

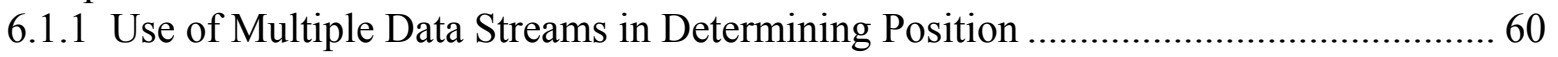

6.1.2 Using Accelerometers to Remove the Influence of Soft Tissue Motion ...................... 60

6.1.3 Conclusions Regarding Acceleration Data and Determination of Bone Data ............. 61

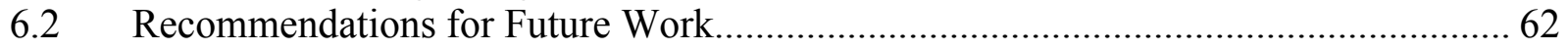


References...

Appendix A. Artifact Plots for All Six Marker Locations over Four Tests. 68

Appendix B. Base Coordinate Comparisons between Two Sensor Sets ... 80

Vita 92 


\section{List of Tables}

Table 5.1. Mean and standard deviations for artifact differences along spring axis, millimeters

Table 5.2. Artifact range and accuracy of calculated values to CODA measurements....... 55

Table 5.3. Mean and standard deviations for base $x$-coordinate differences, millimeters.. 58

Table 5.4. Mean and standard deviations for base y-coordinate differences, millimeters.. 58

Table 5.5. Base $x$-axis range and accuracy of calculated values to CODA measurements . 59

Table 5.6. Base y-axis range and accuracy of calculated values to CODA measurements . 59 


\section{List of Figures}

Figure 3.1. Conditional density of a sample measurement ........................................... 32

Figure 3.2. Calculated mean from two data sets using the Kalman filter ....................... 33

Figure 3.3. Visco-elastic model of soft tissue and a vibrating mass.............................. 35

Figure 3.4. Forces present in viso-elastic model....................................................... 36

Figure 3.5. Base and surface motion with respective displacement origin ...................... 37

Figure 4.1. Path of hind- and forelimb equine hooves in the sagittal plane

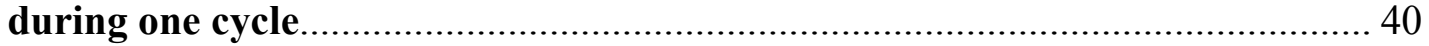

Figure 4.2. Path of hind- and forelimb equine hooves in the frontal plane ..................... 40

Figure 4.3. Representation of animal body in the test apparatus............................... 41

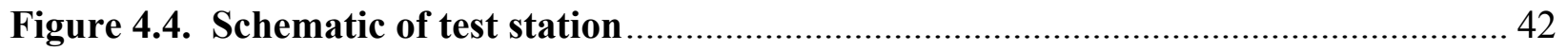

Figure 4.5. Experimental setup with simple crank mechanism ................................... 43

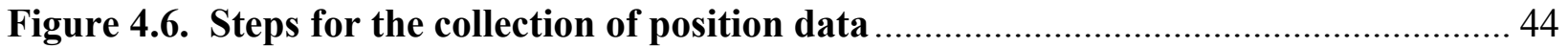

Figure 4.7. Mechanical joint and potentiometer ...................................................... 45

Figure 5.1. Accelerometer-defined coordinate system................................................ 47

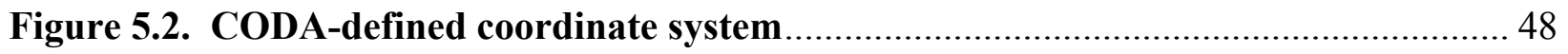

Figure 5.3. Accelerometer coordinate system transformation.................................... 49

Figure 5.4. CODA coordinate system transformation...................................................... 50

Figure 5.5. Mass position in the $x$ - and $y$-directions for position 2 in test 1 .................. 51

Figure 5.6. Mass $x$ - and $y$-positions without accelerometer data for position 2 in test 1 .... 53

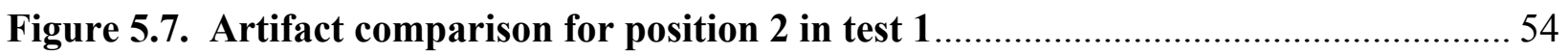

Figure 5.8. Mean and standard deviations in artifact values for position 2 in

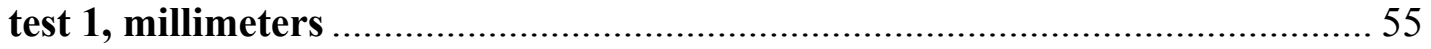

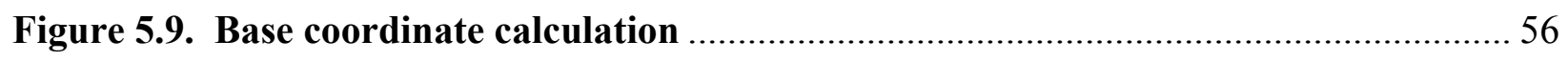


Figure 5.10. Artificial relocation of base markers with vertical offsets.

Figure 5.11. Relocation of base marker vertically below sprung marker 57

Figure 5.12. Base position in the $x$ - and $y$-directions for position 2 in test 1 57 


\section{List of Symbols and Acronyms}

\begin{tabular}{|c|c|}
\hline$\hat{x}, \mu$ & Optimal calculated variable estimate in Kalman filter \\
\hline$\sigma, \sigma_{z_{1}}, \sigma_{z_{2}}$ & Standard deviations of calculated estimate and two measurements \\
\hline & in Kalman filter \\
\hline$z_{1}, z_{2}$ & Measurements entering Kalman filter \\
\hline K & Kalman gain \\
\hline $\mathrm{F}$ & External force on tissue mass element \\
\hline M & Soft tissue mass \\
\hline $\mathrm{k}$ & Soft tissue spring constant \\
\hline $\mathrm{c}$ & Soft tissue damping coefficient \\
\hline$\ddot{x}, \dot{x}, x$ & Mass axial acceleration, velocity, and position \\
\hline$\rho$ & Mass axial displacement from rest \\
\hline$z$ & Position of base of mass \\
\hline$\ddot{y}, \dot{y}, y$ & Acceleration, velocity and position of mass relative to base \\
\hline$\omega$ & Soft tissue natural frequency \\
\hline$x_{A}, y_{A}$ & Accelerometer coordinate system axes \\
\hline$x_{C}, y_{C}$ & CODA coordinate system axes \\
\hline$x_{G}, y_{G}$ & Global coordinate system axes \\
\hline$\theta_{J o \text { int }}$ & Angle of pendulums from vertical \\
\hline$R_{\text {Pot. }}$ & Potentiometer wiper resistance \\
\hline$V_{D A Q}$ & Voltage from potentiometer wiper \\
\hline$V_{o}$ & Initial potentiometer wiper voltage \\
\hline
\end{tabular}




$\begin{array}{ll}V_{\mathrm{Re} f .} & \text { Reference voltage } \\ z_{x}, z_{y} & \text { Base positions in global coordinate system } \\ x_{K F}, y_{K F} & \text { Coordinates of mass determined by Kalman filter in global } \\ & \text { coordinate system } \\ \rho_{o} & \text { Initial distance separating mass and base } \\ \Delta y_{G, o} & \text { Vertical separation of CODA markers on mass and base at rest } \\ \Delta y_{G, o B a s e} & \text { Global y coordinates of base CODA marker at rest } \\ \Delta y_{G, o S p r u n g} & \text { Global y coordinates of sprung CODA marker at rest }\end{array}$




\begin{abstract}
The most common motion analysis method uses cameras to track the position of markers on bodily surfaces over time. Although each species has a common skeletal frame to reference recorded motions, the soft tissue covering each is not rigid. Markers, therefore, experience motion relative to the bone and do not accurately portray underlying bone activity. This limits clinical use of motion studies and the understanding of joint motion.

Use of MEMS accelerometers for removing soft tissue artifact, motion relative to the bone, from surface measurements and determining the position of the underlying bone was investigated. An animal limb was modeled experimentally as a double pendulum with soft tissue as sprung masses with motions perpendicular to the pendulums. Horizontal motion was cycled at the top joint with a $25 \mathrm{~cm}$ stroke. Position data obtained from the mass with a Codamotion ${ }^{\mathrm{TM}}$ system and integrated accelerometer data were combined in a Kalman filter to determine global position. Acceleration data in the sensor coordinate system determined tissue artifact and were compared to measurements using CODA markers on the mass and pendulum. Removing artifact from mass position estimated pendulum position over time.

In determining mass position, integrated accelerometer data experienced drift, deviating from reasonable values and were determined impractical for Kalman filter input. This led to using only the CODA-determined position as the true position.

Accelerometer artifacts resulted in mean differences with the CODA markers of less than $1 \mathrm{~mm}$ over $3 \mathrm{~cm}$ displacements excluding a mass with mechanical difficulties. The largest mean difference across four tests was $0.66 \mathrm{~mm}$, which is 96.17 percent accurate. Mean differences between base positions collected from accelerometers and CODA markers were found for the
\end{abstract}


global $\mathrm{x}$ and y directions. Maximum deviations were $1.64 \mathrm{~mm}$ and $4.45 \mathrm{~mm}$, respectively, which are 99.56 and 99.63 percent accurate.

Results show the effectiveness of this procedure in calculating the location of the bases of sprung masses in two dimensions. The basis of this research contributes to the determination of bone position over time that will increase the potential of understanding fundamental, rigid body and joint motions in a clinical setting using noninvasive methods. 


\section{Chapter 1. Introduction}

\subsection{Motivation}

Since the early days of man, bodies have been in motion. These motions are due primarily to the central nervous system and the signals it sends to the motor neurons of the muscles. These signals cause the selected muscles in the muscular system to have a voltage potential, which in turn activates the muscles. As these muscles activate, they contract and the skeletal system moves.

The analysis of an animal's motion can potentially reveal whether or not abnormalities are present within the subject and aid in the diagnosis of an abnormal condition. Several common examples are arthritis, broken bones, and lameness. Such conditions can create pain, reduce the range of motion, and cause the subject to guard against using the afflicted body part and compensate, for example, by limping. It is desirable to recognize the symptoms leading to these afflictions prior to compensation being necessary so that treatment is kept to a minimum in both time and expense.

The effectiveness of medical procedures could benefit from motion analysis as well. Many treatments performed today are attempts at improving the quality of life for an individual. As an example, painful bone spurs are a product of osteoarthritis and can limit the motion of a joint. Once the spurs are removed, whether through surgery or medication, mobility will be restored to the joint and the pre- and post-treatment differences can be evaluated.

Prosthetic design heavily relies on data obtained from motion analysis. Once a body part is missing, whether from birth or due to injury, an artificial replacement can be attached to partially restore the functions that were lost. To create a practical and useful prosthesis, the range of motion and capacity of the body part it replaces must be taken into account and replicated. 
Sports require rigorous effort, both mental and physical, in a competitive activity. As the level or degree of experience increases, basic motions are mastered and the finer points of actions require more attention. Motion analysis can be used to compare one's current motion to one that is desired for increasing any combination of efficiency, speed, or strength among others. Alternatively, the motion can be dissected at varying time steps to provide instruction for incremental changes in motion.

\subsection{Motion Analysis}

This research was conducted intending to further the application of MEMS (micro electromechanical systems) accelerometers as a part of surface-mount sensors for resolving underlying skeletal motion. Surface mounted markers are the most commonly used means of analyzing bodily motion and can be used in both science and medicine. These markers are tracked over time and quantitatively portray how the body moves during a given activity.

Many systems commonly used today to measure the displacement of the body over time, but do not accurately track the motion of the fundamental, rigid bodies of the skeleton. These systems rely on markers typically located on the surface of soft tissue and, given any motion, the tissue will have a motion relative to that of the underlying bone. Soft tissues, including muscle, should not wholly define the motion undertaken by a body. They should only be considered as the motors enabling the motion of the frame. Superficial tissues provide the majority of shape differences in bodies, but the arrangement of the skeletal system remains relatively unchanged throughout the population and should be defined as the standard to which motion analysis is to be measured.

To measure bone motion accurately, methods that rigidly fix markers to the bones [Fuller, et al., 1997; Murphy, 1990; Taylor, et al., 2005] have been used. However, invasive measures 
can be tedious and painful and are not acceptable as clinical practice. Therefore, being able to measure the bone accurately using non-invasive methods is a highly desired objective.

\subsection{Objectives}

The first objective of this thesis research was to design and fabricate an experimental apparatus that represents the major components of a limb common in animals. This apparatus was to simulate the properties and motions of the body segment in two dimensions.

The second objective of this thesis research was to collect acceleration data on sprung masses from accelerometers on the experimental apparatus. These accelerations attempt to model those present on the surface of the soft tissue along the two axes in which the majority of motion occurs. The reasoning behind the need for explicit acceleration data in dynamic studies is the fact that when position, or any, data are differentiated, noise is introduced and can contaminate the results. For increased accuracy in the analysis, Codamotion ${ }^{\mathrm{TM}}$ (Charnwood Dynamics Ltd., Leicestershire, United Kingdom) sensors, acquiring position data, were used both on the sprung mass with the accelerometer and on the rigid base. Weights were applied to both sprung sensor sets determining their influence on the results.

The third objective was to analyze the collected acceleration data to determine the distance separating the soft tissue and underlying bone. This distance allowed for the calculation of the bone position at each particular location. This calculated bone position was then

compared to the actual position determined by Cartesian Opto-electronic Dynamic Anthropometer (CODA) markers mounted on the skeletal model. This method will help validate future work attempting to calculate underlying bone motion with only accelerometers or similar measurement devices attached to the surface of the skin. 


\subsection{Thesis Outline}

The diagnosis of many common ailments traditionally relies on the observational expertise of clinicians. However, the severity of which these ailments are afflicting the patients is more difficult to agree upon between clinicians due to the subjective nature of diagnoses. Kinematic gait analysis is one approach to accurately find abnormalities in a subject's motion. This analysis can help solve the questions of when, where, and how the ailments affect the motion of a body.

This work is presented in six chapters: Introduction, Background, Modeling the Dynamic Motion of Soft Tissue, Design of the Test Station, Experimental Results and Analysis, and Conclusions and Recommendations. The background of this field is discussed in Chapter 2. Biomechanics, including brief anatomy descriptions, is presented in Section 2.2. Gait analysis is covered in Section 2.3, which includes a discussion of methods used from the initial stages during the latter part of the $19^{\text {th }}$ century to those investigated in recent years. The chapter concludes with the analysis of soft tissue motion relative to the bone and steps taken to reduce its influence on data.

The methods and modeling used in this research are covered in Chapter 3. This chapter is devoted to the mathematical strategies applied to solve the proposed problem.

The design and construction of the experimental apparatus is covered in Chapter 4. This mechanism allows for the extensive range of motion encountered in a body during high levels of activity to be experienced by a representative double pendulum.

Experimental results are discussed in Chapter 5. In this chapter, the comparison will be made between the bone positions calculated from soft tissue data and those from direct measurement on the bone itself on the designed and fabricated test stand. From these results, the effectiveness of the proposed method is determined. 
Conclusions of this research are presented in Chapter 6. Suggestions for future ventures regarding noninvasive procedures are also covered in this chapter. 


\section{Chapter 2. Background}

\subsection{Introduction}

Biomechanics is defined as the study of the mechanical laws relating to the movement or structure of living organisms [OED, 2006] and is the basis for gait analysis studies. More importantly, to measure the motion of the rigid bodies comprising organisms is the goal in these studies. This chapter presents information regarding the anatomy of subjects and the measurement and error-reduction methods used in previous studies attempting to accurately resolve rigid body motion in animals and humans.

\subsection{Biomechanics}

Mechanics, the science which describes and predicts the conditions of rest or motion of bodies under the action of forces [Beer and Johnson, 1996], is comprised of three parts: the mechanics of fluids, rigid bodies and deformable bodies. The latter two of these parts are under investigation in this research and both contribute to the study of dynamics, the part of mechanics that deals with bodies in motion.

Stemming from dynamics is another approach to the study of bodily motion. Kinematics is the study of position and its time derivatives without consideration of external forces and

moments. Specifically, it deals with the positions, velocities, and accelerations of points and with the angular positions, angular velocities, and angular accelerations of solid bodies [Waldron and Kinzel, 1999].

The study of biomechanics is a direct application of the mechanical laws to living organisms. The mechanical body structures of these organisms are presented in the following sections. 


\subsubsection{Bones}

\subsubsection{Function}

Bones provide the structural base for body segments and are generally modeled as rigid links. They provide several necessary functions; including support for the body and its associated tissues, protection of internal organs, and a linkage system that provides the basis of force transmission from one link to another [Fuller, 2001].

\subsubsection{Anatomy}

Two types of bone tissue are used to construct these links, cortical and cancellous. Cortical bone is dense and is located along the bone shaft and the comprises the shell on the surface while cancellous bone is less dense and lies in the interior and is arranged in a lattice-like structure. This lattice of cancellous bone fibers, made of dense and fibrous tissues called trabeculae, aligns itself to the applied exterior forces to better distribute the load throughout the entire structure. The open spaces within the lattice also provide a suitable location for the production of blood cells and storage of minerals. The ratio of these two tissues varies between bones, as it depends on the size and functional characteristics of each bone [Gray, 1995].

Bone, being a living tissue, is constantly broken down and regenerated depending on how it is being used and what nutrients the body needs, the most abundant in the bone being calcium. When a particular body part undergoes increased levels of stress, the bones in the affected area will generally respond by strengthening. The two types of cells responsible for these actions are osteoclasts and osteoblasts, which assemble in groups known as basic multicellular units (BMU) [Nigg and Herzog, 1999]. Osteoclasts remove the unneeded cells and osteoblasts regenerate cells in the needed locations. However, the two operations do not occur at the same rate and the BMU-remodeling system does not operate at a constant frequency over time. As the body ages, 
this frequency of remodeling increases and, accompanying the imbalances of bone resorption and formation, the geometry and composition of bone is negatively affected and the bone is weakened [Nigg and Herzog, 1999].

\subsubsection{Joints}

\subsubsection{Function}

Joints are located at the contact points between bones. Three classifications are used to categorize joints, with two allowing motion between the bones when necessary. There are synarthrodial, amphiarthrodial, and diarthrodial joints. Synarthrodial joints, with prefix "syn" meaning "together", connects bones with tough fibrous tissue creating immovable joints. These create the cranial sutures connecting bone plates that create the skull. Amphiarthodial joints allow only slight movements and include the invertebral discs, separated by fibrocartilage, and the proximal ends of the tibia and fibula in the lower legs. Finally, the joints allowing maximal motions, also known as diarthrodial joints, come in many varieties depending on the type of movement being undertaken. They range from a simple hinge joint, which permits motion through only one axis of rotation, such as an elbow, to a ball and socket joint, which allows multiple degrees of freedom, such as the hip connecting the acetabulum and femur.

\subsubsection{Tendons and Ligaments}

To link two or more bones at a joint requires flexible members able to stabilize the joint and withstand forces attempting to separate the rigid bodies. These members, made of fibrous tissues in parallel and slanting arrangements, are known as ligaments. Similar elements having a parallel structure of fibrous tissues are tendons, which connect muscle and bone [Gray, 1995]. Tendons transmit the forces generated by the muscles to the bone. Two components of this force are borne by the joint and ligaments, which are the rotary and, depending on joint angle, the 
stabilizing or dislocating components. The former causes rotation of one bone relative to another about the joint and the latter pulls the connected bones together or attempts to pull them apart, respectively.

\subsubsection{Contact Surface}

Articular cartilage is located at the contact surfaces in high-load joints. The four basic functions of this tissue are to lubricate, resist wear, absorb shock, and limit the load bearing duties [Fung, 1993]. Lubrication is due to synovial fluid that seeps out of pores when load is applied. This seepage also contributes to the shock absorption abilities of the joint, as the faster a load is applied to the joint, the greater the resistance of the fluid exiting the porous membrane. This also works with slower applications of load, which allow greater seepage of fluid from the tissue. Synovial fluid exhibits very low friction coefficients, between $0.0026-0.0038$ in a test specimen joint, which contributes to superior wear resistance [Fung, 1993]. However, over time,

ragged projections can form on the articular surfaces due to wear and tear. Even a change in synovial fluid viscosity can cause erosion to occur and limit the functionality of the joint [Fung, 1993].

\subsection{Gait Analysis}

\subsubsection{Definition of Gait Analysis}

Gait analysis is an area within biomechanics that quantifies the motion of an animal. There are two categories of measurements that are used for analyzing the gait of any organism: qualitative and quantitative. The former is a relatively quick, subjective analysis based on observation, and the latter is a recorded set of data that are used to discover and diagnose motion with a much higher degree of accuracy. 


\subsubsection{Qualitative}

Qualitative measurements have been widely used in sports based on display, such as the scores awarded by the judges in equestrian events [Clayton, et al., 2001], which can be likened to the types of judging present in many other presentation sports. Frequently, posted results show that not all judges award the same score value. This subjective measurement practice, however, is not limited to sport. Clinically, this is commonly practiced in the physician's office and the subjective analysis is used to diagnose common abnormalities in gait, such as rheumatoid arthritis in humans, lameness in horses, or hip dysplasia in dogs. In equines, the different ways the body can react are more limited than the range of possible ailments. Only a few disorders exhibit irregularities in gait specific to that particular disorder [van Weeren, 2002]. These assessments of subtle irregularities in gait and their interpretation are often delicate or complex, in which case the diagnosis strongly depends on the expertise of the orthopaedic surgeon [Weishaupt, et al., 2002].

Bodily reactions are not the only difficulties present at the time of analysis that can cause confusion in an examination. Physiology is a second complication since no animal is perfectly symmetrical. These biological variations within the animal and species are large and can produce a gray area when attempting to discern pathological and physiological motions [van Weeren, 2002].

Again, different examiners can come to different conclusions, which is why many people opt for a second opinion based on their analyses. These difficulties further the opinion that qualitative measurements provide relatively quick, but highly debatable results.

\subsubsection{Quantitative}

By introducing quantitative gait assessment, additional assistance can be provided to the subjective measurements taken by clinical experts when determining faint asymmetries of gait. 
Using numerical data, repeatable analysis can be performed on subjects and can be extremely useful for patient monitoring and evaluating treatment modalities [van Weeren, 2002]. The pioneering development of gait analysis systems has altered the perception of equine gait [van Weeren, 2002], all of which began in the latter part of the $19^{\text {th }}$ century.

\subsubsection{Preliminary Methods}

For thousands of years, man was only able to formulate questions concerning the movements of animals even though characterizations have appeared scrawled on the walls in ancient caves. In ancient Greece, the great philosopher, Aristotle, was drawn to the subject of animal locomotion and dedicated much of his attention towards the functions of their limbs during locomotion. The methods involved in these studies, however, were never recorded for later investigators [Muybridge, 1899].

Several attempts to investigate animal locomotion were successful in the later part of the $17^{\text {th }}$ century, but none of them were able to record pictures of actual animal motion. It was not

until the middle of the $19^{\text {th }}$ century that picture taking was advanced enough to photograph objects in motion by using the collodion process, which involved light sensitive plates. Thus was the process used when the question of whether "the possibility of a horse, while trotting even at the height of his speed - having all four of his feet, at any portion of his stride, simultaneously free from contact with the ground" was investigated [Muybridge, 1899] and interest in gait analysis was spurred.

In the spring of 1872 at a racetrack in Sacramento, California, Eadweard Muybridge [1899] conducted his famous experiment that captured in time the silhouette of the racehorse Occident with all four hooves off the ground. Satisfied with the confirmation of this theory, he began to investigate more animal motions with the help of Leland Stanford at his stock farm, and 
later, with more comprehensive work, at the University of Pennsylvania. Through his research, he developed the zoöpraxiscope to display still images in rapid succession. Thomas Edison, arguably, improved upon this by implementing the work of Etienne-Jules Marey and his celluloid ribbon by inventing the kinetoscope, which could show short clips of animation from moving figures [Muybridge, 1899].

In order to obtain successive positions of figures in motion prior to the availability of a video camera, Muybridge [1899] needed to use multiple cameras and have each record one image over time. The studio had a 37-meter long lateral background; the figure in motion would move along with thin lines, both horizontal and vertical, spaced $5 \mathrm{~cm}$ apart and thick lines with $50 \mathrm{~cm}$ spacing for use when the photographs were developed. Aligned parallel to the background at a distance of $15 \mathrm{~m}$ was a battery of cameras, comprised of a maximum 24 cameras. Positioned behind the figure in motion in vertical fashion was a battery of 12 cameras to photograph "rear foreshortenings" and arranged forward of the figure, horizontally, and $60^{\circ}$ to the background, was one final battery of 12 cameras to capture "front-foreshortenings". Located at camera 1 of the original battery, the operator could view the chronograph and operate the motor for the electric circuits responsible for the successive exposure of cameras when the object in motion is in the line of sight of each. The first camera in each battery was exposed manually and simultaneously, which proceeded in succession through all cameras in each battery based on a pre-defined time interval set by the operator [Muybridge, 1899].

Determining the time between each image was done by a chronograph using a tuning fork vibrating at $100 \mathrm{~Hz}$ and smoke-blackened paper. The vibrations of the tuning fork would be recorded on the paper with a certain mark and as the exposures of the cameras occurred, another different mark would be made. Therefore, the time spanning successive photographs could be 
recorded. For greater accuracy when analyzing faster motions, the spaces between the fork's marks were divided into ten equal portions creating measurements that could be made on the order of thousandths of a second [Muybridge, 1899].

Basic motions of animals can be analyzed from the pictures taken by Muybridge [1899] using the grid provided on the backgrounds, but the perspective of the camera and body can limit the accuracy of the results. Using sequential cameras, it was difficult to record images where the body in motion remained in the same relative position to cameras. Shifting to either side of the lens or altering the distance from the camera affected how effective the grid was for quantifying the motion.

\subsubsection{Photogrammetry}

\subsection{Definition of Photogrammetry}

Muybridge's [1899] work with motion analysis involving photographs laid the foundation for the technique of photogrammetry. "Photogrammetry is the science and art of making precise and reliable measurements from images" [Gruen, 1997]. These images can come from many available sources and are not limited to photographic illustrations. The goal of this method is to determine the three-dimensional coordinates of an object relative to a pre-defined coordinate system. This is accomplished by triangulation using the two-dimensional images obtained by two or more cameras. This technique is commonly used to analyze the movement of an object. Movement analysis aims at gathering quantitative information about the mechanics of a system during the execution of a motor task [Cappozzo, et al., 2005].

\subsection{Camera and Marker}

Cameras must be able to distinguish what portions of an object are under analysis by using well-placed markers easily identifiable to the camera. For any three-dimensional analysis, 
there must be a minimum of two cameras concentrated on viewing the object in motion. Through triangulation algorithms, the location of each marker is located in space and relative to a coordinate system already defined in the laboratory.

There are two categories of markers used in this process, passive and active. Passive markers are standalone balls with a retroreflective coating that incorporate the use of an external light source. Spheres are required so that, from any camera at any time instant, the marker's shape remains unchanged and appears circular in a two-dimensional projection. In most cases, the light source is a grouping of light emitting diodes (LED) that lies on the perimeter of each camera and pulses for each instant that particular camera records an image.

Active markers, also known as powered markers, incorporate electrical components necessary to be located by the cameras and do not necessarily need to be spherical in shape. Each marker pulses light in sequence that can be seen by the cameras when images are being recorded.

Regardless of what category a marker falls into, there is an inherent difficulty associated with marker occlusion. To compensate, one of three common solutions is used. The computer system interpolates each marker position based on data collected before and after its occlusion, redundant markers are placed in the immediate area, or there must be additional of cameras that can view each marker in times where others cannot. In addition, there must always be at least two cameras viewing each marker to ensure proper three-dimensional locating of the marker in space.

There are many methods available to attach markers to a subject and they fall into two distinct categories, invasive and noninvasive. Invasive measures are generally assumed to require minor surgery or cause physical distress for the subject whereas noninvasive measures are more often simple and pain-free. 
Examples of attaching noninvasive markers to subjects can be the use of adhesives [Fuller, et al., 1997; Clayton, et al., 2001] and bands or straps [Cappozzo, et al., 1996; Fuller, et al., 1997; Lucchetti, et al., 1998]. Obviously, these are relatively harmless and can be attached and removed quickly and easily. For invasive markers, the most common approach is the use of pins or screws inserted through the soft tissue and attached rigidly to the bone [Lafortune, et al., 1992; Fuller, et al., 1997; Taylor, et al., 2005], which then can have markers mounted on the free ends. Another approach is with the use of an external fixator. An Ilizarov device is a cage-like, modular apparatus composed of rings and rods that slips over the limb under analysis and anchors to the bone with pins [Alexander and Andriacchi, 2000]. A third approach is with an external fracture fixator that attaches to broken bones at the healthy ends with screws and helps align the bone for proper healing as well as support [Cappello, et al., 1997]. Both devices, once mounted, allow for a sturdy foundation onto which markers can be attached to the subject. However, due to moral, ethical and other practical limitations, invasive measures are not used to the extent of its alternative. The applications of these methods are further discussed in the later section regarding soft tissue artifacts.

\subsection{Radiography}

Using images produced by radiographs allows for the direct determination of the underlying bone position, more so with the insertion of radiopaque markers. However, this form of image capture is invasive in two ways. First, the markers must be surgically attached to the bone, and second, the subject being analyzed is exposed to radiation from X-rays during exposure over the desired analysis period [Tashman and Anderst, 2003], the levels of which can be potentially dangerous over an extended study.

The clarity of resulting images depends not only on the thickness of tissue in the area and the absorption of it along with the bone, but also on the exposure speed. Like a still-image 
camera, a radiograph camera has an exposure time, and during dynamic situations the target object can appear blurred. Therefore, high-speed cameras must be used for increased image quality.

A study was performed to estimate motion of a canine knee using high frame-rate biplane video radiography and computed tomography (CT) images [Tashman and Anderst, 2003]. The goal of this work was to demonstrate the precision that can be attained in motion analysis when using an alternative technique on a group of five dogs in a controlled dynamic motion. Biocompatible tantalum markers were inserted near the knee joint on both the femur and tibia from which orthogonal coordinate systems were defined. Using CT scans, three-dimensional bone models were created and anatomical landmarks were identified and used to create the anatomical coordinate system. Bone-embedded radiopaque markers were also identified, helping to create the marker coordinate system, and its orientation was related to the anatomical system using standard transformations [Tashman and Anderst, 2003].

The measure of the precision of this technique was dependent on the standard deviations of known inter-marker distances when compared to calculated frame-captured distances. The sizes and shapes of the legs in this group of dogs were not constant so marker placement distance for each varied. For inter-marker distances, the average standard deviation across the group was $0.064 \mathrm{~mm}$ when measured with an intact ACL and $0.072 \mathrm{~mm}$ after surgical transection of the ligament. The actual spacing was between 5.5 and $31.5 \mathrm{~mm}$. Using estimates for ACL origin and insertion points, standard deviations involved with tibio-femoral translation averaged 0.14 mm across all three directions over several millimeters of travel [Tashman and Anderst, 2003].

The use of this radiograph technique demonstrates the high level of precision possible during in vivo motion studies that is not available in the more common forms of motion capture. 
Though it provides accurate values, there are several limitations that prevent it from being widely applicable in the clinical environment. For traditional motion analysis in multiple subject and trial studies, the cost involved with surgical procedures and radiation exposure may not be suitable. Though the authors claim a radiation dosage of only $200-300 \mathrm{mR}$ per trial in this study, not a high level according to radiological standards, only 0.5 to 1 second of exposure time was used per trial [Tashman and Anderst, 2003]. This drawback can severely limit comprehensive gait studies or any that require a greater length of analysis.

\subsection{Magnetic Resonance Imaging}

Magnetic Resonance Imaging (MRI) is a fairly new tool being evaluated for conduct of kinematic analyses on the body. Using strong magnetic fields and non ionizing radiation, this noninvasive approach allows for the recognition of bone and tissue within the body in multiple dimensions. This visualization method allows the user the opportunity to locate specific points on the skeletal body over time using sequential captured frames.

The inter- and intraobserver reliability of manually locating particular anatomical landmarks over a defined motion of a body using MR images and a motion predicting algorithm has been studied [Lerner, et al., 2003]. Nine pre-defined anatomical points on the distal femur and proximal tibia were located in six sequential images of a subject's knee while lying in the prone position in the scanner. The range of motion spanned from a full extension reference to near the limit of the space available in the gantry at $19^{\circ}$. For the study, an algorithm was created to use the digitized reference points located in full extension, holding their relative locations on the segment steady during knee flexion, and predict their positions in ensuing MR images. Comparisons between these predictions and those from manually locating the points were made. The average measurement errors were $\pm 0.51 \mathrm{~mm}$ for inter-observer and $\pm 0.41 \mathrm{~mm}$ for intra- 
observer with a maximum of $\pm 3 \mathrm{~mm}$. The authors concluded that even though the accuracy provided by manually locating these points was good, in motion tracking situations the algorithm provided a more realistic representation of knee kinematics and processes the data more quickly than observers.

An intertrial reliability study was later performed on a group of subjects executing a flexion/extension exercise similar to that used by Lerner, et al., [Barrance, et al., 2005]. Predefined locations for anatomical landmarks on the femur and tibia were located on MRI scans and tracked over the designated motion in two sessions for five individuals. These individuals were to undergo ACL surgery, but the leg under analysis was their healthy one. The root mean squared (RMS) differences of tracked anterior/posterior movements of the tibia between the two sessions had an overall value of $1.44 \mathrm{~mm}$ with a maximum of $4.62 \mathrm{~mm}$. Tibial rotation was found to have an overall RMS difference of 2.35 degrees with a maximum of 7.99 degrees. These values, according to the authors, may not be completely accurate because, between the first and final trials, the kinematics of the knee may have been altered for any variety of reasons relating to post-surgical experiences.

Several limitations of MRI methods hinder the use in broad studies for motion analysis. A significant limitation is the restricted size of the environment able to be analyzed [Tashman and Anderst, 2003; Barrance, et al., 2005]. Equal in restriction of motion studies are the low frame rates available with the MRI systems [Tashman and Anderst, 2003]. However, there are several advantages that make this method attractive. The noninvasive nature allows for easier implementation and greater convenience for repeated trials or larger groups of subjects during studies. 


\subsection{Photogrammetric Errors}

As with any measurement process, errors are introduced that can skew the output of the system. These errors fall into two categories: instrument and soft tissue artifacts [Chiari, et al., 2005; Cappozzo, et al., 1996; Lucchetti, et al., 1998].

There are two types of instrument errors, systematic and random. Systematic errors come from calibration inaccuracies while random errors come from electrical noise, the digitization process or marker shape image distortion [Chiari, et al., 2005]. Both errors lie within the instrumentation, most often the camera itself. These can result from such abnormalities as improper lens shape, misalignment of lens components, prism distortion from improper assembly, and other electronic errors [Chiari, et al., 2005; Gruen, 1997].

Soft tissue artifacts come as a result of the skin surface moving relative to the underlying bone. This error shall be explained further in a later section. In the same section, the locations for markers in order to minimize the effect of such errors prior to the application of reduction methods will be discussed.

\subsubsection{Sensor-Based}

Several means of acquiring motion data are available that do not rely on the camera and marker methods described previously. This is why another type of data acquisition is to be considered, referred to by this research as sensor-based, since it breaks away from the commonly used system approaches to provide a new approach to data collection from motion studies using various types of sensors. The first group of data collectors under consideration uses the technology provided by microelectromechanical systems (MEMS). Specifically, gyroscopes and accelerometers have been used in recent years to analyze motion. 


\subsection{Accelerometer}

Most accelerometers being produced today use the same principles in their designs, which suspend a small mass by springs within housing. As the housing is moved, the internal mass displaces and the length of the spring changes. An electrical signal produced by the sensor changes with respect to the spring length change and is proportional to the acceleration being experienced in the sensitive direction.

A study performed by Lafortune was done to measure the acceleration at a point along the tibia of a subject [Lafortune, et al., 1991]. In his study, he rigidly mounted a tri-axial accelerometer to the proximal end of a subject's tibia using a Steinmann pin. Acceleration data were acquired during a walk, with and without shoes, and runs at two different speeds. No position data was calculated following the analysis.

The results of using this sensor demonstrate how accurately the values of acceleration are replicated over successive cycles of a given gait. They also produced plots that confirm anticipated behaviors of accelerations of different conditions. For example, a general assumption is that the acceleration values with a shoe, which provides a layer of cushion between the foot and contact surface of the treadmill, on will be less than those produced from a barefoot approach. Another assumption was that larger acceleration values would be recorded when traveling at higher velocities due to the speeds at which the lower limbs contact the treadmill surface. Both hypotheses were confirmed through this study. Lafortune [1991] subsequently exhibited the applicability and reliability of using these sensors for future motion studies.

\subsection{Gyroscopes}

There are several different designs available when considering gyroscopes; spinning rotors, laser, and vibrating mass, but only one is actually suitable for use in motion analysis. The 
vibrating mass, because of its compact size and low power requirements, is better suited than the other two [Luinge, 2002]. These sensors are used to acquire angular velocity measurements and rely on Coriolis forces to do so. Inside the housing is a mass that is put into a given motion along an axis and is able to slide along another perpendicular to the first. When the sensor experiences an angular velocity, a Coriolis force is exerted on the mass. From this force and knowing the mass and velocity of the mass at that instant, the angular velocity of the sensor can be determined [Luinge, 2002]. A well-known limitation of the gyroscope is an integrationinduced error commonly known as drift that skews the output over time, due mainly to offset fluctuations and measurement noise [Luinge, 2002]. In motion analysis, the gyroscope is commonly partnered with an accelerometer.

\subsection{Combination}

A common practice in motion tracking using sensors is the combination of both accelerometers and gyroscopes. Used together, there is a source for both acceleration and angular velocity measurements, which give information that can be used to calculate actual positions of points at any instant in time. This was the approach used by Sakaguchi, et al., to determine real-time motion of a person's arm [Sakaguchi, et al., 1996].

In this study, the data gathered from both sensors, total acceleration and angular velocity, were applied to the equation for acceleration (including rotational, centrifugal, Coriolis, and gravitational components) which was solved to yield all position and motion information. Two models were used that showed the effectiveness of this method; one was a rotating bar with one sensor pair and the other was a simulated human arm with two pair, one on each segment. Error between actual and calculated values decreased with the combination as compared to data from each sensor individually. 


\subsection{Magnetic Tracking}

Magnets have been used to determine positions over time by sensing magnetic fields. A transmitter emits magnetic fields that are collected by a receiver which has its position and orientation measured as it moves.

A study performed by Karduna, et al., used this method to measure the three-dimensional kinematics of a human scapula and did so with two mounting methods [Karduna, et al., 2001]. The acromial method attached the sensors directly onto the skin with double-sided tape and the tracker method fastened the receiver to a mechanical platform that was attached to the skin with adhesive Velcro strips. An additional tracker was attached to pins rigidly fixed to the scapula for comparison to skin-mounted results.

In motion, both of these skin-based methods demonstrated the same general movement with reasonable accuracy as that of the bone-based method. The differences between the bone and skin data were assumed to be purely soft tissue motion. One of the addressed criticisms, similar to many other skin-based analyses, was that this method may only be usable on lean subjects with low levels of soft tissue covering the bone due to the greater amount of erroneous motion available with greater coverage. The size and bulk of sensors of this type may also contribute to greater skin travel.

\subsubsection{Soft Tissue Artifact}

\subsubsection{Definition of Artifact}

As mentioned previously, errors introduced to the system fall into two categories: instrumental and soft tissue artifacts. The former has already been described and this section aims to explain what soft tissue artifacts are, how they affect the quality and accuracy of motion data, and what methods have been developed to reduce these errors. 
Soft tissue artifact is defined as an error that "originates at the interface between the measurement instrument and the substrate which is the object of the measurement" [Cappozzo, et al., 1996]. In simpler terms, Cappozzo, et al., also state that it is the relative movement between the marker on the surface of the soft tissue and the underlying bone. Two categories exist that comprise this error: deformation within the marker cluster and rigid cluster movement relative to the bone [Lucchetti, et al., 1998; Taylor, et al., 2005]. Both of these categories are addressed in the following sections as methods designed to reduce their influence are described.

\subsubsection{Artifact Influence}

Soft tissue artifact is believed to be the largest invalidating source of experimental error [Stagni, et al., 2003; Cappello, et al., 1997] and is the largest limiting factor of systems based on the use of skin markers [Andriacchi, et al., 1998]. Unfortunately, this error is also the most difficult to eliminate due to several factors, including it being subject and task dependent [Fuller, et al., 1997]. The most comprehensive reason was noted by Fuller, et al., when comparing the frequency content of both skin- and pin-mounted markers. Both markers share the same frequency domain, although at significantly different power levels, leading to the conclusion that smoothing techniques are not applicable for the removal of artifacts and that surface markers are inappropriate when attempting to portray motion of the bone accurately [Fuller, et al., 1997].

Fuller, et al., did not perform any reduction methods to their skin marker data to compare directly to the pin data, but they did produce documentation regarding the discrepancies between the two sets that show how profound soft tissue errors are.

In an experiment involving both pin-mounted arrays of markers and individually skinmounted markers located on the lower extremity, the subject performed four tasks, two of which were analyzed for soft-tissue motion. These two were stationary bicycle and normal gait. For 
both, the distances from the center of the pin array to each skin marker were determined in a neutral position and were compared to distances calculated during motion in three dimensions. The differences determine the soft-tissue motion. Two skin markers, one near the pin-mounted array at the greater trochanter with a thick layer of tissue between it and the bone, and one near the knee with a thin layer of tissue, were presented. For both, the tissue displacement relative to the greater trochanter and femoral condyle, respectively, reached upwards of $20 \mathrm{~mm}$ in the bicycle trial and the same value was measured for the latter marker in the gait trial.

\subsubsection{Artifact Reduction Methods}

The severity to which the soft tissue artifact skews the data from skin-mounted markers is quite evident now. Numerous methods have been developed to reduce the influence of this error, but none has succeeded in eliminating it altogether. Each of the following methods approaches this problem in different ways, but as will be shown, each has its own benefits and limitations.

\subsection{Double Calibration}

The basis of the double calibration method lies in the manner which anatomical landmarks are located on the subject to relate to skin marker clusters. Using the calibrated anatomical systems technique (CAST), considered a base for standardization for definition of anatomical landmarks and their calibration [Cappozzo, et al., 1995], the three-dimensional positions of landmarks are defined at two instants during the performance of a given task relative to the marker cluster [Cappello, et al., 1997]. For the referenced study, the extremes of hip and knee motion during cycling, full flexion and full extension, provide the instances for calibration (reference configurations) which takes into account not only the rigid motion of the cluster relative to the bone, but also the deformation of the cluster due to inter-marker movement.

When all marker positions were collected at the two instants, one configuration was overlaid on the other with coinciding cluster centroids that showed marker position variation 
between the two. A time-varying model and associated calibration parameters were then defined under the assumption that the position variations between the configurations were linear and can be defined through linear interpolation. During the motion study, the calculated model and landmarks are then compared to the actual cluster of markers and landmarks recorded at that instant where transformations are estimated between them [Cappello, et al., 1997].

Through validation using an external fixator assumed to be rigidly attached to the bone, this method proved to be simple and very effective at reducing the error associated with the location of landmarks and bone position. However, several assumptions allowing for ease of use also contributed to the limitations of this method. The first was the assumption of linear position variation of the markers within the cluster and with respect to the landmarks from one calibration posture to the other. This was further complicated by a second assumption that the artifact contributions from muscle contractions and inertial effects were minimal. These effects in dynamic situations could possibly further complicate the validity of the first assumption. Further analysis for this assumption was suggested to reduce negative kinematic effects. Finally, taking static calibration measurements at the defined postures for an investigation employing a dynamic event can only lead to more inaccuracies in the results when considering the previous statement and the effect it has on distances measured between points. The investigators did address the idea of additional calibration postures, which would aid in the interpolation and instantaneous position values, but it would also complicate a simple and rather effective procedure [Cappello, et al., 1997].

\subsection{Point Cluster Technique}

The point cluster technique (PCT) was designed to determine the artifact associated with the movement of the non-rigid markers in the cluster placed on the skin surface for in vivo 
applications. The name originates from the way in which markers are placed on the skin; a cluster made of no less than three points distributed uniformly in three dimensions about the skin surface [Andriacchi, et al., 1998].

To reduce the artifact error, this technique assigned arbitrary mass values to each point. For each time step, the center of mass for this cluster and its eigenvalues and vectors are calculated. The trick lies in the calculated eigenvalues because, if the skin is acting like a rigid body, then these values remain unchanged from the recorded static reference frame. However, if a change has taken place, there is non-rigid movement present and the values will deviate from the reference. Therefore, varying the masses for each point to minimize the eigenvalue changes in each step can reduce the perceived influence of the artifact [Andriacchi, et al., 1998]. These masses have significant influence towards the calculation results. If a point moves relative to the cluster as a whole then its effective mass is decreased, whereas if there is no relative movement then its mass is increased.

Andriacchi, et al., performed their analysis as a comparison to one done by Lafortune et al. [1992], which made use of intra-cortical pins to demonstrate the usefulness of their noninvasive technique. The analysis showed how the two approaches produced comparable results. In knee rotation, flexion data was practically identical, whereas for abduction/adduction and internal/external rotations, the plots do not match and only the patterns appear similar. The same can be said for the translational displacement of the femur with respect to the tibia in three directions [Andriacchi, et al., 1998]. Though eigenvalue changes were noted, which signified to what degree non-rigid motions were taking place, the effectiveness of this technique is debatable based simply on the fact that a direct comparison was not made with pins in the same experiment with the same subjects. 
Limitations to this method were noted by the authors. The first concerned the need of an abundance of markers over the surface of the limb under investigation and the ability for each of these to be monitored without difficulty. This problem can possibly be alleviated with the use of powered markers having the ability to be tracked by a computer. The last concern was that of marker position to ensure there was no symmetry in the clusters [Andriacchi, et al., 1998]. Further analysis was conducted on the applicability of this method as the next two methods produced a direct comparison of it with new techniques.

\subsection{Optimal Common Shape Technique}

The optimal common shape technique (OCST) is one that attempts to reduce marker movement influences by optimally configuring the skin markers throughout the set of measured timeframes. It addresses the issue of individual marker fluctuations, but does not attempt to account for the rigid movement of marker cluster as a whole relative to the bone [Taylor, et al., 2005].

The procedure for determining the optimal shape of marker clusters over the entire time of measurement partially using Procrustes methods, which are recognized in statistical shape analyses [Taylor, et al., 2005]. Taylor et al., however, modified the approach to include a constraint that the distance between markers in each set remains equal and constant. Taking into account all of the configurations for each marker set over the course of measurement, the optimal configuration for each set is determined. In doing so, the relative movements of markers within the cluster are removed and therefore the accuracy of rigid body estimation is improved [Taylor, et al., 2005].

Taylor, et al., conducted their study to examine the reduction effectiveness of their newly developed method to that of Andriacchi, et al. [1998]. The two were used to estimate the 
proximal joint centers of the femur, tibia, and metatarsus. The averaging of raw data itself was analyzed for another comparison and all data was validated with markers on pins mounted directly to the bones.

Through the presentation of the data in this study, it becomes evident how effective each of the three methods was at reducing the effect of marker motion in joint center estimation. The authors correctly hypothesized the relationship of soft-tissue volume and marker error stating that an increase in tissue results in an increase in error. This is seen in the average position error plots regarding the three joints with the femur having the greatest error, followed by the tibia and metatarsus. In comparing the different methods, all three seem suitable for areas with large soft tissue coverage, but the PCT shows increasing errors as the amount of tissue decreases. The other two, OCST and raw averaging, show similar plots revealing that even the OCST may not be a significant improvement of joint position estimation. When reducing the average error down to three anatomical directions, medial-lateral, posterior-anterior, and distal-proximal, the same pattern remains. The latter two methods show nearly identical error values for the lower two joints.

Since this study focused mainly on the validity of the OCST, the requirements for the PCT were not a priority and may have led to inaccurate results, namely the lack of sufficient markers and less than optimal distribution along the limbs [Taylor, et al., 2005]. Regardless of preparation, the study shows the shortcomings of the methods when compared to the raw data results. Along with neither being a sufficient improvement, neither addresses the motion of the cluster itself to the underlying bone, which is, as already mentioned, the greatest source of error in artifact composition. 


\subsection{Interval Deformation Technique}

Following the development of the PCT, the authors expanded on this method and created the interval deformation technique (IDT) [Alexander and Andriacchi, 2000]. Where the PCT lacks, the IDT builds upon by including the correction of artifact error induced by soft-tissue movement relative to the underlying bone.

In a static reference position, the locations of markers in a cluster are determined in the global coordinate system, and these are then related to the position of the underlying bone near bony landmarks. Relating the coordinate systems of both the bone and cluster, transformations between the two can be determined. Once the rotations and translations are found, the origin and orientation of the bone-embedded coordinate system are calculated. The next step is to determine the motion the markers undergo relative to the bone over time [Alexander and Andriacchi, 2000].

Without knowledge of bone location over the course of an activity, it is impossible to determine the desired motion. This problem was reduced to an estimate that imposed a functional form of the marker trajectories relative to the bone. This can be formed from the knowledge of the motion of the planned activity. For example, periodic activities can model marker motion relative to the bone as a sinusoidal activity. However, this only represents general characteristics of anticipated deformation [Alexander and Andriacchi, 2000] and, as it turns out, a successful application of this method is highly dependent on the assumed modeling form [Alexander and Andriacchi, 2000; Stagni, et al., 2003].

The data presented from an in vivo study using an Ilizarov device shows significant reduction of error resulting from this method over one using the data of the study as a rigid body model. Represented numerically, the average location error was reduced from $0.25 \mathrm{~mm}$ to 0.08 
$\mathrm{mm}$. However, such small errors can be attributed to the nature of the Ilizarov device [Alexander and Andriacchi, 2000], or pin-mounted devices in general, which may limit the skin motion near the insertion point of the pin. When using the same device in the OCST validation study, Taylor, et al., were careful to allow soft-tissue motion by introducing small incisions around the screws to free any anchoring of the tissue [Taylor, et al., 2005].

A follow up study was performed to validate this technique and its PCT predecessor using multiple subjects and videofluoroscopy instead of using an external fixation device. The anatomical reference frame was determined by the CAST method, as described earlier. The results showed how comparable the two methods were in terms of mean errors in three directions, on the order of $1 \mathrm{~mm}$, but modeled as a cubic function, the IDT produced much larger standard deviations than the PCT. A Gaussian model of the IDT was used as well, but the results were not plotted due to the large degree of error still present after analysis.

Similar in conclusion to other available methods, this technique did not provide any substantial improvement of artifact reduction [Stagni, et al., 2003]. A benefit inherit in the IDT method is the ability to adapt to varying activities and subjects through model estimation [Alexander and Andriacchi, 2000], but unfortunately these functions are not readily known for routine motion analysis for different subjects [Stagni, et al., 2003].

\subsection{Conclusion}

Though many methods have been developed in efforts to reduce, if not eliminate, the soft tissue artifact errors in motion studies, arguably none have been successful in substantially improving upon other available techniques to accurately predict skeletal motion. With this error still a viable influence on the analysis of these studies, it will always be difficult to describe completely the motion of the underlying bone given any activity. 


\section{Chapter 3. Modeling the Dynamic Motion of Soft Tissue}

\subsection{Introduction}

The Kalman filter and sprung mass equations are introduced to approach the modeling of the motion of bone and soft tissue. The Kalman filter is a signal-processing algorithm applied to estimating bone position and orientation data from multiple sources mounted on sprung masses. The sprung mass equations determine the distance separating the tissue surface and the underlying bone. Basic terms are described in the sections pertaining to each of these methods.

\subsection{Kalman Filter}

\subsubsection{Definition}

The Kalman filter is a method that optimally processes recursive data [Maybeck, 1979] and conditions the current estimate using all previous measurements [Welch and Bishop, 2001]. In simpler terms, this technique estimates the current value of a variable making use of all input measurements from various sources. The information considered also includes device dynamics, noises, errors, uncertainty in models, and initial conditions [Maybeck, 1979].

\subsubsection{Process}

The unique ability of this technique is the handling of error and noise within the processes and measurements. This random noise is statistical and should be handled with stochastic methods [Welch and Bishop, 2001] because 1) mathematical models are merely approximated and have varied uncertainties, 2) dynamic systems are affected by uncontrollable disturbances that cannot to be modeled deterministically, and 3) sensors do not produce perfect data and are typically corrupted with noise [Maybeck, 1979]. Though these noises seem, at first, to be unrestricted through their existence in the data, the Kalman filter may be applied only if the noise meets certain restrictions. 
The noises considered throughout this process must be white and Gaussian. Whiteness means that the noise is independent over time and that its value at one instant has no influence on that from another instant [Welch and Bishop, 2001; Maybeck, 1979]. Gaussian distribution of the noise amplitude for its probability density assumes that the collection of noise from multiple sources results in a bell-shaped curve [Maybeck, 1979]. It can be shown that the sum of a given number of random variables under any distribution will result in a Gaussian distribution about the mean value [Welch and Bishop, 2001].

From random variable measurements, the first and second statistical moments, mean and variance, respectively, are typically known and completely define a Gaussian density [Welch and Bishop, 2001; Maybeck, 1979]. The mean is the expected value of a random variable. The magnitude of the variance hints at how much noise is in a signal, but does not show the rate at which this noise occurs [Welch and Bishop, 2001]. A more attractive representation of density distribution is the standard deviation, which directly corresponds to the uncertainty of the measurement and the broadness of its peak [Maybeck, 1979] (see Figure 3.1). This broadness shows how great the chances are that the measurement has a particular value.

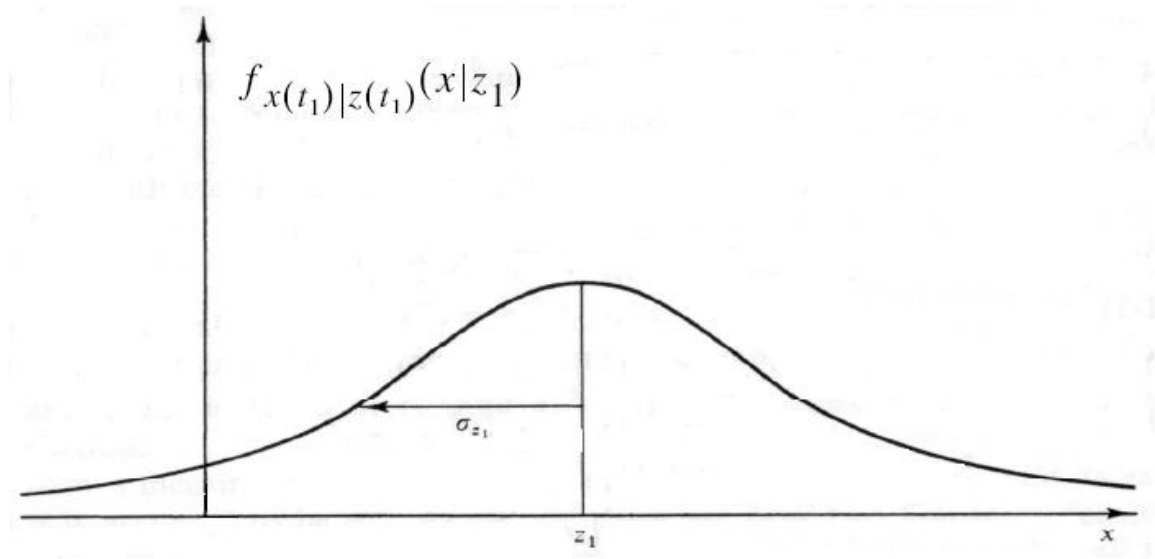

Figure 3.1. Conditional density of a sample measurement [Maybeck, 1979]. 
Using the data obtained from one source will yield results with an accuracy and variance limited to those of the data source. However, considering measurements of a variable from multiple data sources has the ability to increase the accuracy of results and reduce the variance of the calculated mean. Maybeck [1979] demonstrated this in an example involving position measurements from two sources at one time.

For each instant in time, having knowledge of data from two sensors gives a sense of the range of possible values a measured variable may have. Being able to determine which of the two measurements is more accurate and weighing the two appropriately so that a new, calculated value with lower variance is determined is the purpose of the Kalman filter (see Figure 3.2). The variance, or the square of the standard deviation, of each sensor along with the measurement values determine the optimal calculated variable estimate for that instant in an equation resembling the Lever rule:

$$
\mu=\left[\frac{\sigma_{z_{2}}^{2}}{\sigma_{z_{1}}^{2}+\sigma_{z_{2}}^{2}}\right] z_{1}+\left[\frac{\sigma_{z_{1}}^{2}}{\sigma_{z_{1}}^{2}+\sigma_{z_{2}}^{2}}\right] z_{2}
$$

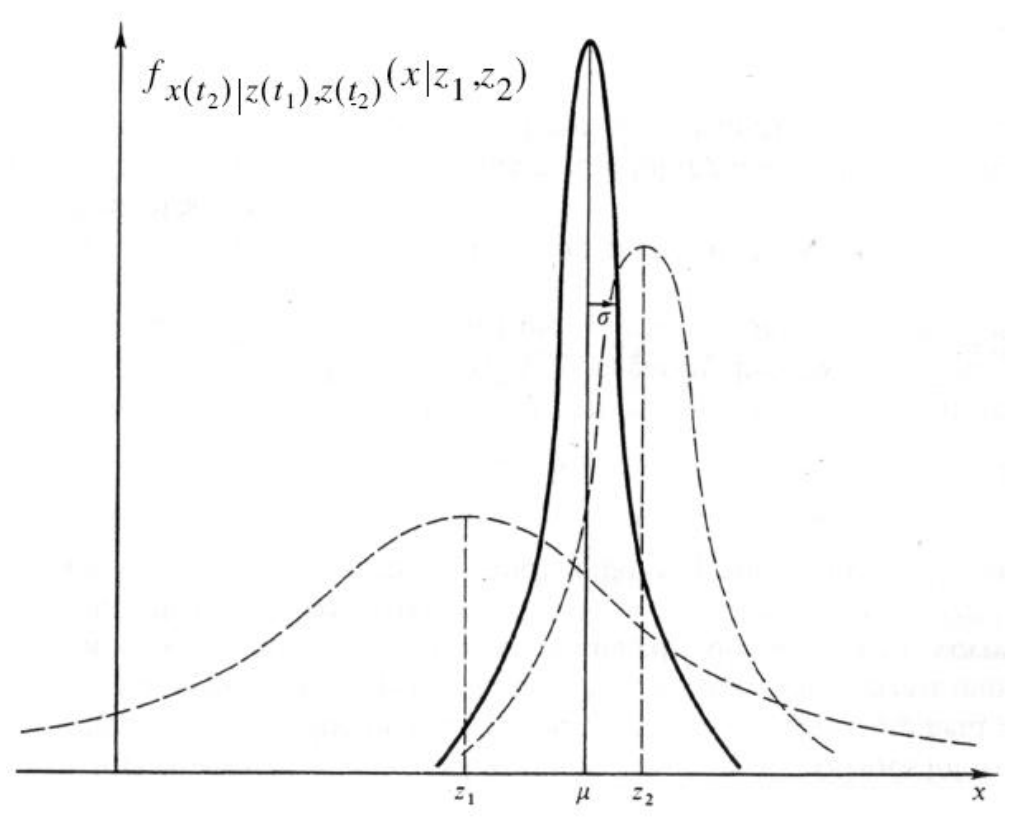

Figure 3.2. Calculated mean from two data sets using the Kalman filter [Maybeck, 1979]. 
where $z$ and $\sigma_{z}$ are source measurements and variances, respectively, for the two sources and $\mu$ is the new calculated variable estimate. The new variance resulting from the calculated mean is as follows:

$$
\frac{1}{\sigma^{2}}=\frac{1}{\sigma_{z_{1}}^{2}}+\frac{1}{\sigma_{z_{2}}^{2}}
$$

Rewriting the mean, $\mu$, in Equation 3.1 so that it reflects the estimation variable commonly used in Kalman filters, $\hat{x}$ :

$$
\hat{x}\left(t_{2}\right)=\hat{x}\left(t_{1}\right)+K\left(t_{2}\right)\left[z_{2}-\hat{x}\left(t_{1}\right)\right]
$$

where

$$
\begin{gathered}
K\left(t_{2}\right)=\frac{\sigma_{z_{1}}^{2}}{\sigma_{z_{1}}^{2}+\sigma_{z_{2}}^{2}} \\
\hat{x}\left(t_{1}\right)=z_{1}
\end{gathered}
$$

This weight, $K$, known as Kalman gain, is incorporated in the calculation to act as a correcting term following the measurement from the first source. This approach leads to this filter having a predictor-corrector operation [Welch and Bishop, 2001; Maybeck, 1979]. Following Equations 3.3 and 3.4, the greater the variance of the first source becomes, the more heavily the second source measurement source is weighed. The opposite happens as the variance of the first source decreases to a value below that of the second.

Processing the signal at each instant from both sources using this filter will increase the accuracy and confidence of the results as compared to the use of only a single source. This results from decreased variance in the estimated value and proper weightings based on individual sensor properties. 


\subsection{Artifact Calculation}

\subsubsection{Modeling the Body}

The rigid skeletal body is a complex system to analyze while in motion. Attached to it are varying amounts of soft tissue moving in different directions and at different rates. The movement of these soft tissues, comprised of tendons, ligaments, muscles, fat, and skin does not accurately mimic that of the skeletal body and therefore cannot accurately portray how the body moves.

The motion of the tissue does not have unlimited range. Its restriction comes from its elasticity and stretched length over the bone. The soft nature of the tissue allows it to spring back to an equilibrium position when a disturbing force is applied and later released. Therefore, the vibrating mass of tissue over a rigid body of bone is modeled as a visco-elastic system undergoing motion relative to a rigid base.

\subsubsection{Artifact Reduction}

Modeling soft tissue as a sprung mass system over the bone allows for simple dynamic modeling for the calculation of bone position and its motion over time. Newton's second law of motion governs the basic system, shown in Figure 3.3:

$$
\sum F=m \ddot{x}
$$

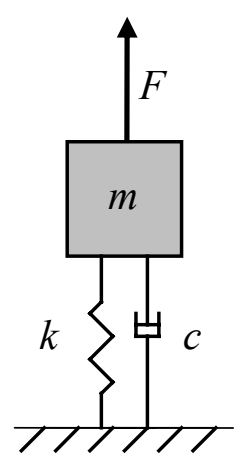

Figure 3.3. Visco-elastic model of soft tissue and a vibrating mass. 
where $m$ is the sprung mass, $F$ is the sum of forces acting upon the mass, and $\ddot{x}$ is the acceleration of the mass. This system has only a single degree of freedom because of its restriction of motion only along the vertical direction. Breaking down Equation 3.6 further to account for the spring and damper components (Figure 3.4):

$$
F=m \ddot{x}+c \dot{x}+k x
$$

where $c$ is the damping coefficient, $\dot{x}$ is the mass velocity, $k$ is the spring constant and $x$ is the mass displacement along the axis of the spring and damper.

Equations 3.6 and 3.7 work well when the mass is moving relative to a stationary base. However, the bone, modeled as the base, also undergoes motion. To account for this extra motion, the equations are altered.

The spring and damper components only contribute a force to the system when the distance between the mass and base changes from their free length, $\rho$. Defining base position as $z$ and mass position as $x$ (see Figure 3.5):

$$
y=x-z-\rho
$$

and Equation 3.7 becomes:

$$
F=m(\ddot{y}+\ddot{z})+c(\dot{y}+\dot{z})+k(y+z+\rho)
$$

For both the damper and spring, the $z$ component is removed along with $\rho$ because $y$ is the only factor pertaining to the forces being exerted as already explained. Assuming no external forces

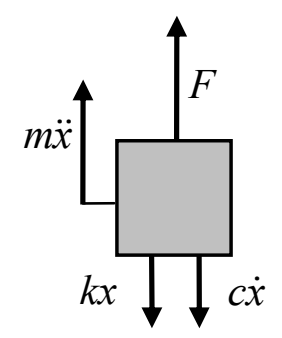

Figure 3.4. Forces present in visco-elastic model. 


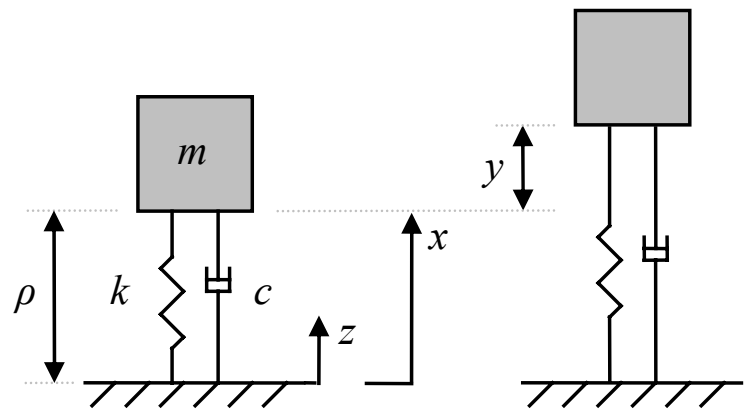

Figure 3.5. Base and surface motion with respective displacement origin.

are present, $F$ is removed as well. Thus, the differential equation governing the system of soft tissue in motion is left:

$$
m \ddot{y}+c \dot{y}+k y=-m \ddot{z}
$$

This is the equation presented by Trujillo and Busby [1990], though it does not solve for the displacement of the surface of the soft tissue, $y$, in this form. A simplification in the current work is the absence of a damping element. Because of this, its term is removed and the equation is simplified to the following:

$$
y=\frac{\ddot{x}}{-\omega^{2}}
$$

where

$$
\omega^{2}=\frac{k}{m}
$$

Finally, using the position of the mass, $x$, defined in Equation 3.3, the known spring free length, $\rho$, and the artifact, $y$, defined in Equation 3.11, the position of the base, $z$, over time is found using Equation 3.8.

These equations state that the artifact of motion is found using only the recorded axial acceleration and with knowledge of the system spring constant and mass. The most complicated of the variables to determine is the tissue spring constant. This value will differ within a 
population and within each subject over a motion and therefore in situ measurements would need to be taken to determine instantaneous spring constant values [Trujillo and Busby, 1990].

In a controlled experiment with a constant spring rate known, further described in Chapter 4, the approach of determining bone position and motion from accelerations recorded on the tissue surface is validated. 


\section{Chapter 4. Design of the Test Station}

\subsection{Introduction}

The design of an experimental station to represent motion of a tissue-covered body was necessary to further understand fundamental skeletal movement. This station was fabricated so that cyclical motion could be produced over time similar to that of a typical animal's limb, however, only in the sagittal plane. Attached to the limbs were sprung masses representing soft tissue, which had two sensors independently measuring the acceleration and position of the masses at the end of the springs.

\subsection{Anatomical Simulation with Cyclical, Repetitive Motion in Two Dimensions}

Two key goals were fulfilled in the design of the experimental test station. The first was to produce a motion representative of an animal's tissue-covered limb, which mainly occurs in a plane. The second was the need to reproduce the motion over time through numerous cycles.

The literature simplifies the analyses and generalizes the anatomy of the bones and soft tissue as well as the motion of the joints. It is believed that, like swinging limbs in many other organisms, those of a horse have evolved to move primarily in the sagittal plane [Back, et al., 2001] and therefore move like a pendulum about its proximal joints [Back, et al., 1995(1); 1995(2)]. From individual inspection, a similar conclusion of planar motion can be assumed for a variety of animals such as humans and canines during a walk. For these cases, even though bone motion occurs in all three dimensions, it is evident that the majority of the motions are planar and the rotations of the joints are primarily about a single axis.

Although the limbs in all of the aforementioned animals demonstrate their primary motion to be in the sagittal plane, there remains a lesser amount of out of plane rotation. Figures 4.1 and 4.2 demonstrate the relative amounts of limb travel in and out of the sagittal plane. 


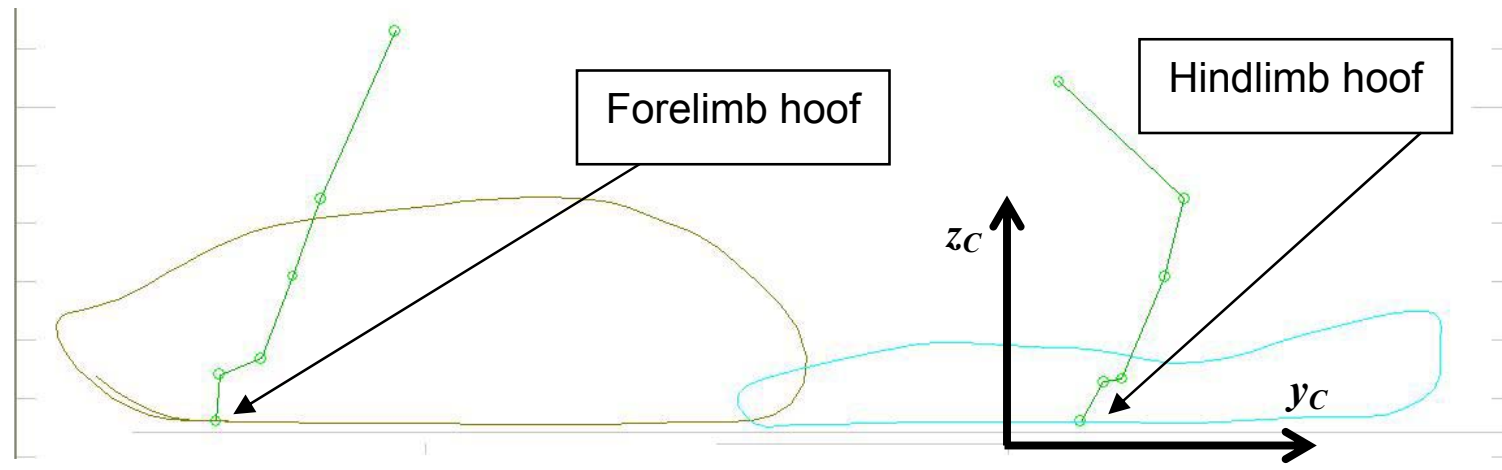

Figure 4.1. Path of hind- and forelimb equine hooves in the sagittal plane during one cycle.

Specifically, these figures show the travel of two hooves over one complete canter cycle of a Paso Fino horse, named Roy, on an equine treadmill at the LSU School of Veterinary Medicine during initial experiments with the Codamotion ${ }^{\mathrm{TM}}$ system and software. Active markers using LED's were located at strategic locations along the lateral side of both the left hind- and forelimbs. The significant differences in range of motion of both sets of limbs between the two viewing planes show how modeling limb rotation about a single axis can be justified. However, in many animals such as humans, it should be noted that even though the gait cycle may be constrained mainly to two-dimensional motion, the joints are capable of more degrees of freedom given different tasks.

As animals undergo motion, a combination of motions from multiple body segments is repeated continuously. A representative path of one point is shown in Figures 4.1 and 4.2. This

Figure 4.2. Path of hind- and forelimb equine hooves in the frontal plane. 
fundamental motion characteristic remains typical over a variety of speeds ranging from a slow walk to a fast gallop though the stride length or cycle frequency may change. Even the course of limb travel is subject to change to improve the efficiency of motion.

The requirement of cyclical, repetitive motion in two dimensions was used as a fundamental basis for the design of the test station. This offers basic characteristics common among animals so that this experiment can provide general expectations of future studies using three-dimensional motion or those performed in vivo.

\subsection{Test Station Construction}

The test station was designed and constructed using the previously described requirement along with the representative components of the fundamental tissue-covered animal body. The components include a limb, comprised of the skeletal structure and soft tissues, as well as the connecting joints (see Figure 4.3).
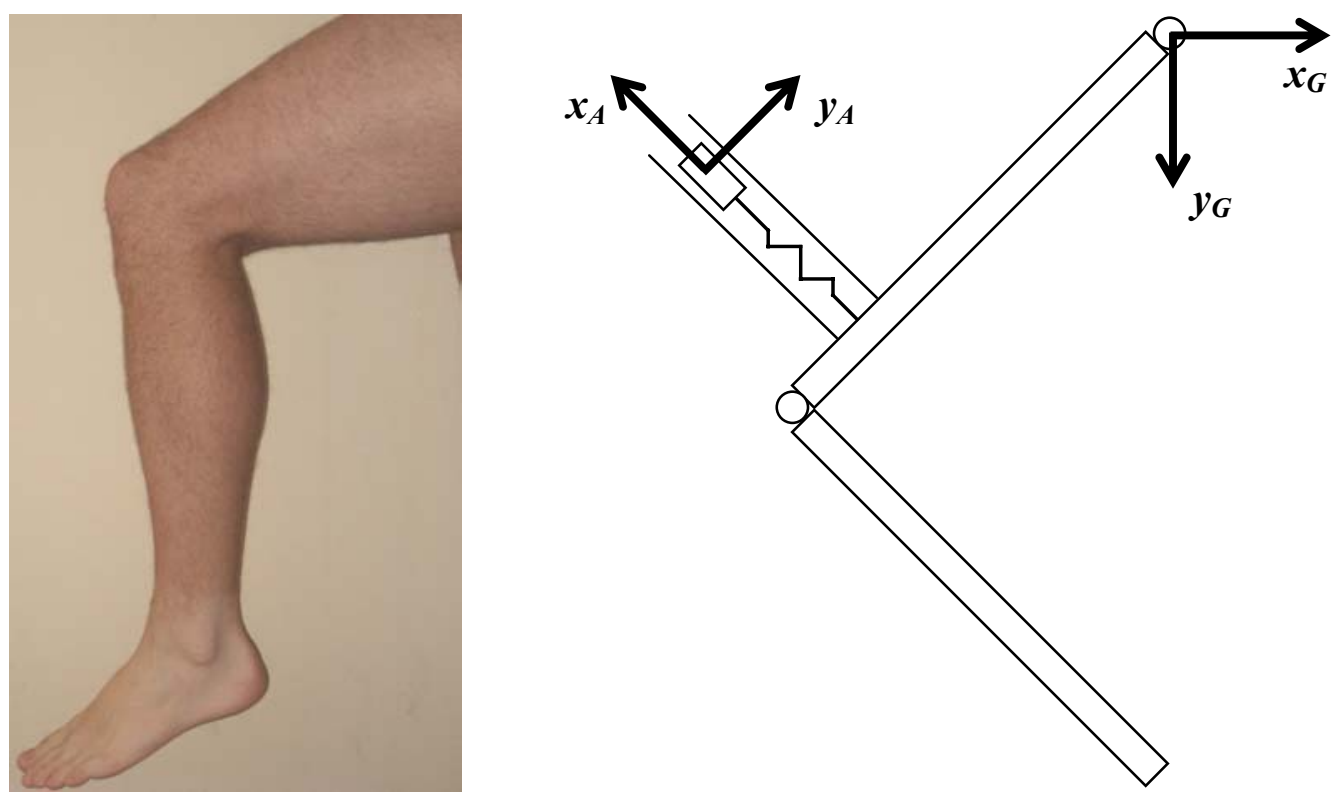

Figure 4.3. Representation of animal body in the test apparatus. 


\subsubsection{Experimental Configuration of Anatomical Representations}

The limbs were modeled as rigid bodies, both lightweight and rigid. In a simple representation of a walking limb in an animal, only two links arranged as a double pendulum were used. These links model the femur and shank, the two longest and largest members of the lower body in humans (see Figure 4.4). Though only two links were used, this design is not limited to only these two members, as additional units may be added to represent other components.

The soft tissue is represented using two connected, yet separable, components, a spring and a mass. The spring represents the elastic properties of the tissue, both extensive and compressive, constrained to the axis perpendicular to the bone using a Teflon ${ }^{\circledR}$ tube. The mass, a Teflon $^{\circledR}$ cylinder, represents the mass of the tissues along the bone in a concentrated element. The spring constant was fixed in this experiment, unlike the varying rates in animal bodies due to varying amounts of different tissues at different locations of the body as well as variation in

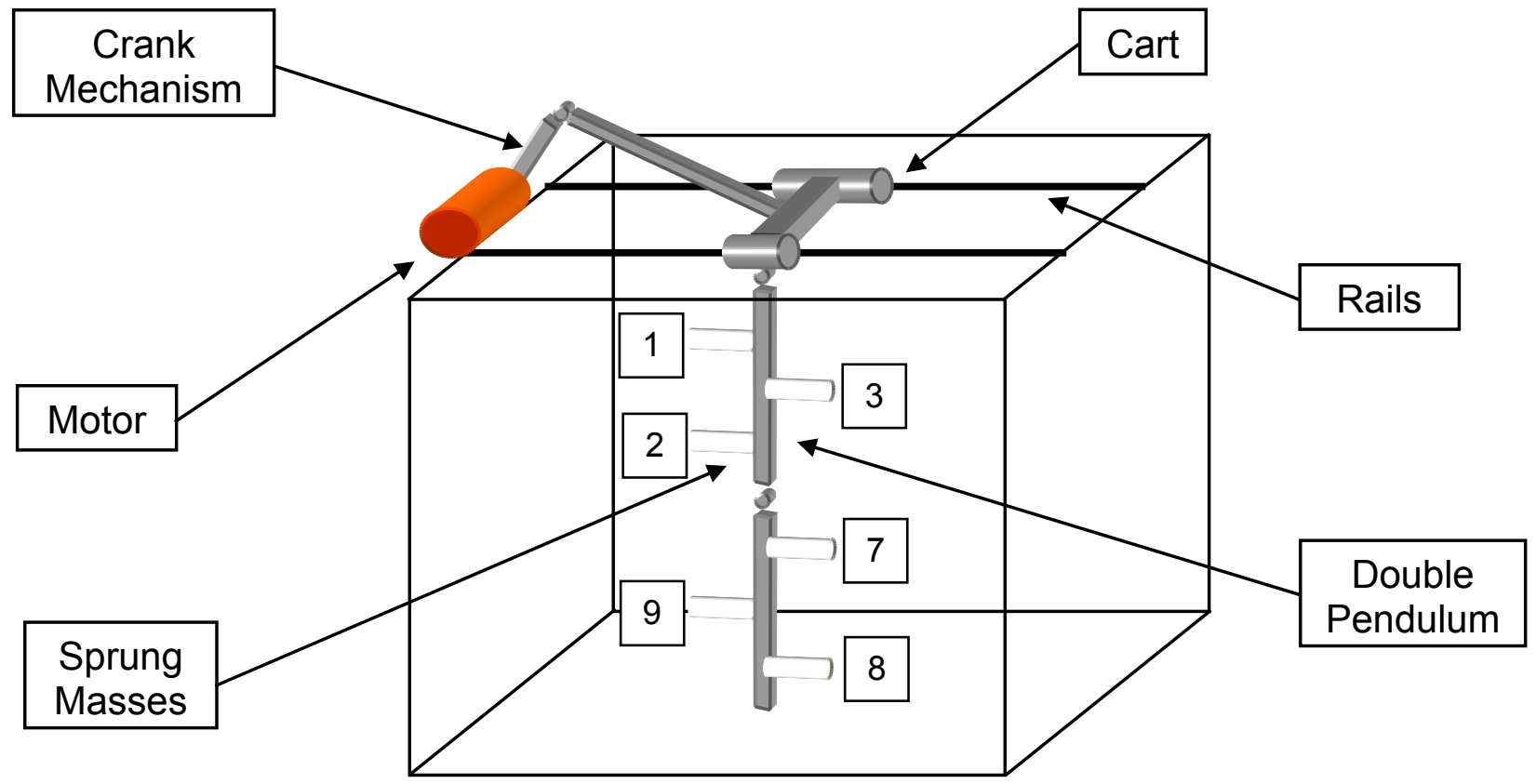

Figure 4.4. Schematic of test station. 
elastic properties due to the firings of muscles. The spring rate was chosen using a kinematic analysis of a slider-crank mechanism such that the mass would experience a range of travel similar to those presented in previous studies under accelerations several times that of gravity.

The joints are represented at the interface of the two rigid bodies by a shaft acting as the axis of rotation. This shaft passes through the top portion of a shoulder joint firmly connected to the upper link. The shoulder surrounds the lower portion and is firmly connected to the lower link. As the shaft passes through the joint members it is tightly held to the lower portion with a set screw and freely rotates using needle roller bearings. The low resistance mimics the natural system in synovial joints.

The double pendulum was put into motion using a simple crank mechanism. Holding the top joint of the system, similar to the hip, is a cart that slides horizontally along a rail assembly using linear ball bearings (see Figure 4.5). The cart is attached to the crank mechanism and driven by a $120 \mathrm{VAC}$ drill motor. The motion induced by this mechanism produces movement

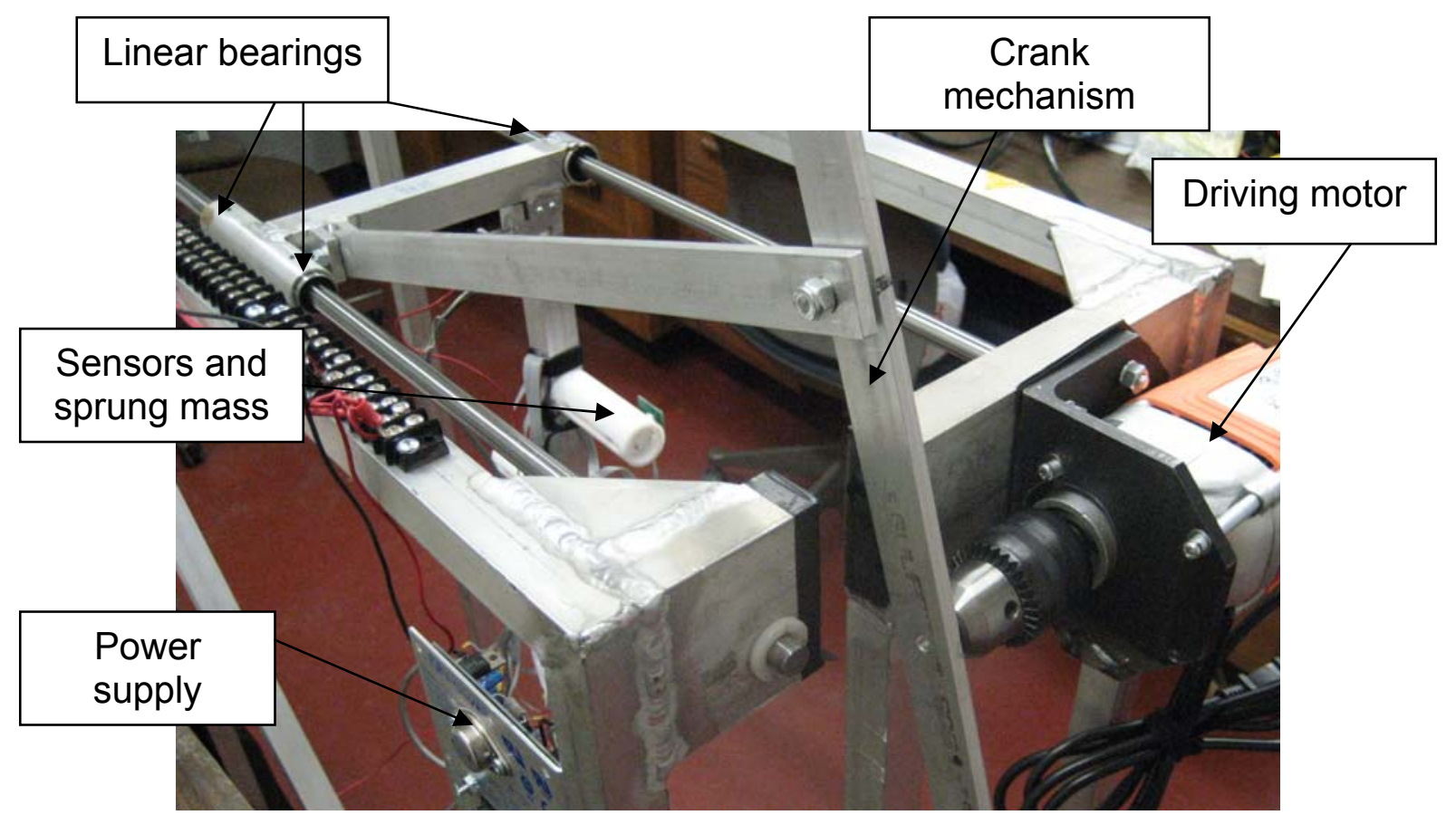

Figure 4.5. Experimental apparatus with simple crank mechanism. 
of the double pendulum and displacement of the masses. The collective motion of this experimental station simulates typical animal motions in common activities.

\subsubsection{Sensor System}

Attached to each mass were two types of sensors to track the location over time (see Figure 4.6). The first sensor attached to the mass was a two-axis ADXL210 accelerometer (Analog Devices, Norwood, MA) powered by a 5 VDC, linear power supply (International Power, Oxnard, CA). This accelerometer was aligned on the mass such that one of its axes was parallel to the spring axis and the other was parallel to the link. Transforming the accelerations collected in the local coordinate system by LabVIEW 7.1 (National Instruments, Austin, TX.) to global coordinates and integrating allowed for determination of the position of each mass relative to the global coordinate system origin. The second sensor was an active LED marker that was tracked using the Codamotion ${ }^{\mathrm{TM}}$ system. These sensors were attached to the sprung mass with the accelerometer and to the pendulum link at the base of each mass. This system calculated the real-time three-dimensional position of each marker over time using a CODA sensor unit with three detecting elements, two for horizontal positioning and one for vertical. As previously described, these two data streams were processed using the Kalman filter to more accurately estimate the location of each mass over the course of an experiment.

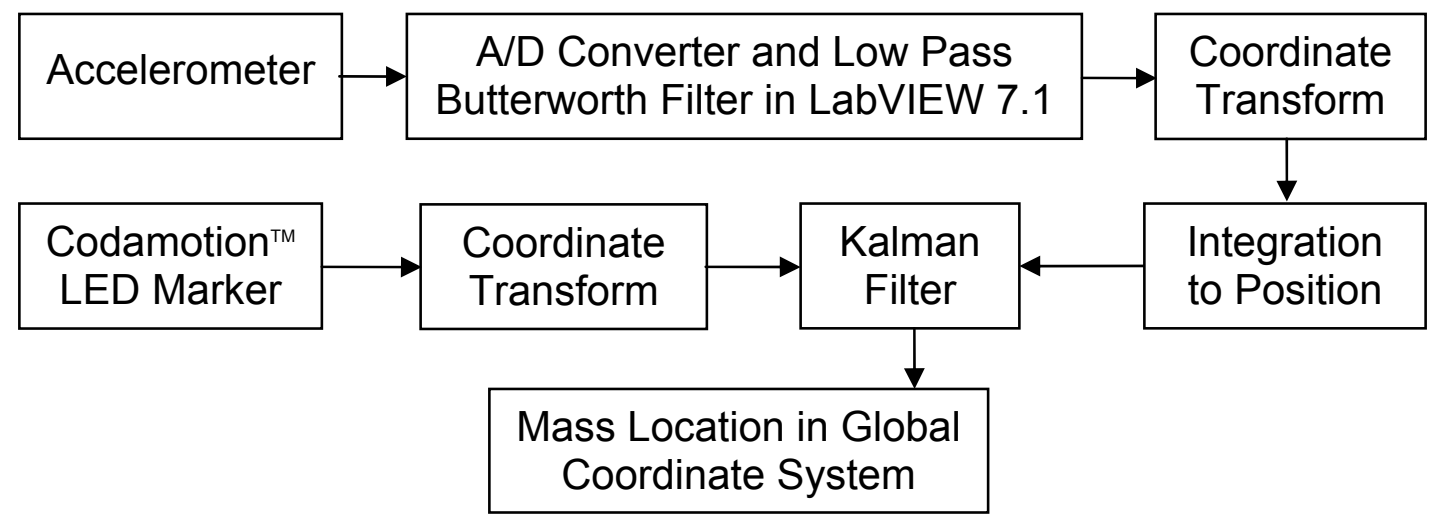

Figure 4.6. Steps for the collection of position data. 
To transform from the local coordinate system to the global, the joint angle must be determined. The angular measurements were determined using the shaft of the joint, which is part of a Honeywell 43C120 potentiometer (Honeywell Inc., Freeport, IL). With a 5 VDC reference voltage providing an input, the rotation of the shaft due to relative motion of the lower link (see Figure 4.7) generates a voltage that is linearly proportional to the shaft angle as shown in Equation 4.1:

$$
\theta_{J o \text { int }}=R_{P o t .} \times \frac{V_{D A Q}-\left(V_{o}-V_{\operatorname{Re} f \cdot / 2)}\right.}{V_{\operatorname{Re} f .}}-\frac{R_{P o t .}}{2}
$$

where $R_{P O t}$ is the total angular range of the potentiometer, $V_{D A Q}$ is the output voltage acquired by the data acquisition card, $V_{o}$ is the initial voltage of the potentiometer output to set the motionless angle of zero, and $V_{\text {Ref. }}$ is the reference voltage. Ideally, the value for $V_{o}$ was $2.5 \mathrm{~V}$ corresponding to the shaft being at the midpoint of rotational range, but during assembly the shaft was rotated, changing this value and needing compensation to guarantee proper angle measurements.

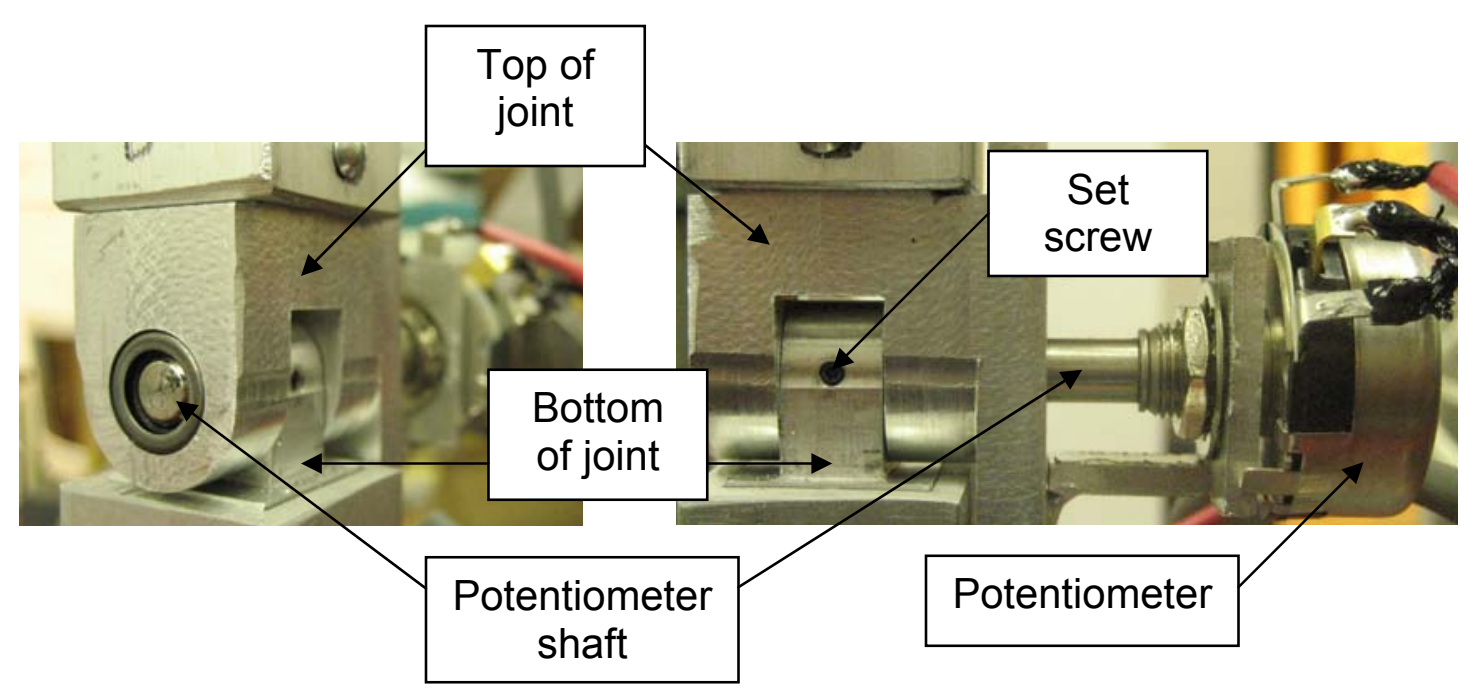

Figure 4.7. Mechanical joint and potentiometer. 


\subsection{Conclusions}

Using the experimental station described in this chapter, cyclical motions representative of a tissue-covered body were modeled in two dimensions and were performed. This was done using a simple crank mechanism and a double pendulum with attached sprung masses restricted to motion perpendicular to each pendulum. The sensors attached to the sprung masses collected two-dimensional acceleration and position data during these motions. The position data for the link at the base of each sprung mass were collected as well. The recorded data were analyzed for completeness and used for validation of this process. 


\section{Chapter 5. Experimental Results and Analysis}

\subsection{Introduction}

A test stand was designed and fabricated to collect measurements of representative tissue motion to be analyzed for determination and computational validity of this approach in resolving bone position dynamically over time. Multiple coordinate systems, sensor and global, were used in data measurement and calculation of both the soft tissue and base motions. The results of this research are presented in the following sections with implications for its usefulness in future studies.

\subsection{Coordinate Systems Used in Experimental Analysis}

\subsubsection{Sensor-Based Coordinate System}

The accelerometer-based coordinate systems originated on each of the accelerometers, which were positioned on the sprung masses recording accelerations of the simulated soft tissues (see Figure 5.1). The accelerometers were positioned such that the $\mathrm{x}$-axis was in-line with the spring axis and the y-axis was parallel to the pendulum. The positive direction for each during acquisition depended on which side of the pendulum the sensor was located and is portrayed in Figure 5.1. For analysis, the sign conventions for the data were changed so that positive

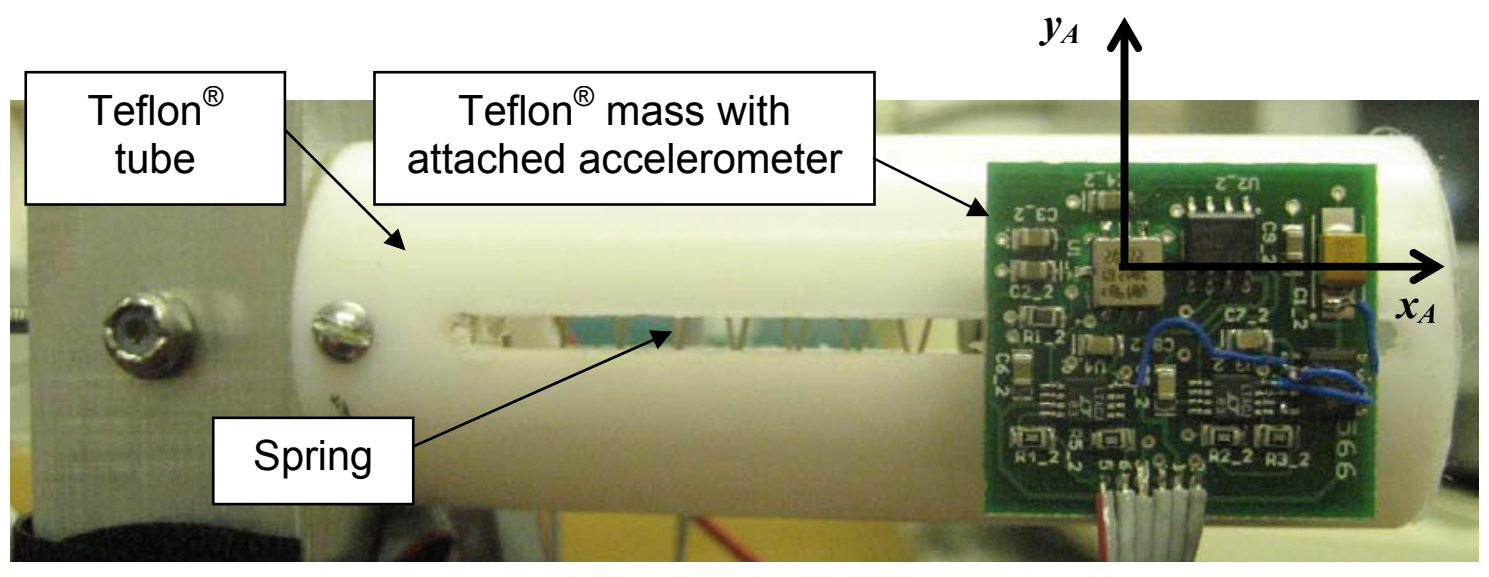

Figure 5.1. Accelerometer-defined coordinate system. 
orientation for the $\mathrm{x}$-axis was in the spring extension direction and the $\mathrm{y}$-axis projected toward the top joint in the mechanical system.

Unlike the accelerometers, which each defined an individual coordinate system, the CODA system provides the three-dimensional coordinates of each active marker in the field of view of its camera-like sensors in a comprehensive, system-defined coordinate system (see Figure 5.2). The origin was located by the marker at the location of the application of the driving force, the top joint, simulating the hip and its motion.

\subsubsection{Global Coordinate System}

The global coordinate system provided the basis for which motion data from each sensor type was related. The origin of this system was located by the marker at the CODA system origin shown in Figure 5.2 as the top joint. However, the positive directions of the global axes

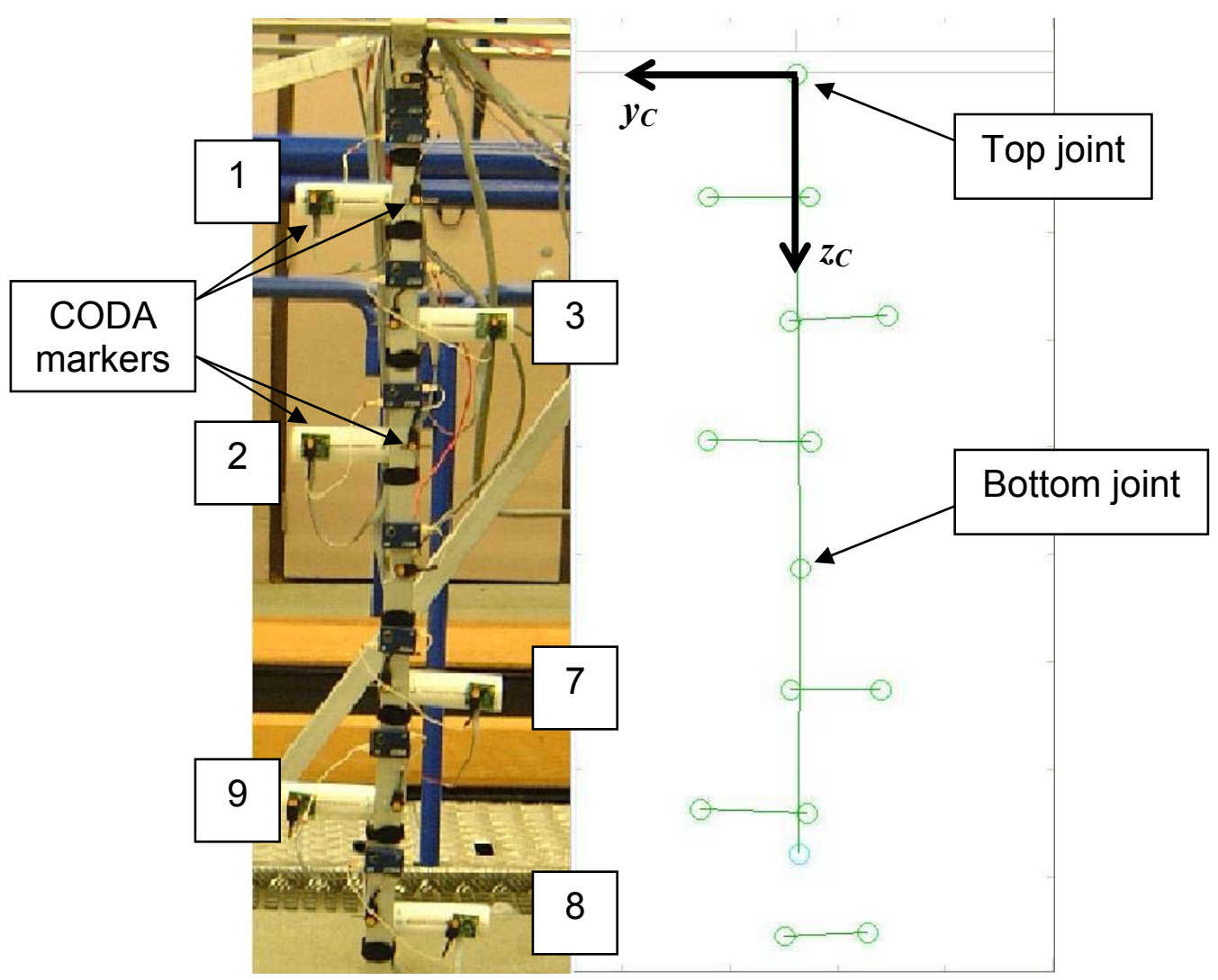

Figure 5.2. CODA-defined coordinate system. 
do not reflect those from the CODA. The global directions defined the positive $\mathrm{x}$-axis to be the customary left-to-right direction and the positive y-axis to be in the direction of pendulum extension, or vertically down. This origin provided a constant datum for the travel of its $\mathrm{x}$-axis as its vertical travel at this location was unchanged throughout motion.

Coordinate transformation of both data sets were necessary to properly combine the data from the two sensor types and provide the finalized position coordinates in a single coordinate system. Figure 5.3 demonstrates the transformation of the accelerometer-based coordinate system and is mathematically represented in Equation set 5.1. Figure 5.4 and Equation set 5.2 demonstrate the transformation for the CODA.

$$
\begin{gathered}
{\left[\begin{array}{c}
\ddot{x}_{G} \\
\ddot{y}_{G}
\end{array}\right]=\left[\begin{array}{cc}
\cos \theta & -\sin \theta \\
-\sin \theta & -\cos \theta
\end{array}\right]\left[\begin{array}{c}
\ddot{x}_{A} \\
\ddot{y}_{A}
\end{array}\right]} \\
{\left[\begin{array}{c}
x_{G} \\
y_{G}
\end{array}\right]=\left[\begin{array}{c}
-y_{C} \\
z_{C}
\end{array}\right]}
\end{gathered}
$$

\subsection{Soft Tissue Motion}

\subsubsection{Collected Position Data}

The data from the CODA active marker system provided a direct measurement of the sprung marker position over time in the global coordinate system using the predefined origin at

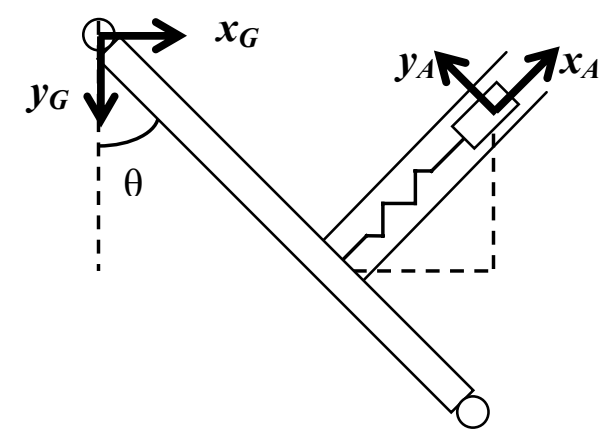

Figure 5.3. Accelerometer coordinate system transformation. 


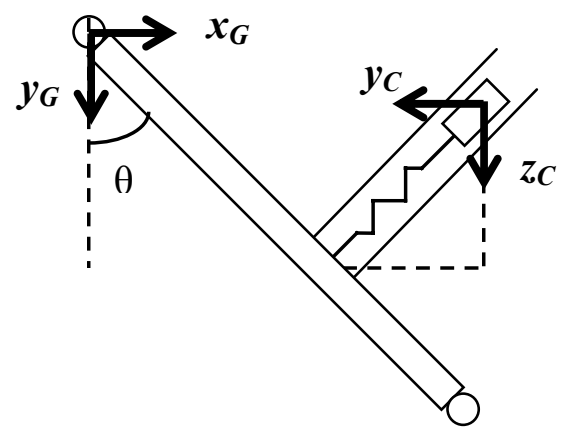

Figure 5.4. CODA coordinate system transformation.

the top joint. For each marker, two data streams were collected over the time during which motion occurred, one for each of the global axes, $\mathrm{x}$ and $\mathrm{y}$. This data were input to the Kalman filter.

The collected acceleration data were used to track position of each mass over time, as well. By integrating this data twice with respect to time, position measurements were made. However, data other than acceleration must be known to locate the position of each accelerometer in space. Initial conditions of the system were known, which included velocity and position in two dimensions for each mass and the joint angles. Starting the system from rest allowed the assumption of zero initial velocity and an initial position as defined by the CODA measurements. Hanging freely, the joint angles were also zero. Beginning with this data allowed for progressive calculation for positions of each sensor.

\subsubsection{Filtered Position Data}

As previously mentioned, the position data acquired from the accelerometers were combined with those from the CODA system for each mass to provide for a more accurate measurement of two-dimensional sprung mass position over time. Using the Kalman filter algorithm discussed in Chapter 3, the resulting position data were calculated for both the $\mathrm{x}$ and $\mathrm{y}$ axes. Figure 5.5 shows the filtered data along with each of the two sensor sets independently. 

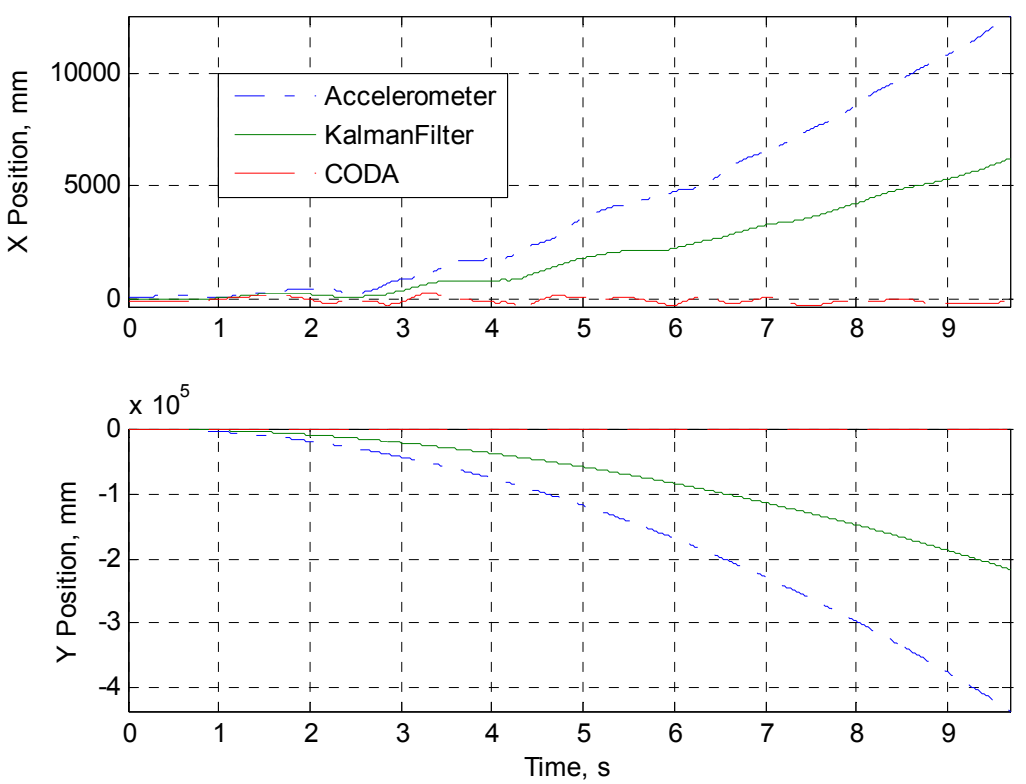

Figure 5.5. Mass position in the $\mathrm{x}$ - and $\mathrm{y}$-directions for position 2 in test 1 .

Under a general assumption that the measurement systems have equal uncertainties, it is seen how the filtered positions are skewed from reasonable values due to the influence of accelerometer drift.

\subsubsection{Drift and Its Effect on Position Measurements}

Drift is a problem associated with many sensors using accelerometers as a source of position measurement. It is an accumulation of errors over time beginning with random bias, or noise, in acceleration data. As this noisy data is integrated, the noise becomes amplified to create an incorrect representation of velocity. As this amplified, noisy signal is integrated one more time, the noise associated with it is amplified as well producing position data with doubleamplified noise signals.

\subsubsection{Reduction of Drift Influence}

As shown in Figure 5.5, as time increases, the reliability of the accelerometer data decreases. One method to reduce the influence of drift is to recalibrate the noisy data using 
external, direct-position measurements [Pang and Liu, 2001; Leach and MacPherson, 1991; Wood, et al., 1997].

The aviation industry relies heavily on the use of accelerometers, independently or with other MEMS sensors, is. These sensing systems help determine aircraft horizontal and vertical velocities and positions during flight. Using filtering techniques, Kalman filter or others, external sources such as a global positioning system (GPS) [Wood, et al., 1997] and Doppler radar [Leach and MacPherson, 1991] have been employed to more accurately measure the velocity and position measurements obtained from accelerometers.

In a more accelerometer-independent study, the drift effect on position data using three distinct acceleration rates was analyzed [Pang and Liu, 2001]. It was concluded that at higher accelerations, a constant bias applied to the accelerometer reduced the integrated position error. However, at lower values, the effect of drift is more influential and a constant bias yielded large discrepancies in position. Therefore, manual tuning of the applied bias in the data was needed over time to provide accurate results. Pang and Liu also report that, for their experiment, a minor drift of $2 \mathrm{mg}$ resulted in a velocity drift of $1.178 \mathrm{~m} / \mathrm{s}$, and a position drift of $35.32 \mathrm{~m}$ over a one minute span [Pang and Liu, 2001] demonstrating how greatly a seemingly insignificant error in acceleration can affect position.

Frequently recalibrating the accelerometers with the CODA sensors, the only measurement system available, would limit the accuracy of the accelerometer data to that of the CODA. The alternative was to disregard the position data obtained from the accelerometers and use only the data from the sprung CODA markers. This data was assumed to be an accurate representation of the position of the sprung mass throughout the experiments. The resulting motion is shown in Figure 5.6. 

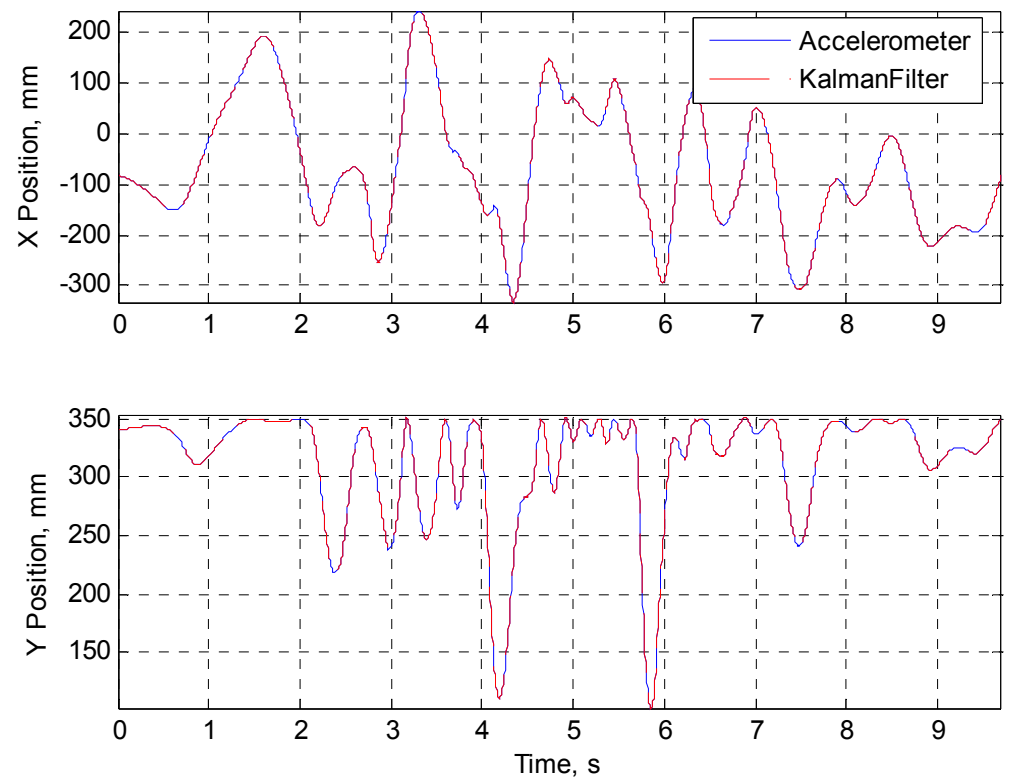

Figure 5.6. Mass x- and y-positions without accelerometer data for position 2 in test 1.

\subsection{Determination of Base Motion}

\subsubsection{Artifact Calculation}

The resolved base motions were calculated with the knowledge of the initial distances separating the base and sprung mass, $\rho_{o}$, the global positions of the masses in two dimensions, and the raw accelerometer data in the sensor coordinate system as explained in Chapter 3.3.2. With $\rho_{o}$ remaining constant for each mass and the positions known with time, the only unknowns were the values of $y$ in Equation 3.11. The $y$ values, in the sensor coordinate systems, were calculated and Figure 5.7 plots these values along with the changes of $\rho$ from the CODA at the same marker location and in the same test presented in Figure 5.5. The results from all six markers during the four conducted tests are located in the Appendix A. It should be noted that the plot of marker number 8 does not resemble those from the CODA readings in any test like the other markers. This is due to the sprung mass and tube making contact and sticking to each other during motion, likely caused by the variation in the clearance between the two from fabrication. 


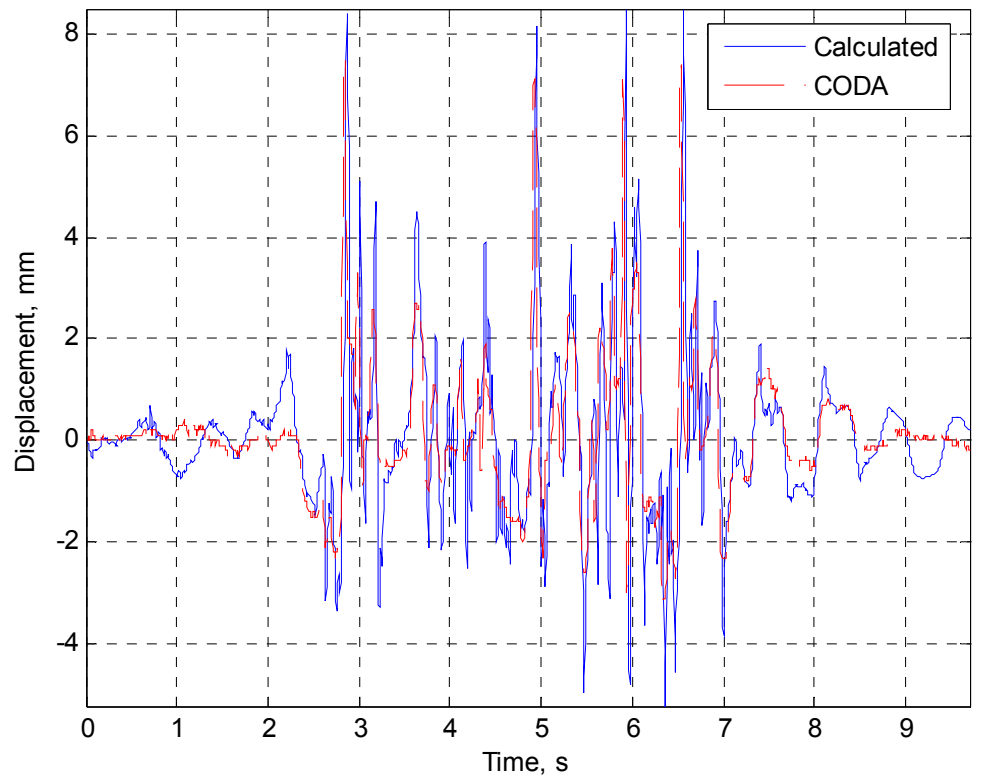

Figure 5.7. Artifact comparison for position 2 in test 1.

The similarity of the two plots demonstrates the accuracy of this approach. The mean differences between the two plots for each of the six marker locations during one test and their standard deviations were calculated (see Figure 5.8). The numeric values for all four of the tests conducted are presented in Table 5.1. Note that over total mass displacements approaching $3 \mathrm{~cm}$, the mean differences between the CODA measurements and the calculated measurements from the accelerometers are typically less than $1 \mathrm{~mm}$, a value less than five percent of the total displacement of the mass. The accuracies of these calculations compared to the artifact values by the CODA markers over the entire travel are in Table 5.2 for all markers in the four tests.

Table 5.1. Mean and standard deviations for artifact differences along spring axis, millimeters.

\begin{tabular}{|c|c|c|c|c|c|c|c|c|}
\cline { 2 - 9 } \multicolumn{1}{c|}{} & \multicolumn{2}{c|}{ Test 1 } & \multicolumn{2}{c|}{ Test 2 } & \multicolumn{2}{c|}{ Test 3 } & \multicolumn{2}{c|}{ Test 4 } \\
\hline Marker & mean & $\sigma$ & mean & $\sigma$ & mean & $\sigma$ & mean & $\sigma$ \\
\hline 1 & -0.19 & 1.40 & 0.09 & 0.89 & 0.56 & 3.11 & 0.19 & 1.68 \\
\hline 2 & -0.03 & 1.28 & -0.02 & 0.81 & 0.43 & 1.58 & 0.02 & 0.70 \\
\hline 3 & 0.18 & 1.29 & -0.16 & 0.56 & -0.18 & 2.88 & -0.12 & 1.36 \\
\hline 7 & 0.11 & 3.41 & -0.20 & 1.26 & -0.14 & 3.59 & -0.19 & 1.66 \\
\hline 8 & -8.05 & 6.86 & 9.72 & 6.00 & -1.74 & 6.13 & 0.13 & 0.50 \\
\hline 9 & 0.11 & 2.41 & -0.03 & 0.83 & 0.66 & 1.33 & 0.01 & 0.54 \\
\hline
\end{tabular}




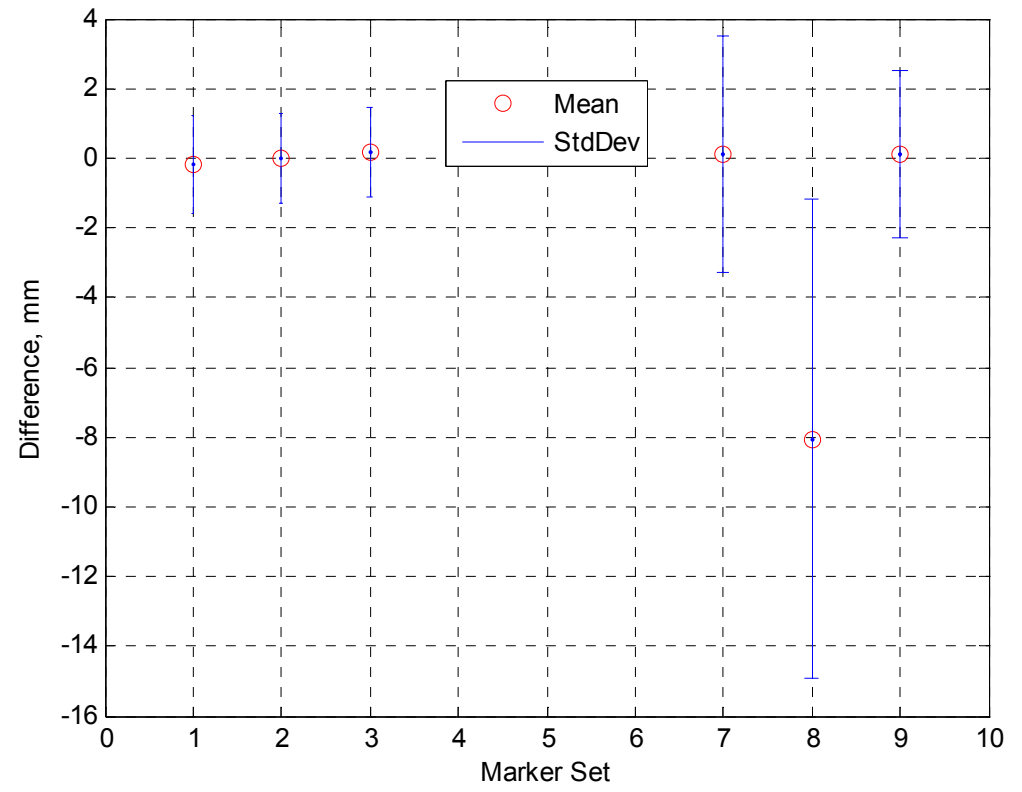

Figure 5.8. Mean and standard deviation in artifact values for position 2 in test 1 , millimeters.

\subsubsection{Base Position Calculation}

The remaining step that was necessary to calculate the position of the base for each time instant involved $\rho_{o}$ and the joint angle with respect to the vertical, $\theta$. Figure 5.9 illustrates the geometry of the system that was used with Equations 5.3 and 5.4 to calculate the desired base positions, $z_{x}$ and $z_{y}$, in the global coordinate system.

$$
\begin{aligned}
& z_{x}=x_{K F}-\left(y+\rho_{o}\right) \cos \theta \\
& z_{y}=y_{K F}-\left(y+\rho_{o}\right) \sin \theta
\end{aligned}
$$

Table 5.2. Artifact range and accuracy of calculated values to CODA measurements.

\begin{tabular}{|c|c|c|c|c|c|c|c|c|}
\cline { 2 - 9 } \multicolumn{1}{c|}{} & \multicolumn{2}{c|}{ Test 1 } & \multicolumn{2}{c|}{ Test 2 } & \multicolumn{2}{c|}{ Test 3 } & \multicolumn{2}{c|}{ Test 4 } \\
\hline Marker & trav., mm & acc., \% & trav., mm & acc., \% & trav., mm & acc., \% & trav., mm & acc., \% \\
\hline 1 & 20.63 & 99.09 & 7.10 & 98.75 & 28.97 & 98.08 & 23.69 & 99.21 \\
\hline 2 & 13.70 & 99.81 & 8.80 & 99.80 & 11.14 & 96.18 & 11.02 & 99.83 \\
\hline 3 & 16.31 & 98.87 & 7.20 & 97.75 & 27.33 & 99.33 & 18.59 & 99.35 \\
\hline 7 & 23.84 & 99.55 & 15.11 & 98.65 & 24.22 & 99.40 & 15.58 & 98.80 \\
\hline 8 & 22.71 & 64.54 & 11.69 & 16.88 & 32.03 & 94.56 & 4.03 & 96.66 \\
\hline 9 & 33.84 & 99.69 & 13.98 & 99.77 & 17.19 & 96.17 & 6.40 & 99.88 \\
\hline
\end{tabular}




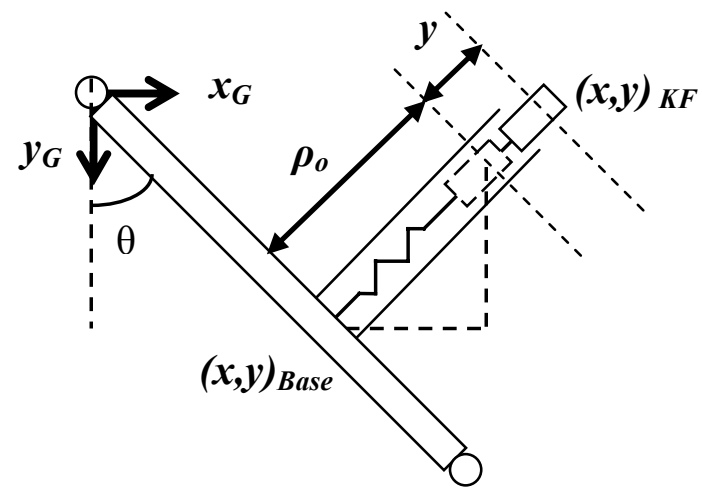

Figure 5.9. Base coordinate calculation.

There were two CODA markers for each of the six accelerometers, one was placed on top of the accelerometer and the other was on the pendulum at the base of the spring. The distances separating the two varied at each location giving a unique $\rho_{o}$ for each. However, since the two were not necessarily the same vertical distance from the origin, an artificial vertical "relocation" of the base marker was necessary to provide accurate measurements and comparisons of $x_{G}$ and $y_{G}$ base positions. This relocation modified the $y_{G}$ data of the base markers so that they appeared to be aligned in the vertical axis at a joint angle of zero. The geometry is shown in Figures 5.10 and 5.11 for markers on both sides of the pendulum and the equations for relocation are shown in Equations 5.7 and 5.8.

$$
\begin{gathered}
\Delta y_{G, o}=y_{G, o \text { Base }}-y_{G, o \text { Sprung }} \\
y_{G, \text { Base }}=y_{G, o \text { Sprung }}+\Delta y_{G, o} \cos \theta
\end{gathered}
$$

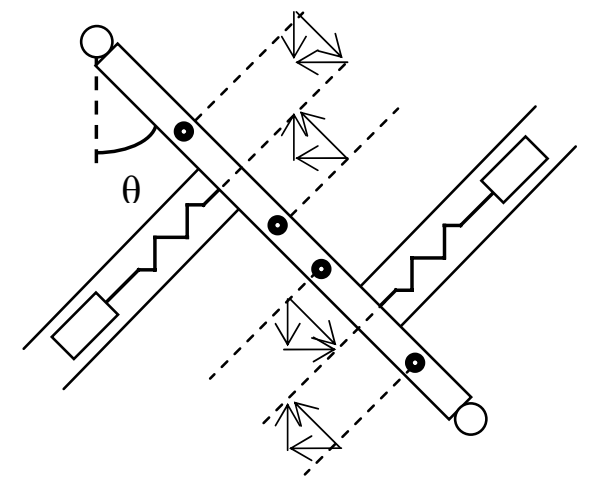

Figure 5.10. Artificial relocation of base markers with vertical offsets. 


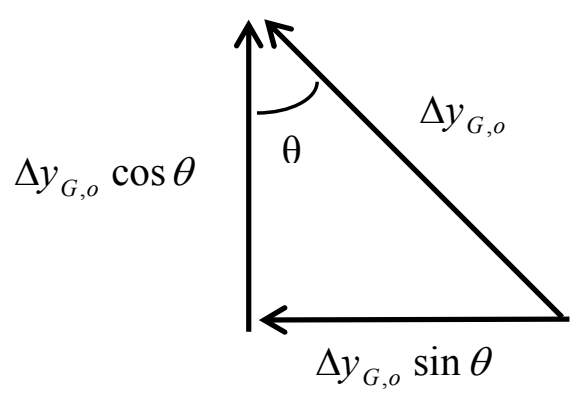

Figure 5.11. Relocation of base marker vertically below a sprung marker.

In plotting the motion of the base at all six marker locations, the similarities between the position of each CODA marker at the base and the calculated value from the mass are seen again. This is because both mass sensors begin at a distance of $\rho_{o}$ from the base marker and the two artifact data sets are nearly identical. The base for one of the markers during one test is shown in Figure 5.12 and all four of the tests conducted are presented numerically in Tables 5.3 and 5.4. The plots from all six markers during the four conducted tests are in Appendix B. For the instance shown in Figure 5.12, the mean differences between the base positions determined by the accelerometer data and CODA marker at the base are $0.30 \mathrm{~mm}$ and $0.96 \mathrm{~mm}$ in the $\mathrm{x}$ and $\mathrm{y}$
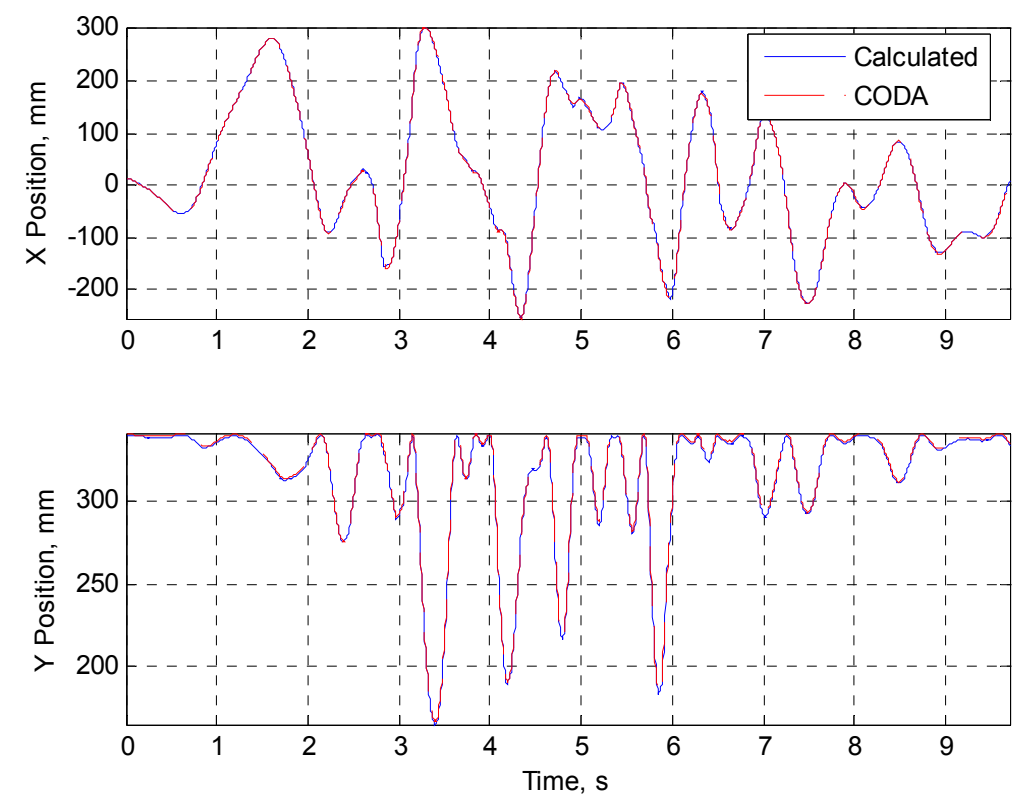

Figure 5.12. Base position in the $\mathrm{x}$ - and $\mathrm{y}$-directions for position 2 in test 1 . 
Table 5.3. Mean and standard deviations for base x-coordinate differences, millimeters.

\begin{tabular}{|c|c|c|c|c|c|c|c|c|}
\cline { 2 - 9 } \multicolumn{1}{c|}{} & \multicolumn{2}{c|}{ Test 1 } & \multicolumn{2}{c|}{ Test 2 } & \multicolumn{2}{c|}{ Test 3 } & \multicolumn{2}{c|}{ Test 4 } \\
\hline Marker & mean & $\sigma$ & mean & $\sigma$ & mean & $\sigma$ & mean & $\sigma$ \\
\hline 1 & -0.13 & 1.25 & -0.39 & 0.72 & -1.55 & 3.00 & -0.43 & 1.59 \\
\hline 2 & -0.30 & 1.38 & -0.29 & 0.93 & -1.53 & 1.88 & -0.31 & 0.87 \\
\hline 3 & 1.24 & 1.87 & 0.97 & 1.17 & 0.38 & 2.97 & 1.14 & 1.59 \\
\hline 7 & 1.50 & 2.68 & 1.37 & 1.20 & 0.54 & 3.22 & 1.46 & 1.63 \\
\hline 8 & -5.31 & 6.86 & 9.33 & 6.16 & -0.95 & 5.84 & 1.74 & 1.63 \\
\hline 9 & -0.37 & 2.04 & -0.12 & 1.06 & -1.64 & 1.51 & -0.21 & 0.57 \\
\hline
\end{tabular}

directions, respectfully. The total ranges of travel for both are $559.75 \mathrm{~mm}$ and $508.03 \mathrm{~mm}$ in the two directions. These discrepancies produce accuracies in the two directions of 99.95 percent and 99.81 percent, respectfully. The results for all markers and tests are in Tables 5.5 and 5.6.

\subsection{Conclusions}

Using the test stand representing the major components undergoing and influencing the motion of a body it was shown how accurately the coordinates of the underlying rigid body can be determined. The majority of the base positions calculated are over 99 percent accurate. More than a single global coordinate system was used to conclude the validity of this approach in reducing the effect of soft-tissue motion in rigid body estimation. Drift was shown to be a significant source of error in the analysis of position based on accelerometer data. Recalibrating the data with CODA measurements would limit the accuracy of the accelerometer data to that of

Table 5.4. Mean and standard deviations for base y-coordinate differences, millimeters.

\begin{tabular}{|c|c|c|c|c|c|c|c|c|}
\cline { 2 - 9 } \multicolumn{1}{c|}{} & \multicolumn{2}{c|}{ Test 1 } & \multicolumn{2}{c|}{ Test 2 } & \multicolumn{2}{c|}{ Test 3 } & \multicolumn{2}{c|}{ Test 4 } \\
\hline Marker & mean & $\sigma$ & mean & $\sigma$ & mean & $\sigma$ & mean & $\sigma$ \\
\hline 1 & 0.97 & 0.53 & 1.80 & 0.23 & 2.65 & 1.46 & -1.09 & 0.52 \\
\hline 2 & 0.96 & 0.64 & 1.83 & 0.33 & 2.75 & 0.68 & -1.30 & 0.22 \\
\hline 3 & -0.65 & 0.74 & -1.37 & 0.36 & -2.48 & 1.30 & 1.41 & 0.42 \\
\hline 7 & 1.10 & 2.34 & 1.51 & 0.86 & 1.88 & 1.94 & 0.45 & 0.76 \\
\hline 8 & 0.95 & 5.53 & 1.35 & 6.61 & 1.96 & 2.90 & 0.08 & 0.40 \\
\hline 9 & 3.99 & 2.38 & 4.03 & 1.54 & 4.45 & 1.53 & 2.63 & 0.51 \\
\hline
\end{tabular}


Table 5.5. Base $\mathrm{x}$-axis range and accuracy of calculated values to CODA measurements.

\begin{tabular}{|c|c|c|c|c|c|c|c|c|}
\cline { 2 - 9 } \multicolumn{1}{c|}{} & \multicolumn{2}{c|}{ Test 1 } & \multicolumn{2}{c|}{ Test 2 } & \multicolumn{2}{c|}{ Test 3 } & \multicolumn{2}{c|}{ Test 4 } \\
\hline Marker & trav., mm & acc., \% & trav., mm & acc., \% & trav., mm & acc., \% & trav., mm & acc., \% \\
\hline 1 & 345.03 & 99.96 & 360.34 & 99.89 & 266.43 & 99.42 & 297.09 & 99.86 \\
\hline 2 & 559.75 & 99.95 & 627.07 & 99.95 & 333.48 & 99.54 & 289.18 & 99.89 \\
\hline 3 & 438.49 & 99.72 & 495.15 & 99.80 & 292.20 & 99.87 & 262.77 & 99.57 \\
\hline 7 & 681.47 & 99.78 & 837.20 & 99.84 & 316.02 & 99.83 & 339.61 & 99.57 \\
\hline 8 & 916.54 & 99.42 & 1152.94 & 99.19 & 479.66 & 99.80 & 523.29 & 99.67 \\
\hline 9 & 702.38 & 99.95 & 997.12 & 99.99 & 372.62 & 99.56 & 419.37 & 99.95 \\
\hline
\end{tabular}

CODA. Thus CODA measurements were used directly for position measurements. From these results, it can be assumed that larger, three-dimensional studies can be undertaken with results similar to the ones already shown which can further aid in vivo studies.

Table 5.6. Base y-axis range and accuracy of calculated values to CODA measurements.

\begin{tabular}{|c|c|c|c|c|c|c|c|c|}
\cline { 2 - 9 } \multicolumn{1}{c|}{} & \multicolumn{2}{c|}{ Test 1 } & \multicolumn{2}{c|}{ Test 2 } & \multicolumn{2}{c|}{ Test 3 } & \multicolumn{2}{c|}{ Test 4 } \\
\hline Marker & trav., mm & acc., \% & trav., mm & acc., \% & trav., mm & acc., \% & trav., mm & acc., \% \\
\hline 1 & 165.56 & 99.42 & 187.35 & 99.04 & 189.35 & 98.60 & 200.39 & 99.45 \\
\hline 2 & 508.03 & 99.81 & 571.80 & 99.68 & 590.06 & 99.53 & 595.93 & 99.78 \\
\hline 3 & 333.10 & 99.81 & 384.35 & 99.64 & 371.35 & 99.33 & 389.04 & 99.64 \\
\hline 7 & 913.30 & 99.88 & 998.99 & 99.85 & 1001.46 & 99.81 & 1004.04 & 99.96 \\
\hline 8 & 1186.73 & 99.92 & 1222.76 & 99.89 & 1358.91 & 99.86 & 1356.56 & 99.99 \\
\hline 9 & 1107.78 & 99.64 & 1140.63 & 99.65 & 1215.52 & 99.63 & 1199.24 & 99.78 \\
\hline
\end{tabular}




\section{Chapter 6. Conclusions and Recommendations}

\subsection{Experimental Conclusions}

\subsubsection{Use of Multiple Data Streams in Determining Position}

Measuring a specific variable over time requires a sensor properly designed to accomplish the task. For many tasks, such as motion studies, both precision and accuracy are necessary to understand the exact path of a point on the body during motion. However, regardless of how well a particular sensor is designed, its characteristic weaknesses may be exploited during its use. Using a combination of sensors and combining them with a Kalman filter was determined necessary to not only compensate for the weaknesses of one sensor with another of a different type, but also to increase the likelihood of accurate measurements by decreasing their uncertainty.

The unfortunate weakness of inexpensive accelerometers in determining position is their tendency to drift over extended periods of time. Without implementing recalibration of this data against stable position and/or velocity sources during the integration process, its use will be scrutinized for any test requiring high accuracy. For this reason, and the fact that recalibrating accelerometer data using CODA markers at the same locations will yield data that cannot be any more accurate than the CODA position data itself, position integrated from acceleration data was not considered for the Kalman filter and direct position measurements were assumed the filtered output.

\subsubsection{Using Accelerometers to Remove the Influence of Soft Tissue Motion}

The use of accelerometers in this experiment showed the potential of removing the influence of soft tissue motion in current motion studies. Using direct acceleration data, with the knowledge of system masses and spring constants, values for soft tissue artifact were determined. 
Plotting these values over time with those found from direct position measurements using the CODA markers showed close agreements in the mass motions. Calculating the differences of these two graphs and taking the mean values of each shows exactly how good the agreements are between the two. Not including the data of mass number 8 , which experienced mechanical problems, the mean differences between the accelerometer data and CODA data were less than 1 $\mathrm{mm}$ with the largest across all tests being $0.66 \mathrm{~mm}$.

Calculating the position of the underlying bone in this experiment required knowing the initial separation between the bases and sprung masses. This was determined using the initial separation distance between the two CODA markers at each location since the sprung CODA marker was placed on top of the accelerometer and the base value made a reference point for each system to be compared. With all variables known, the appropriate calculations were made to compare the global $\mathrm{x}$ and $\mathrm{y}$ base coordinate differences between the CODA and accelerometer systems. Considering the total global horizontal and vertical displacements of the base positions, all accelerometer $\mathrm{x}$ positions were calculated within $2 \mathrm{~mm}$ of the CODA measurements, and the y values were within $4 \mathrm{~mm}$ with the largest deviations of $1.64 \mathrm{~mm}$ and $4.45 \mathrm{~mm}$, respectively. Both of these values exclude marker number 8 and the largest deviations occur for marker number 9. The recorded accuracies at this marker are 99.56 percent in the $\mathrm{x}$ direction and 99.63 percent for the $y$ direction over the displacement of the base in each direction.

\subsubsection{Conclusions Regarding Acceleration Data and Determination of Bone Data}

The process of determining the position of the bone over time using accelerometers was determined to be accurate only if another type of sensor was also included, in this instance, a position sensor. The effect of drift was too influential on the determination of position to be included in tissue surface location, but the acceleration data itself was found to be helpful in finding the motion of the tissue relative to the bone itself. 


\subsection{Recommendations for Future Work}

Using this approach as a method to find the position of a link, using a mass with one degree of freedom, shows potential for future endeavors. One recommendation is to expand the degrees of freedom of the system into one considering three-dimensional motion of the mass. This would allow for a more realistic interpretation of living organisms and actual motion produced. This variation would only affect the additional natural frequencies needed for determination of three-dimensional artifact values. With consideration of these new values, a three-dimensional interpretation of base motion would be needed where this experiment only covers two dimensions. Even so, the addition of dimensional movement should still exhibit the properties of each dimension displayed in the current work. Knowing the visco-elastic properties of the system should provide for acceptable results of three-dimensional data.

In order to obtain results similar to those displayed in this research for living organisms, the visco-elastic variables of the tissue must be known for the organism under study. One method available to explore these properties is to include the use of electromyography (EMG) data at various, scattered locations along the members to be analyzed. For varying values of recorded muscle flexion, the mechanical resistance and dampening of the tissue can be determined using the electrical resistance found in the process.

An addition of different types, or different numbers, of sensors could be incorporated to limit the effect of drift for the accelerometers. For MEMS applicability, angular rate sensors, or gyroscopes, can be implemented for such a task. This can be done separate from the accelerometers or as part of the accelerometer to make an inertial measurement unit (IMU). This unit is used in the aviation industry to measure the position and motion of an aircraft over time. 
In clinical practice or for any tests performed on biological subjects, the actual threedimensional coordinates of the underlying bones will not be given, and neither will it be for the joints taking part in the motions. Furthermore, the activities of the joint, be it two or three dimensional, will need to be determined over the motions. This will give a greater understanding of how complex joints such as the knee perform given an exercise.

By modeling the limb under analysis mathematically, using acquired marker data to resolve a rigid body, such joint activity can be explored. One method was introduced by Schut $[1960 ; 1967]$ that uses three or more non-collinear points on a body and the coordinates of each before and after translation and rotation. The only restriction is that the rotation cannot be $180^{\circ}$ or even near that value [Schut 1960 and 1967]. The methods proposed draw on the use of simple formulas and were published with a FORTRAN program that could be used for a study once base coordinates have been determined using methods such as the one detailed in this thesis. 


\section{References}

Alexander, E., Andriacchi, T. (2000) Correcting for deformation in skin-based marker systems. Journal of Biomechanics. 34: 355-361.

Andriacchi, T., Alexander, E., Toney, M., Dyrby, C., and Sum J. (1998) A point cluster method for in vivo motion analysis: Applied to a study of knee kinematics. Journal of Biomechanical Engineering. 120: 743-749.

Back, W., Schamhardt, H.C., Savelberg, H.H.C.M., van den Bogert, A.J., Bruin, G., Hartman, W., and Barneveld, A. (1995) How the horse moves: 1. Significance of graphical representations of equine forelimb kinematics. Equine Veterinary Journal. 27: 31-38.

Back, W., Schamhardt, H.C., Savelberg, H.H.C.M., van den Bogert, A.J., Bruin, G., Hartman, W., and Barneveld, A. (1995) How the horse moves: 2. Significance of graphical representations of equine hind limb kinematics. Equine Veterinary Journal. 27: 39-45.

Barrance, P., Williams, G., Novotny, J., and Buchanan, T. (2005) A method for measurement of joint kinematics in vivo by registration of 3-D geometric models with cine phase contrast magnetic resonance imaging data. Journal of Biomechanical Engineering. 127: 829-837.

Beer, Ferdinand P. and Johnson, E. Russel Jr. Vector Mechanics for Engineers: Statics, $6^{\text {th }}$ ed. New York: McGraw Hill, 1996.

Cappello, A., Cappozzo, A., Palombara, P., Lucchetti, L., and Leardini, A. (1997) Multiple anatomical landmark calibration for optimal bone pose estimation. Human Movement Science. 16: $259-274$.

Cappozzo, A., Catani, F., Della Croce, U, and Leardini, A. (1995) Position and orientation in space of bones during movement: anatomical frame definition and determination. Clinical Biomechanics. 10: 171-178.

Cappozzo, A., Catani, F., Leardini, A., Benedetti, M. G., and Della Croce, U. (1996) Position and orientation in space of bones during movement: experimental artefacts. Clinical Biomechanics. 11: 90-100.

Cappozzo, A., Della Croce, U., Leardini, A., and Chiari, L. (2005) Human movement analysis using stereophotogrammetry. Part 1: theoretical background. Gait and Posture. 21: 186-196.

Chiari, L., Della Croce, U., Leardini, A., and Cappozzo, A. (2005) Human movement analysis using stereophotogrammetry. Part 2: Instrumental errors. Gait and Posture. 21: 197-211.

Clayton, H., and Schamhardt, H., (2000) Equine Locomotion: Chapter 3, Measurement Techniques for Gait Analysis. in Back, W., and Clayton, H. Equine Locomotion. London: Saunders, 2000. 
Fuller, J., Liu, L.-J., Murphy, M.C., and Mann, R.W. (1997) A comparison of lower-extremity skeletal kinematics measured using skin- and pin-mounted markers. Human Movement Science. 16: $219-242$.

Fuller, John E. "Development and characterization of velocity workspaces for the human knee." Ph.D. dissertation, Louisiana State University, 2001.

Fung, Y. Biomechanics: Mechanical properties of living tissues. New York: Springer-Verlag, 1981.

Gray, H. Gray's Anatomy. $38^{\text {th }}$ ed. Williams P, Bannister L, Berry M, Collins P, Dyson M, Dussek J, Ferguson M, editors. New York: Churchill Livingstone, 1995. p. 2092.

Gruen, Armin. (1997) Fundamentals of videogrammetry - A review. Human Movement Science. 16: $155-187$.

Karduna, A., McClure, P., Michener, L., and Sennett, B. (2001) Dynamic measurements of three-dimensional scapular kinematics: a validation study. Journal of Biomechanical Engineering. 123: 184-190.

Lafortune, M. (1991) Three-dimensional acceleration of the tibia during walking and running. Journal of Biomechanics. 24: 877-886.

Lafortune, M.A., Cavanagh, P.R., Sommer, H.J., and Kalenak, A. (1992) Three dimensional kinematics of the human knee during walking, Journal of Biomechanics, 25: 347-357.

Leach, B. W., and MacPherson, J. I. (1991) An application of Kalman filtering to airborne wind measurement. Journal of Atmospheric and Oceanic Technology. 8: 51-65.

Lerner, A., Tamez-Pena, J., Houck, J., Yao, J., Harmon, H., Salo, A., and Totterman, S. (2003) The use of sequential MR image sets for determining tibiofemoral motion: reliability of coordinate systems and accuracy of motion tracking algorithm. Journal of Biomechanical Engineering. 125: 246-253.

Lucchetti, L., Cappozzo, A., Cappello, A., and Della Croce, U. (1998) Skin movement artifact assessment and compensation in the estimation of knee-joint kinematics. Journal of Biomechanics. 31: 977-984.

Luinge, H. J. “Inertial sensing of human movement.” Ph.D. dissertation, University of Twente, 2002.

Maybeck, Peter S. Stochastic models, estimation and control. New York: Academic Press, 1979.

Murphy, M. C. "Geometry and the kinematics of the normal human knee." Ph.D. dissertation, Massachusetts Institute of Technology, Cambridge, 1990. 
Muybridge, Eadweard. Animals in Motion. New York: Dover Publications, 1899.

Nigg, B. and Herzog, W. Biomechanics of the musculo-skeletal system, $2^{\text {nd }}$ ed. New York: John Wiley and Sons, Inc., 1999.

Oxford English Dictionary, 2006, http://www.oed.com.

Pang, G., and Liu, H. (2001) Evaluation of a low-cost MEMS accelerometer for distance measurement. Journal of Intelligent and Robotic Systems. 30: 249-265.

Sakaguchi, T., Kanamori, T., Katayose, H., Sato, K., and Inokuchi, S. Human motion capture by integrating gyroscopes and accelerometers. Proceedings of the 1996 IEEE/SICE/RSJ International Conference on Multisensor Fusion and Integration for Intelligent Systems, 1996, pp. $470-475$.

Schut, G. H . (1960) On exact linear equations for the computation of the rotational elements of absolute orientation. Photogrammetria, 17: 34-37.

Schut, G. H., (1967) "Formation of Strips from Independent Models", AP-PR 36, NRC-9695, Division of Applied Physics, National Research Council of Canada, Ottawa.

Stagni, R., Fantozzi, S., Cappello, A., and Leardini, A. (2003) Validation of the Interval Deformation Technique for Compensating Soft Tissue Artefact in Human Motion Analysis. Lecture Notes in Computer Science. 2673: 293 - 301.

Tashman, S. and Anderst, W. (2003) In-vivo measurement of dynamic joint motion using high speed biplane radiography and CT: application to canine ACL deficiency. Journal of Biomechanical Engineering. 125: 238-245.

Taylor, W., Ehrig, R., Duda, G., Schell, H., Seebeck, P., and Heller, M. (2005) On the influence of soft tissue coverage in the determination of bone kinematics using skin markers. Journal of Orthopaedic Research. 23: 726-734.

Trujillo, D.M. and Busby, H.R. (1990) A mathematical method for the measurement of bone motion with skin-mounted accelerometers. Journal of Biomechanical Engineering. 112: 229231.

van Weeren, P.R. (2002) The clinical applicability of automated gait analysis systems. Equine Veterinary Journal. 34: 218-219.

Waldron, Kenneth J. and Kinzel, Gary L. Kinematics, Dynamics, and Design of Machinery. New York: John Wiley and Sons, Inc., 1999.

Welch, G. and Bishop, G. An Introduction to the Kalman Filter. Course 8, SIGGRAPH 2001. 
Weishaupt, M.A., Wiestner, T., Hogg, H.P., Jordan, P., Auer, J.A., and Barrey, E. (2001) Assessment of gait irregularities in the horse: eye vs. gait analysis. Equine Veterinary Journal, Supplement. 33: 135-140.

Wood, R., Stromberg, I. M., Jonas, P. R., and Mill, C. S. (1997) Analysis of an air motion system on a light aircraft for boundary layer research. Journal of Atmospheric and Oceanic Technology. 14: $960-968$. 


\section{Appendix A}

\section{Artifact Plots for All Six Marker Locations over Four Tests}
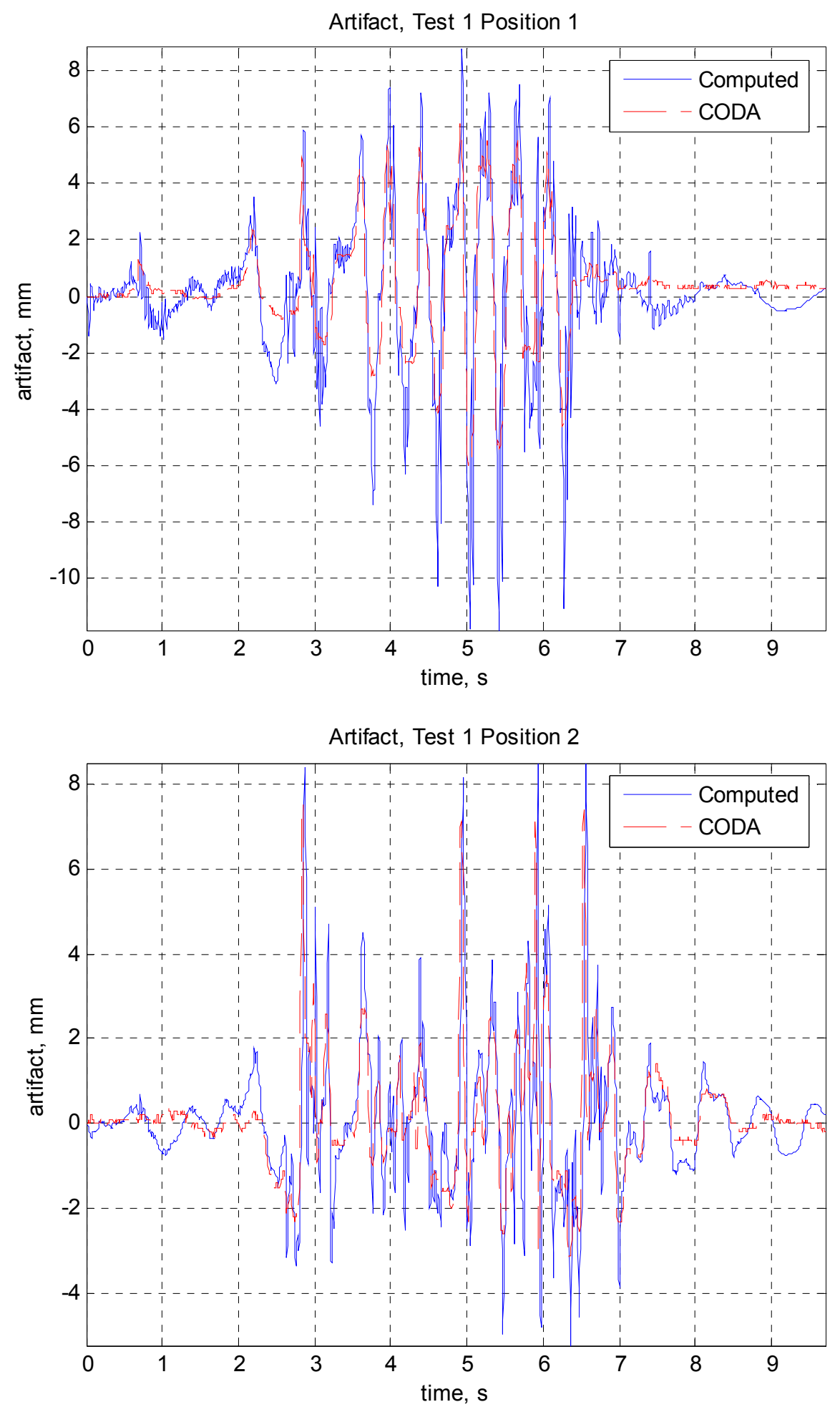
Artifact, Test 1 Position 3
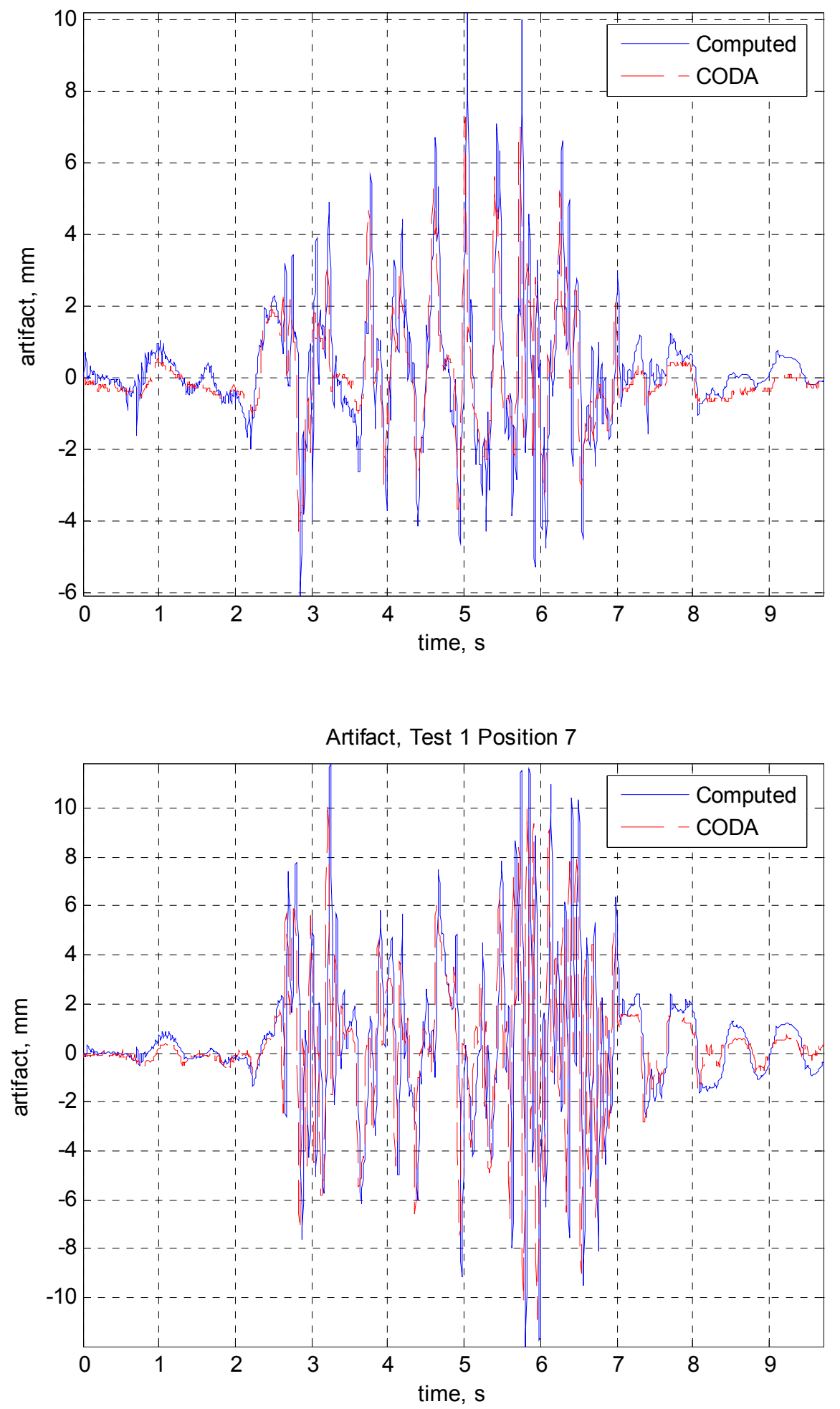

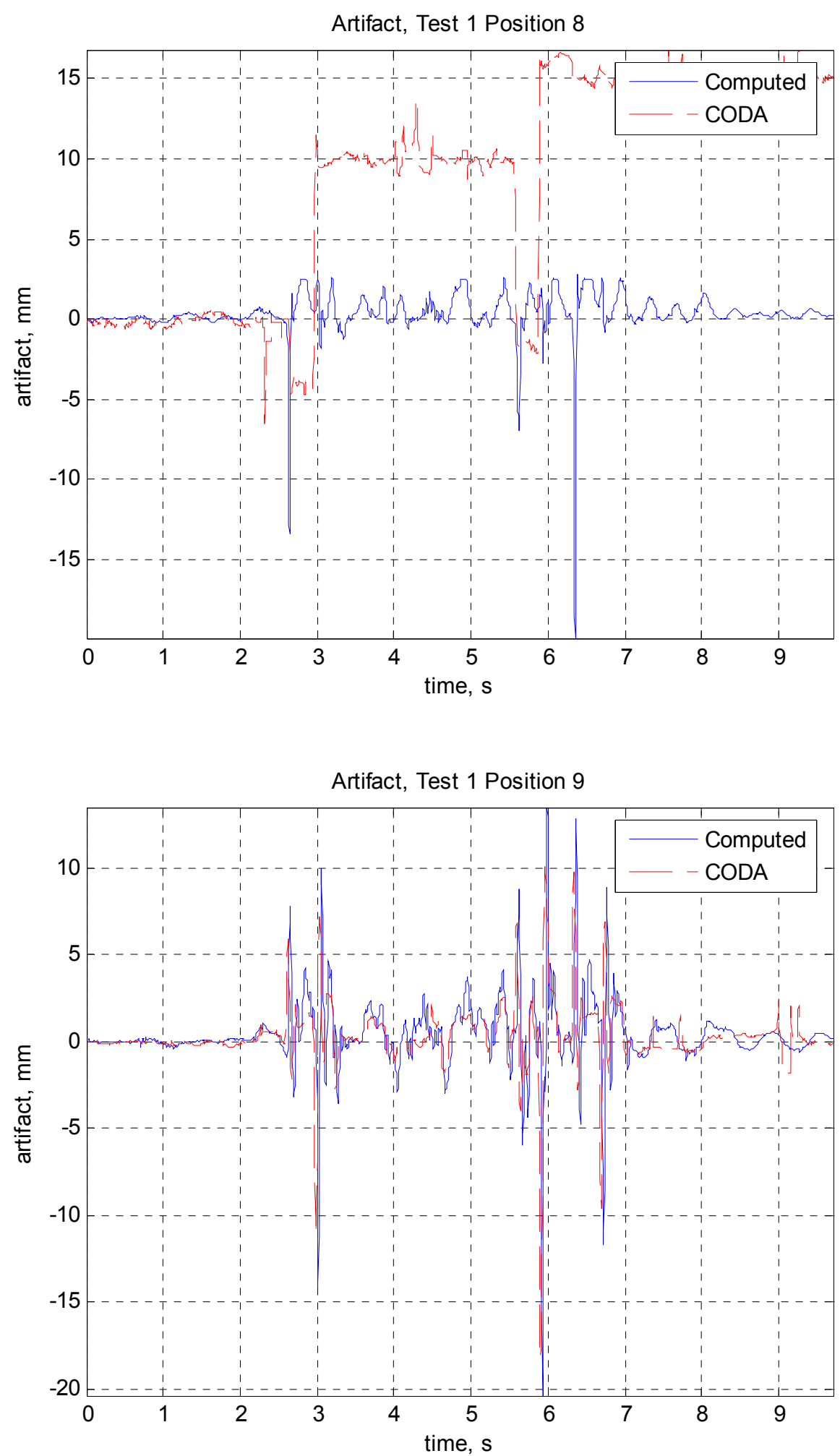



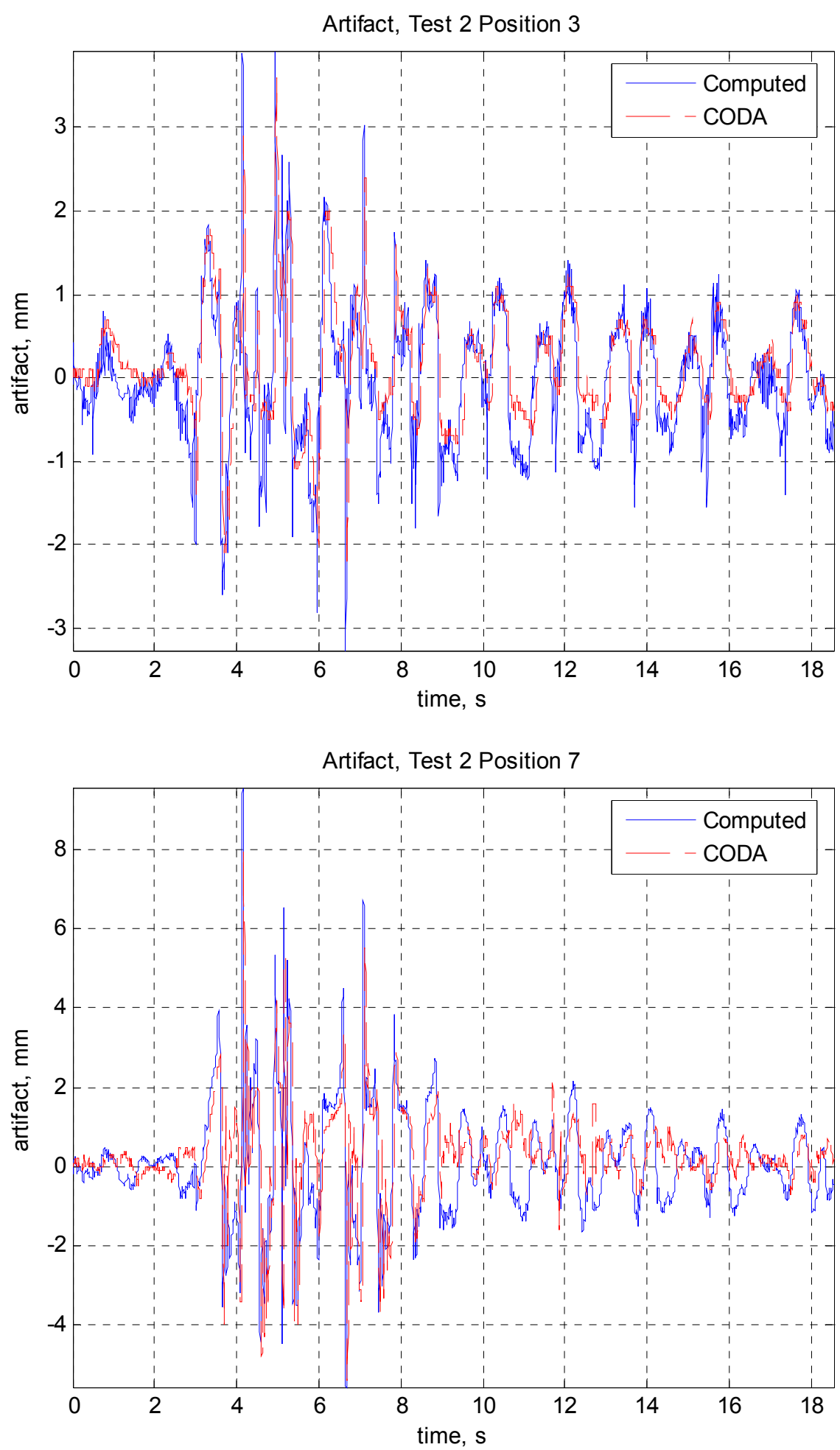

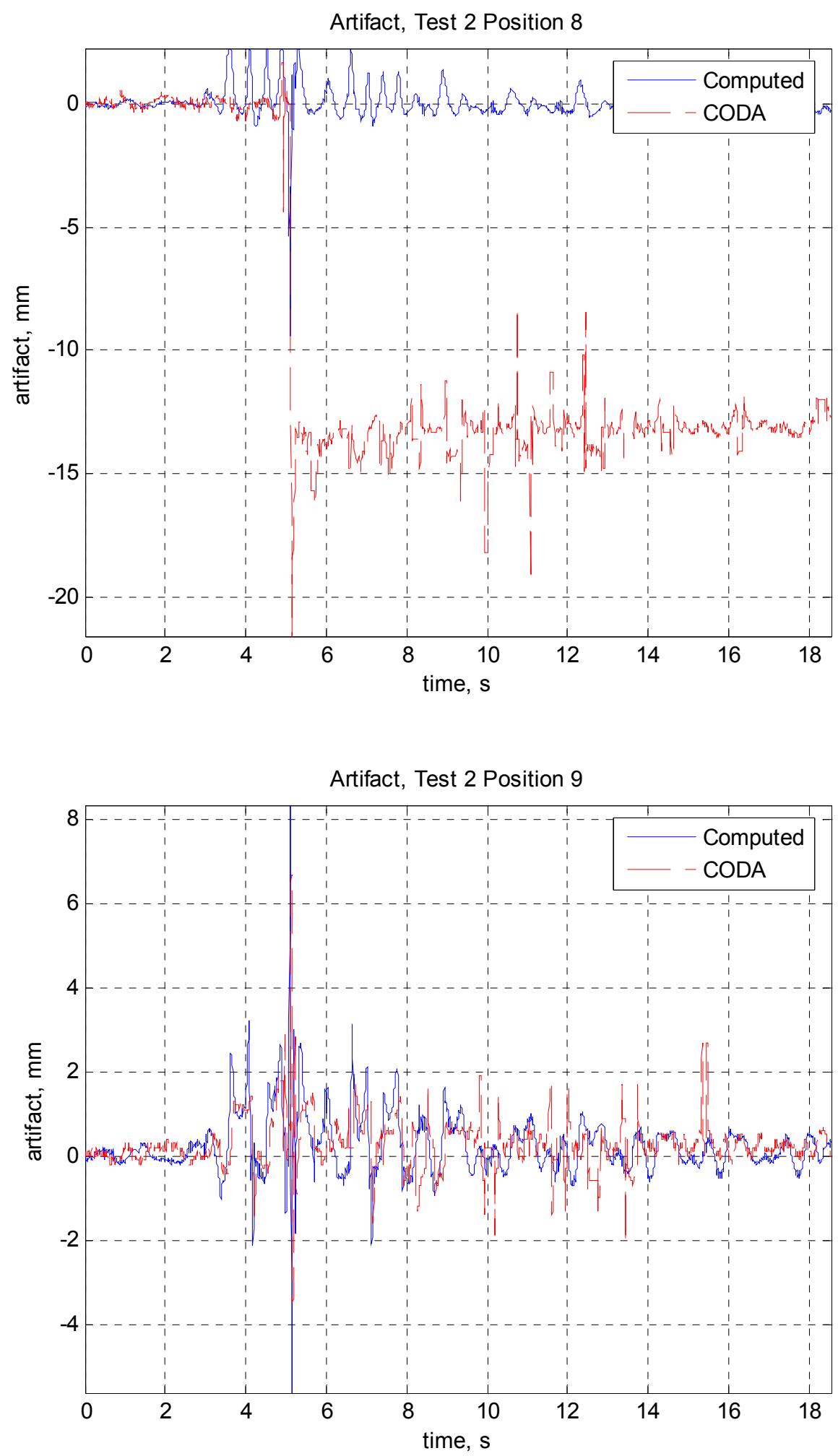

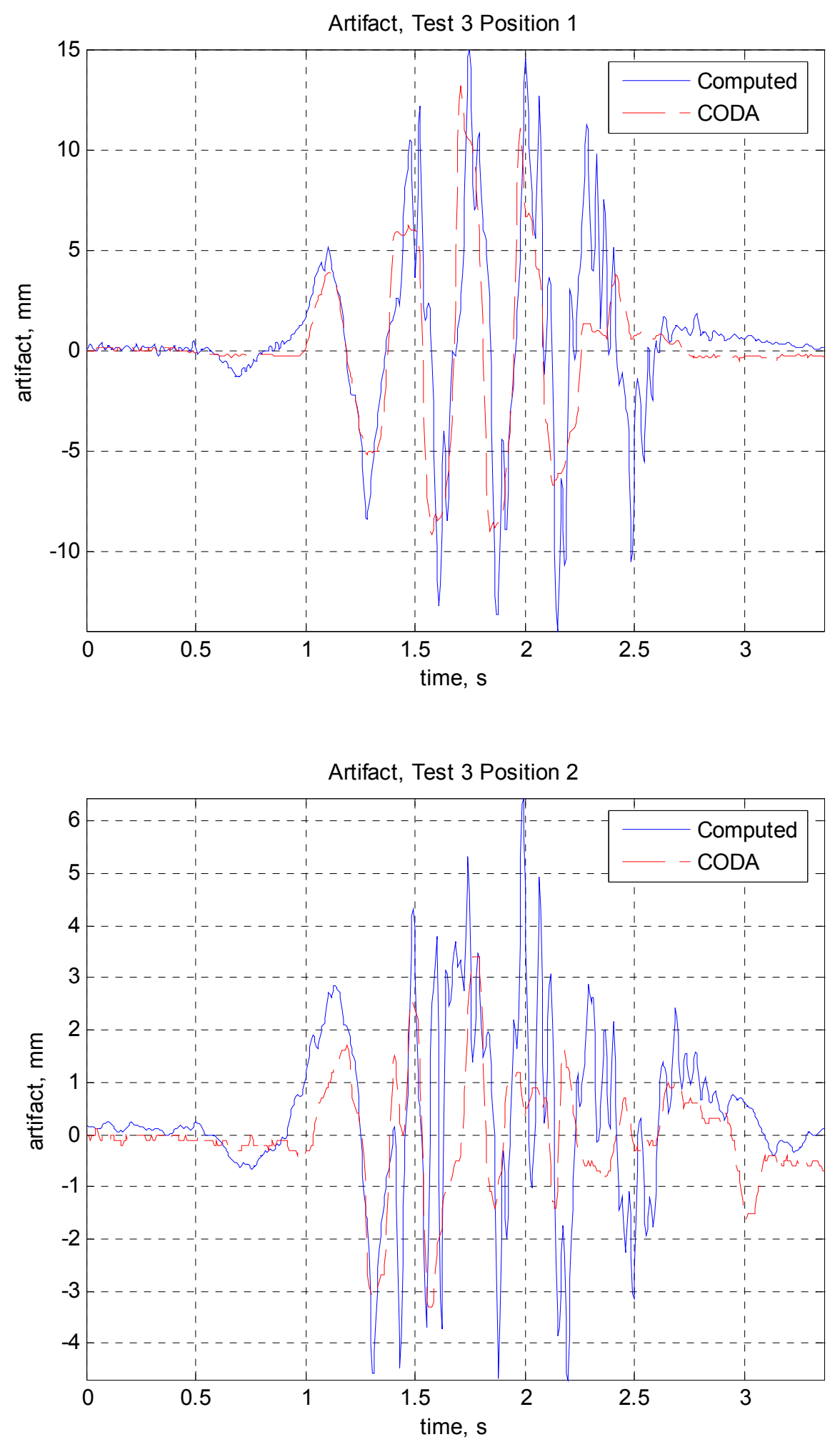

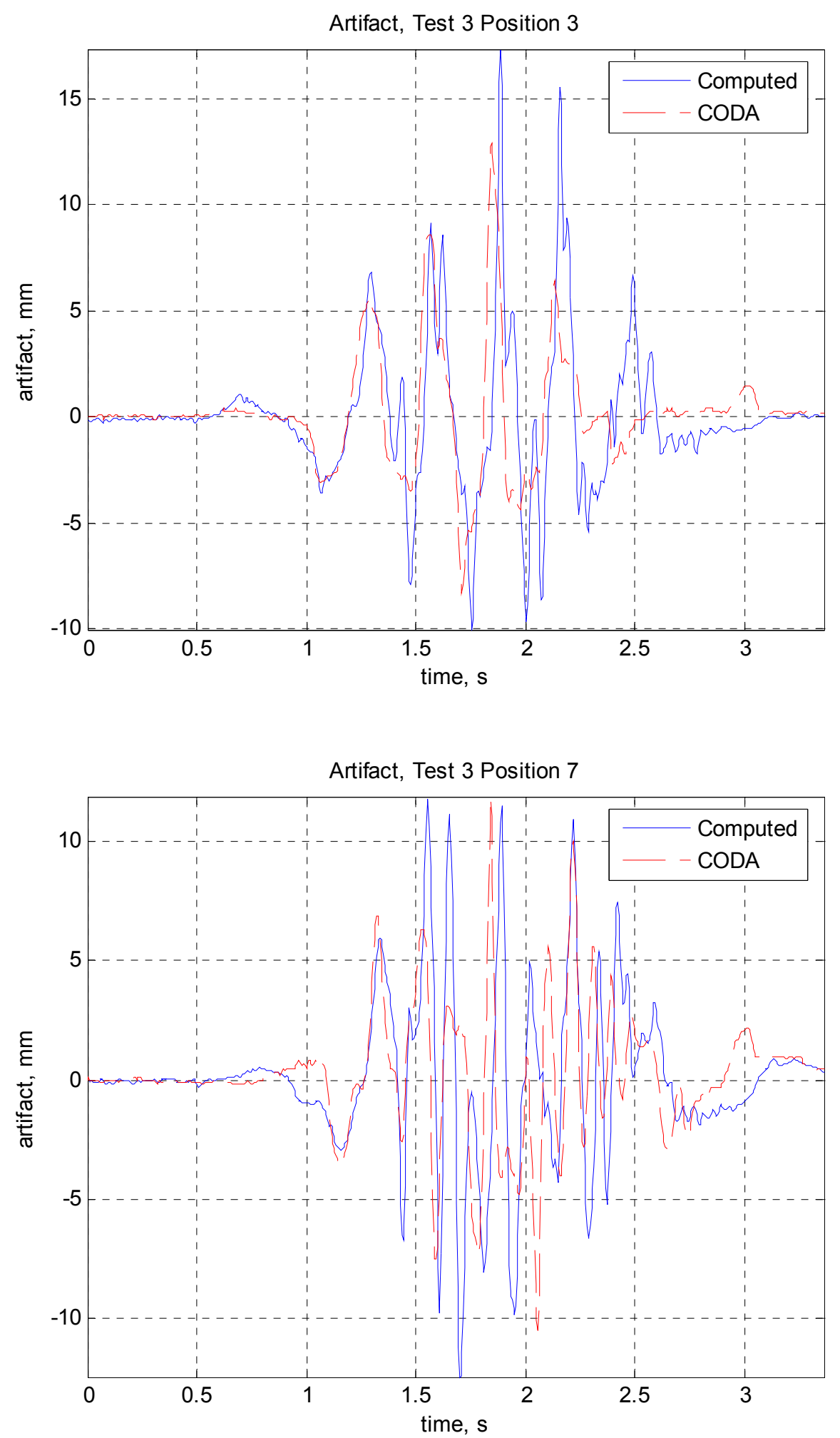

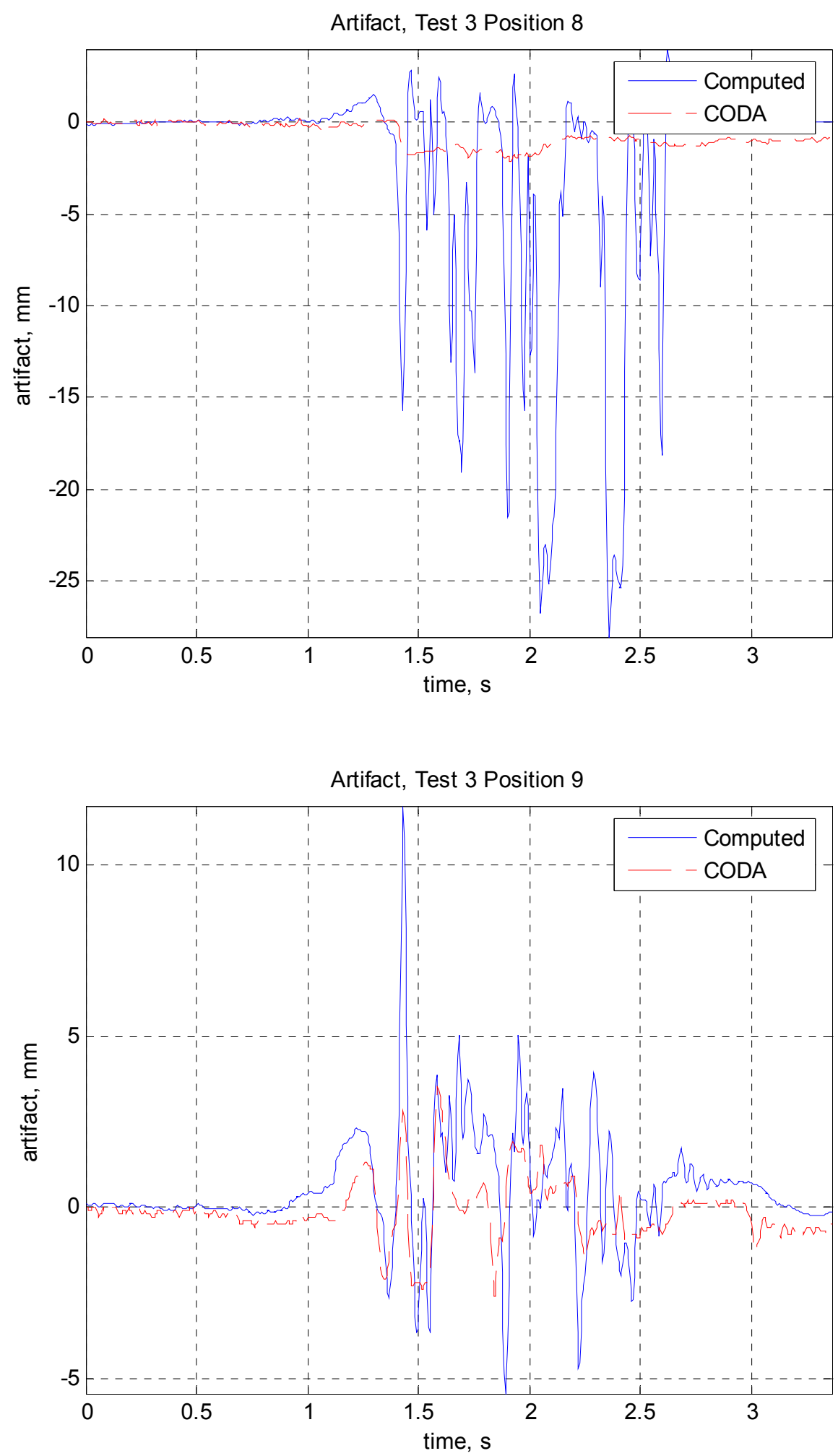

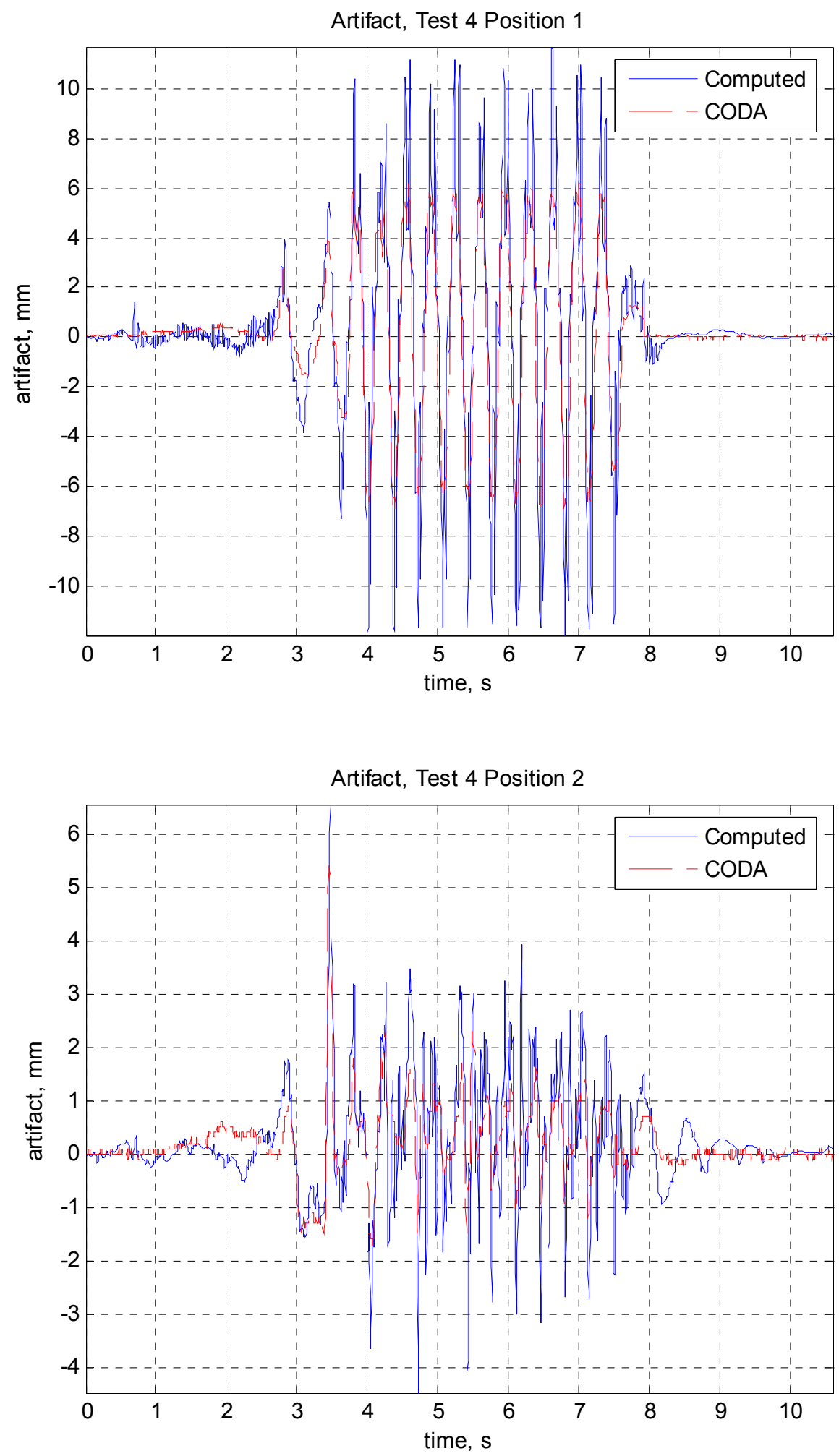

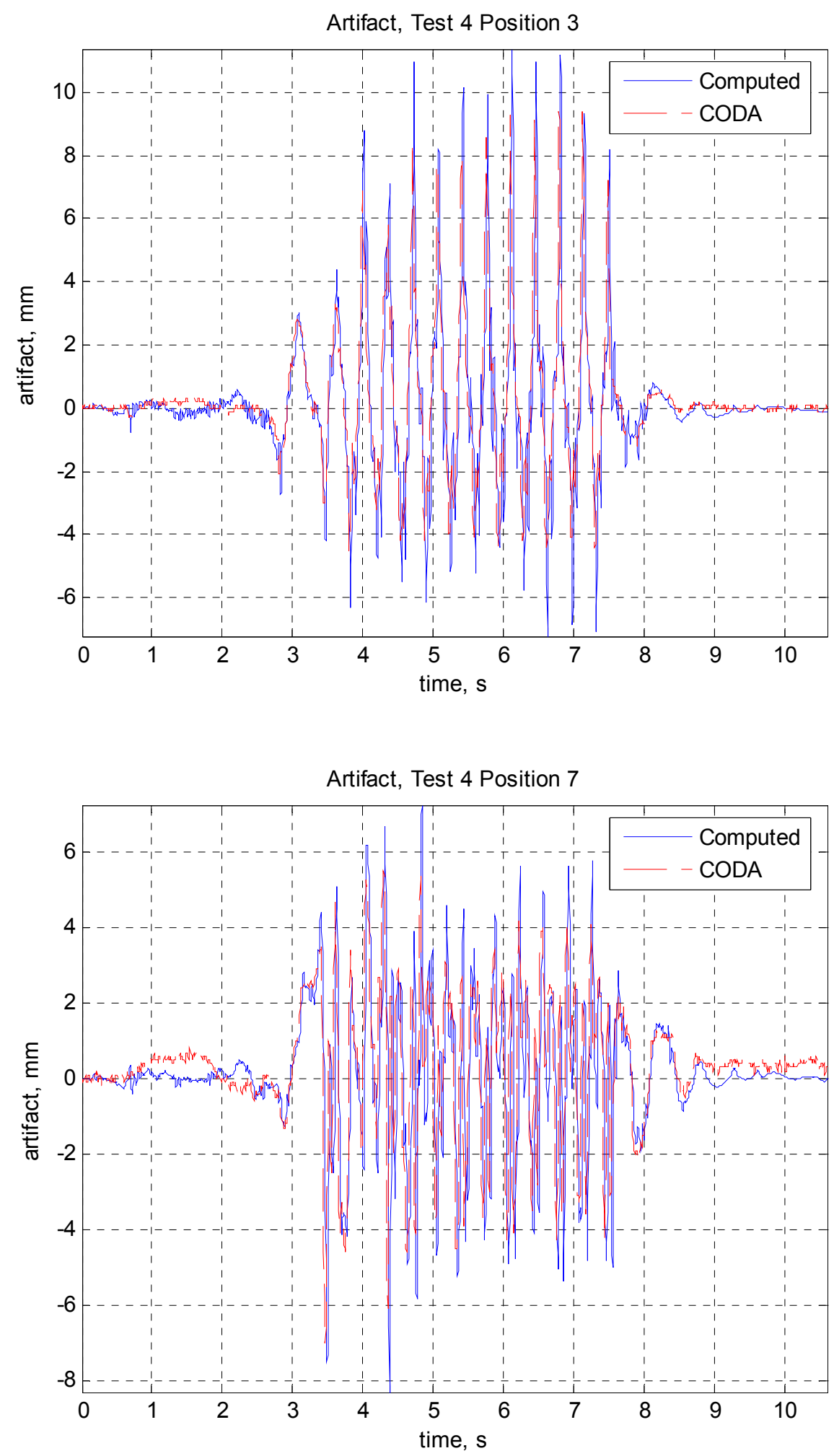

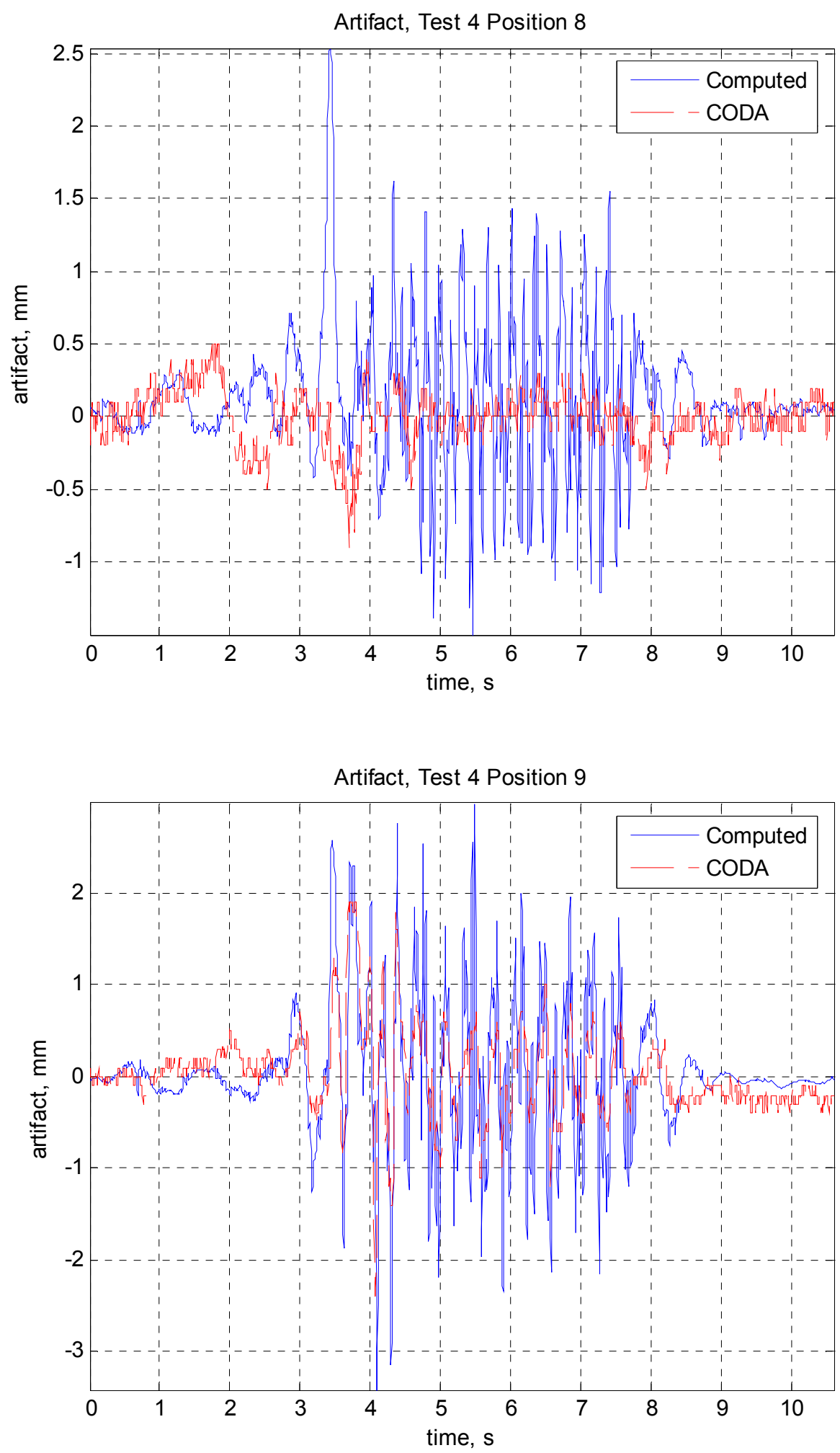


\section{Appendix B}

\section{Base Coordinate Comparisons between Two Sensor Sets}
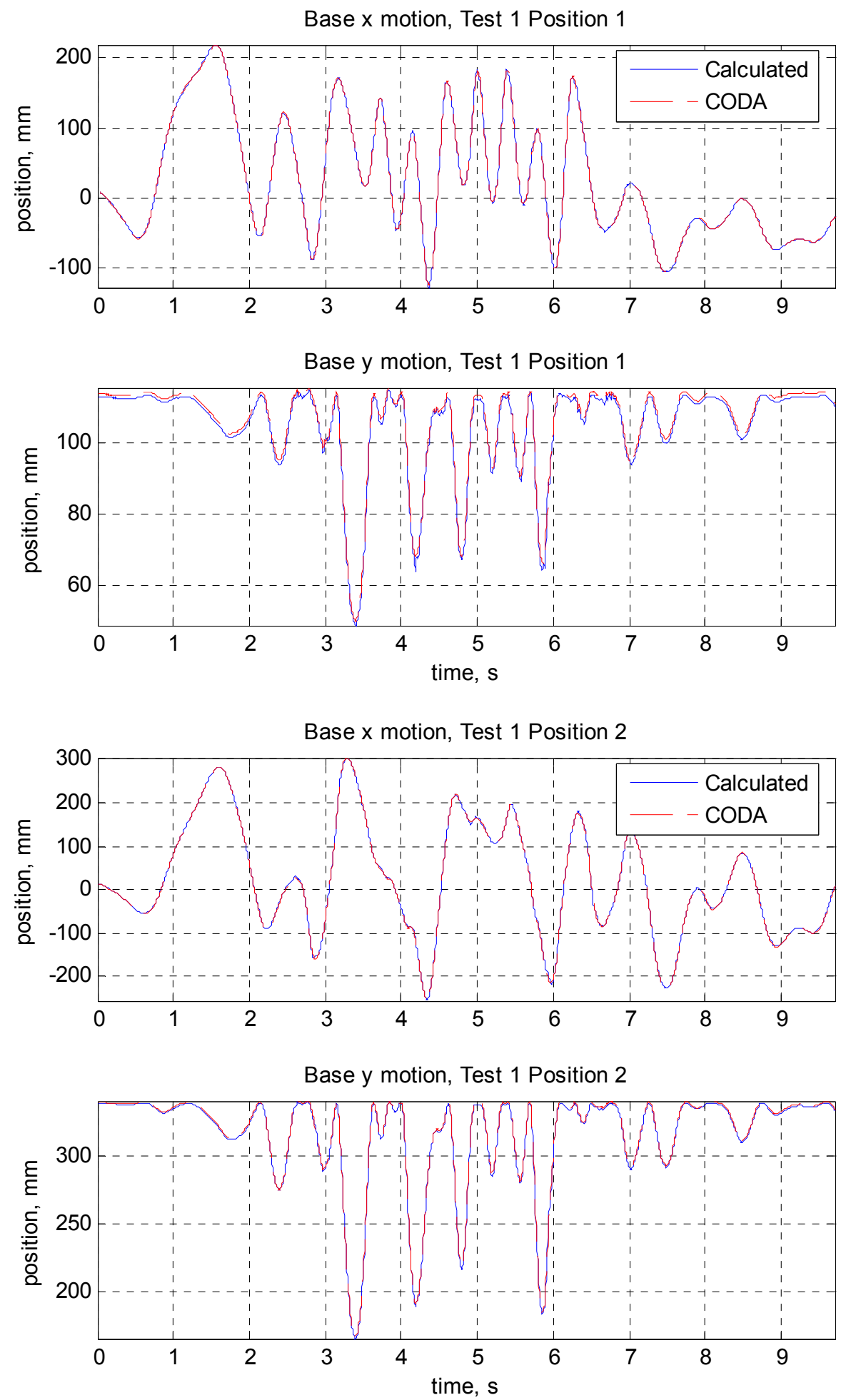
Base $\mathrm{x}$ motion, Test 1 Position 3

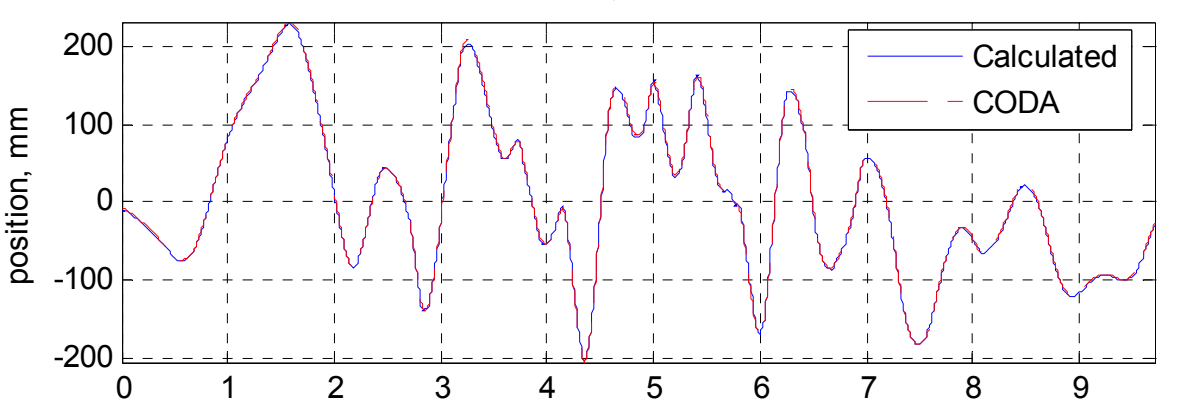

Base y motion, Test 1 Position 3
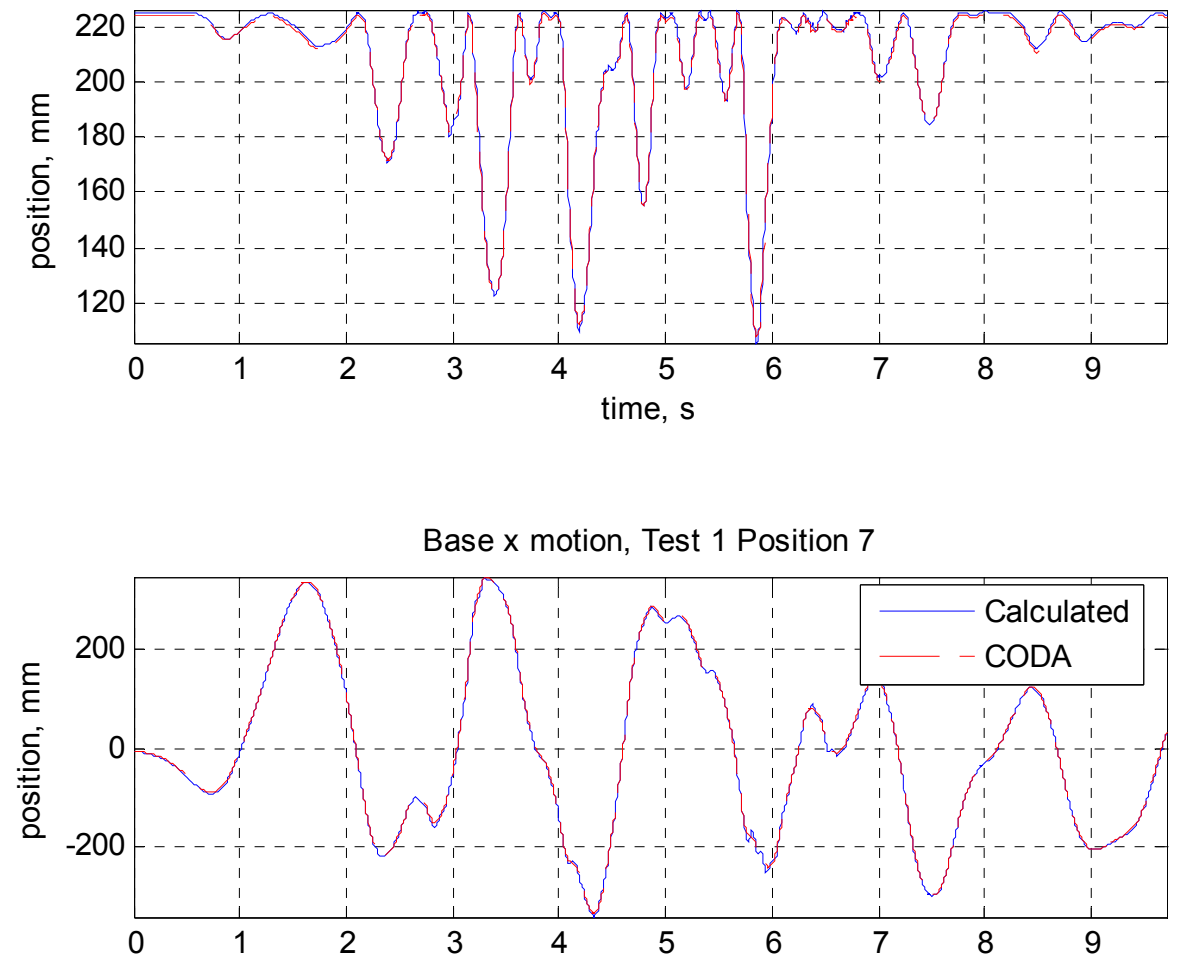

Base y motion, Test 1 Position 7

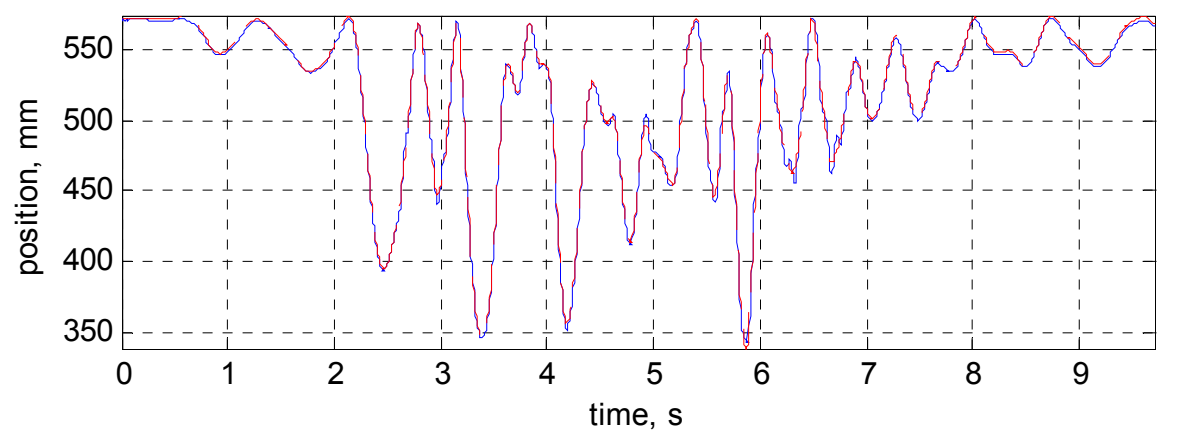


Base $\mathrm{x}$ motion, Test 1 Position 8

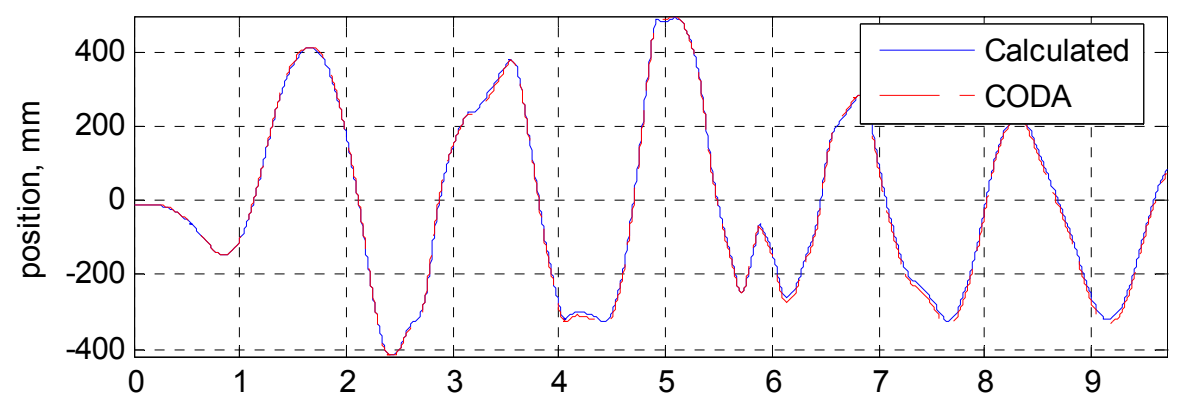

Base y motion, Test 1 Position 8
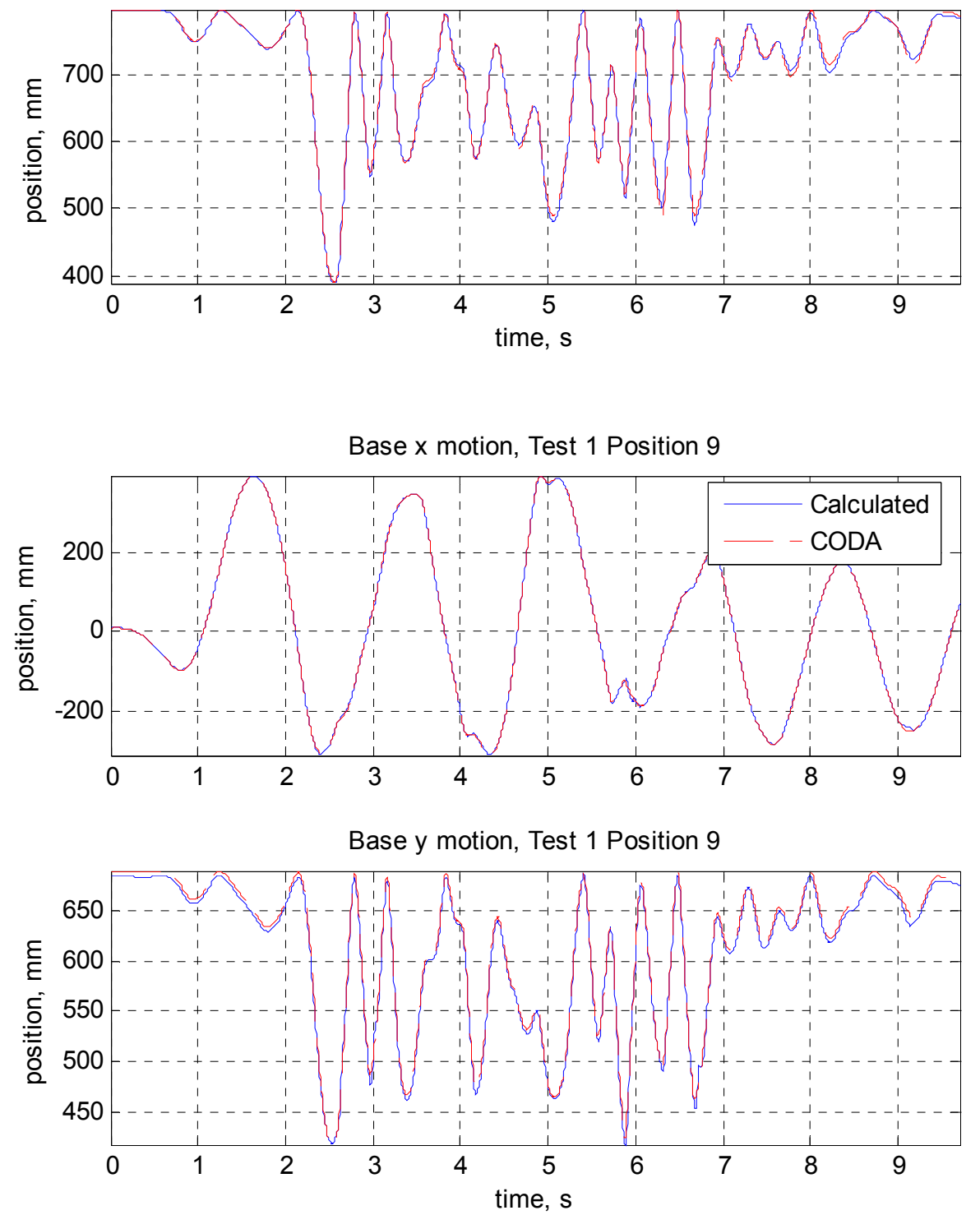
Base $\mathrm{x}$ motion, Test 2 Position 1

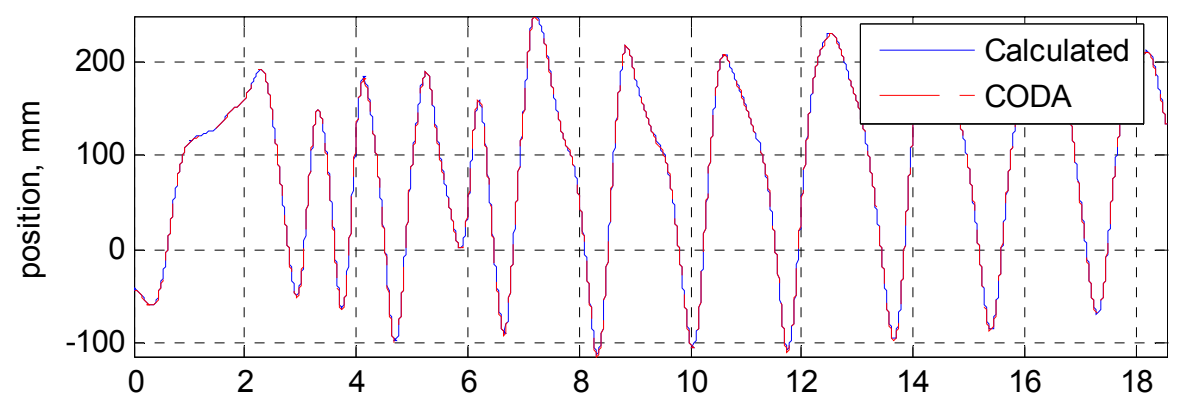

Base y motion, Test 2 Position 1
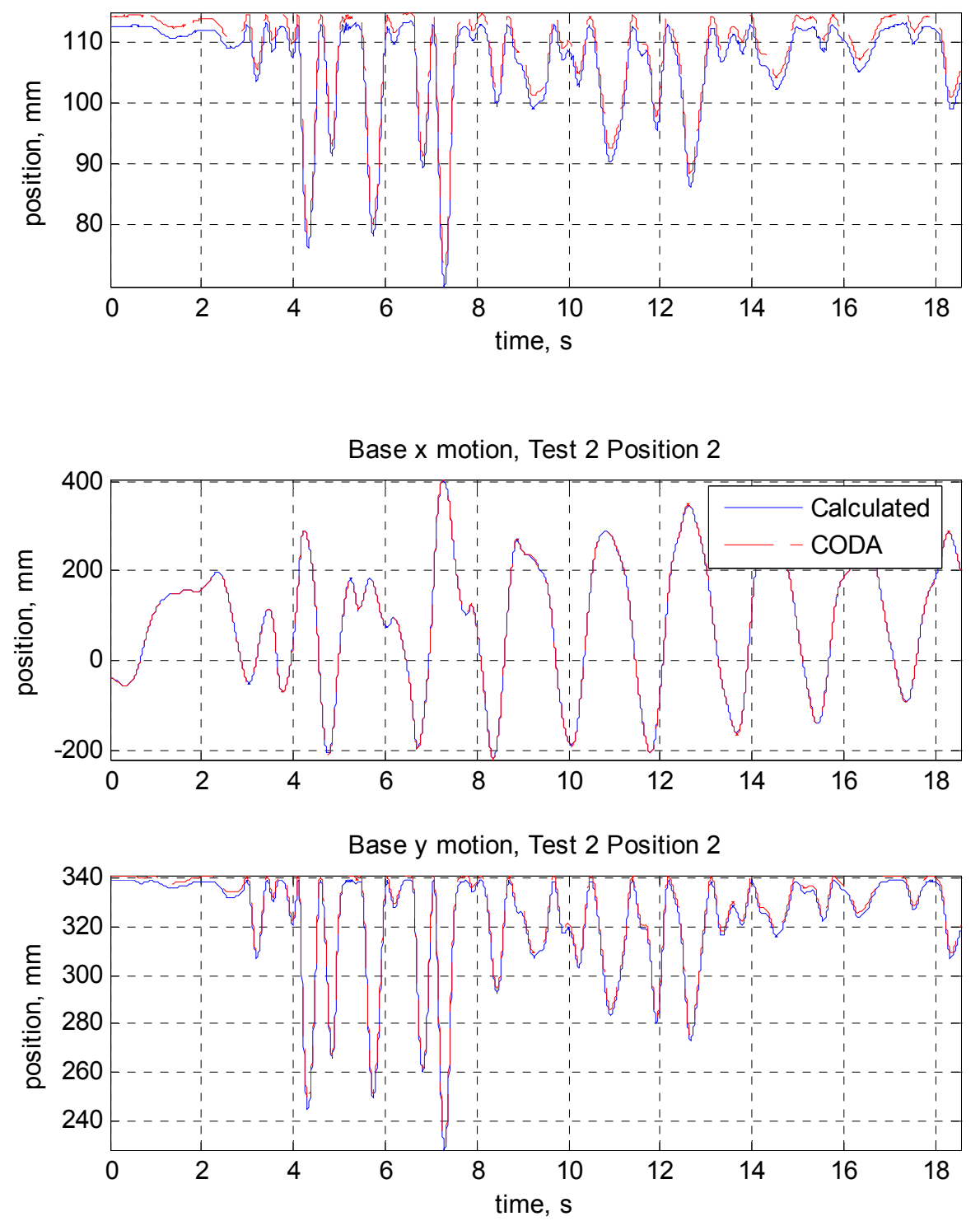
Base $\mathrm{x}$ motion, Test 2 Position 3

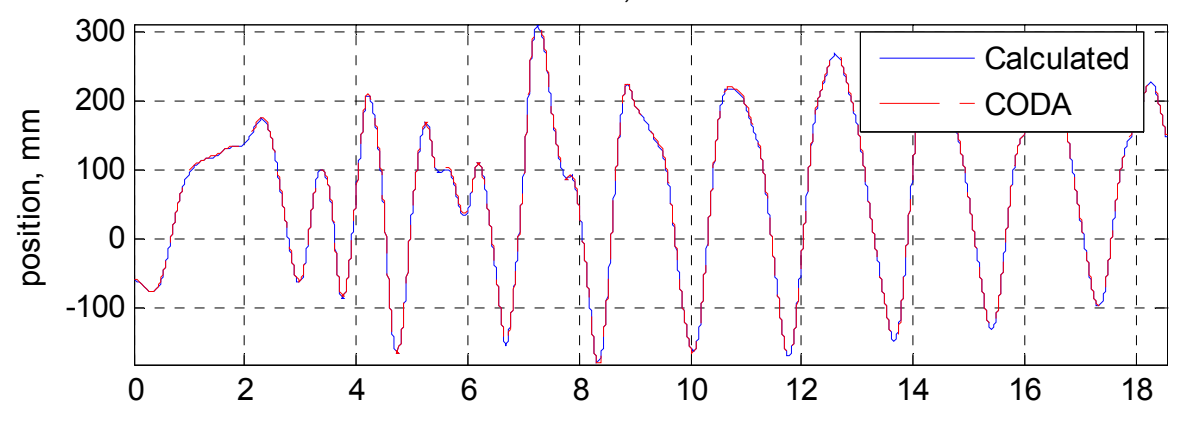

Base y motion, Test 2 Position 3

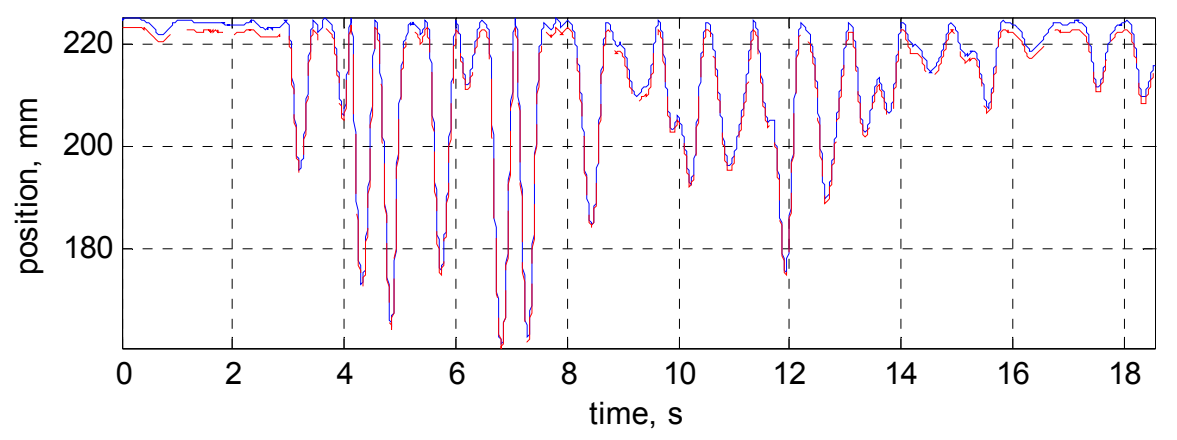

Base $\mathrm{x}$ motion, Test 2 Position 7

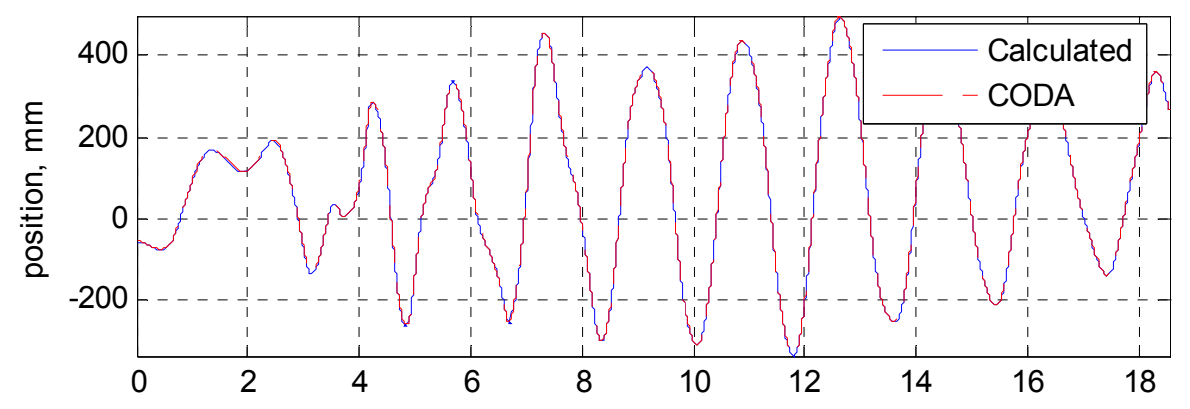

Base y motion, Test 2 Position 7

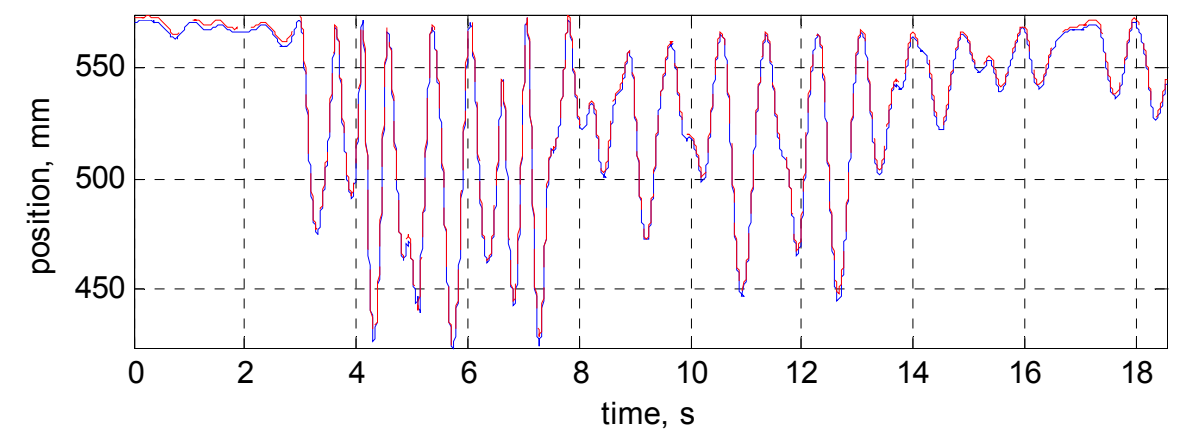


Base $\mathrm{x}$ motion, Test 2 Position 8

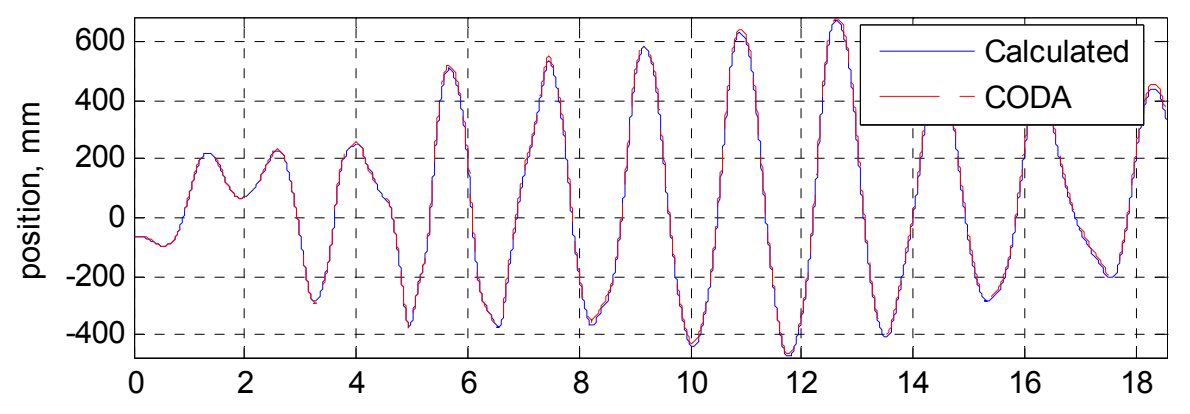

Base y motion, Test 2 Position 8
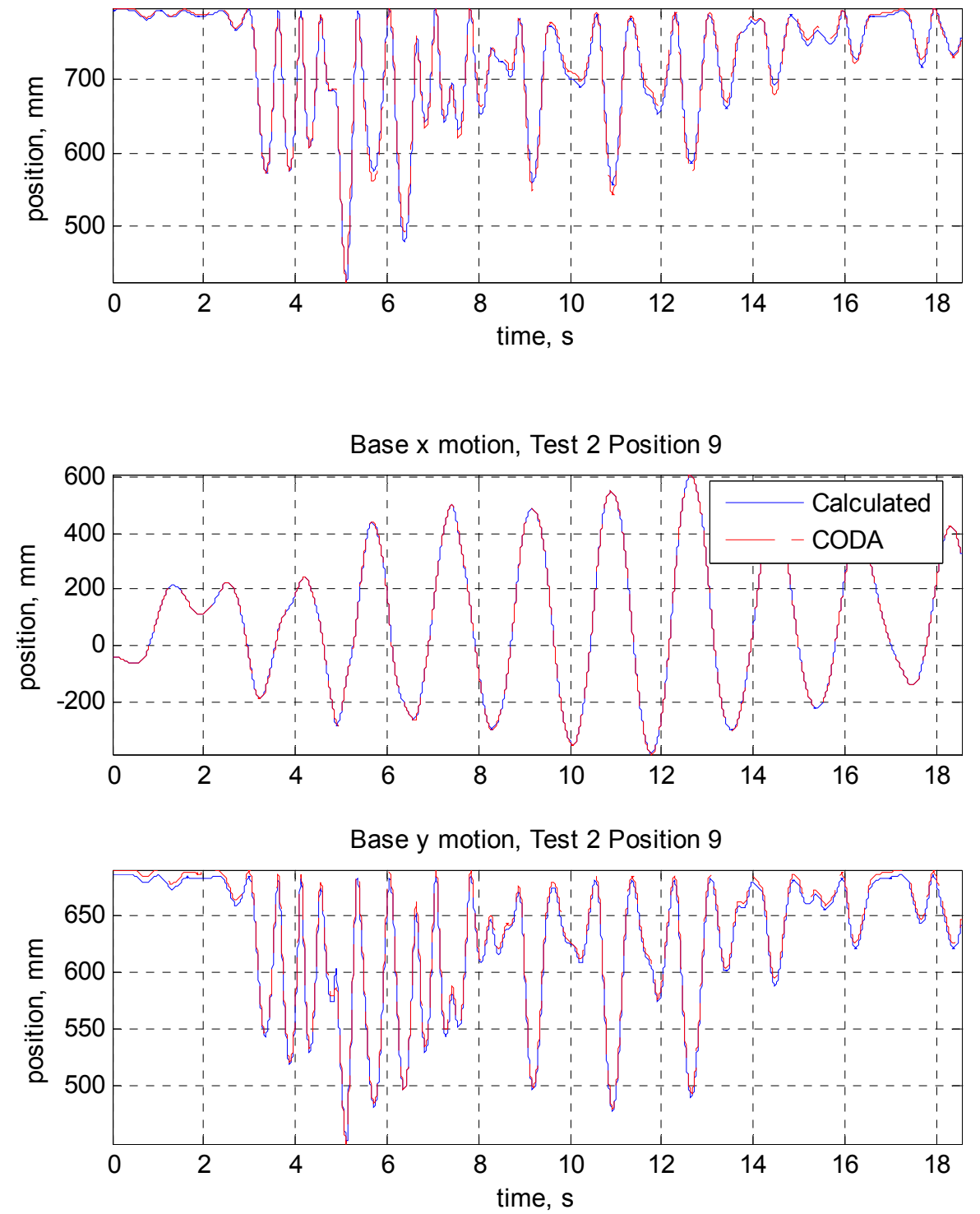
Base $\mathrm{x}$ motion, Test 3 Position 1

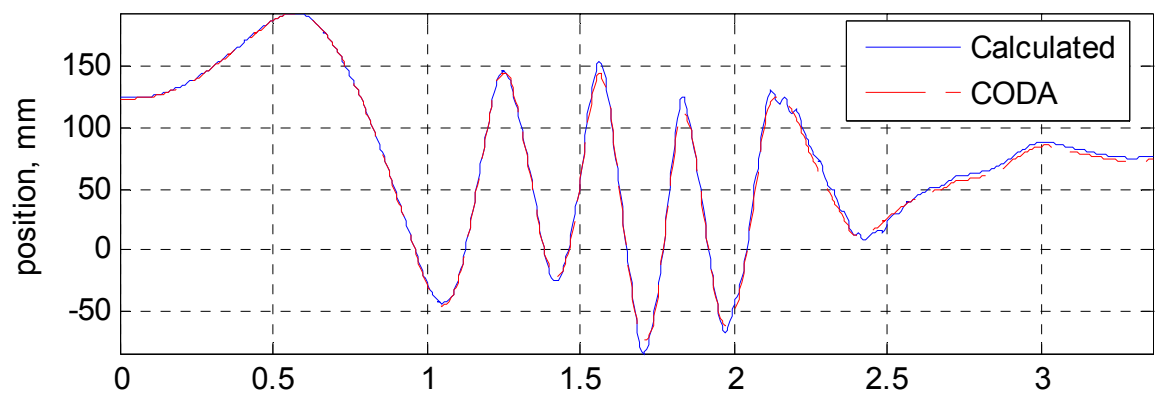

Base y motion, Test 3 Position 1
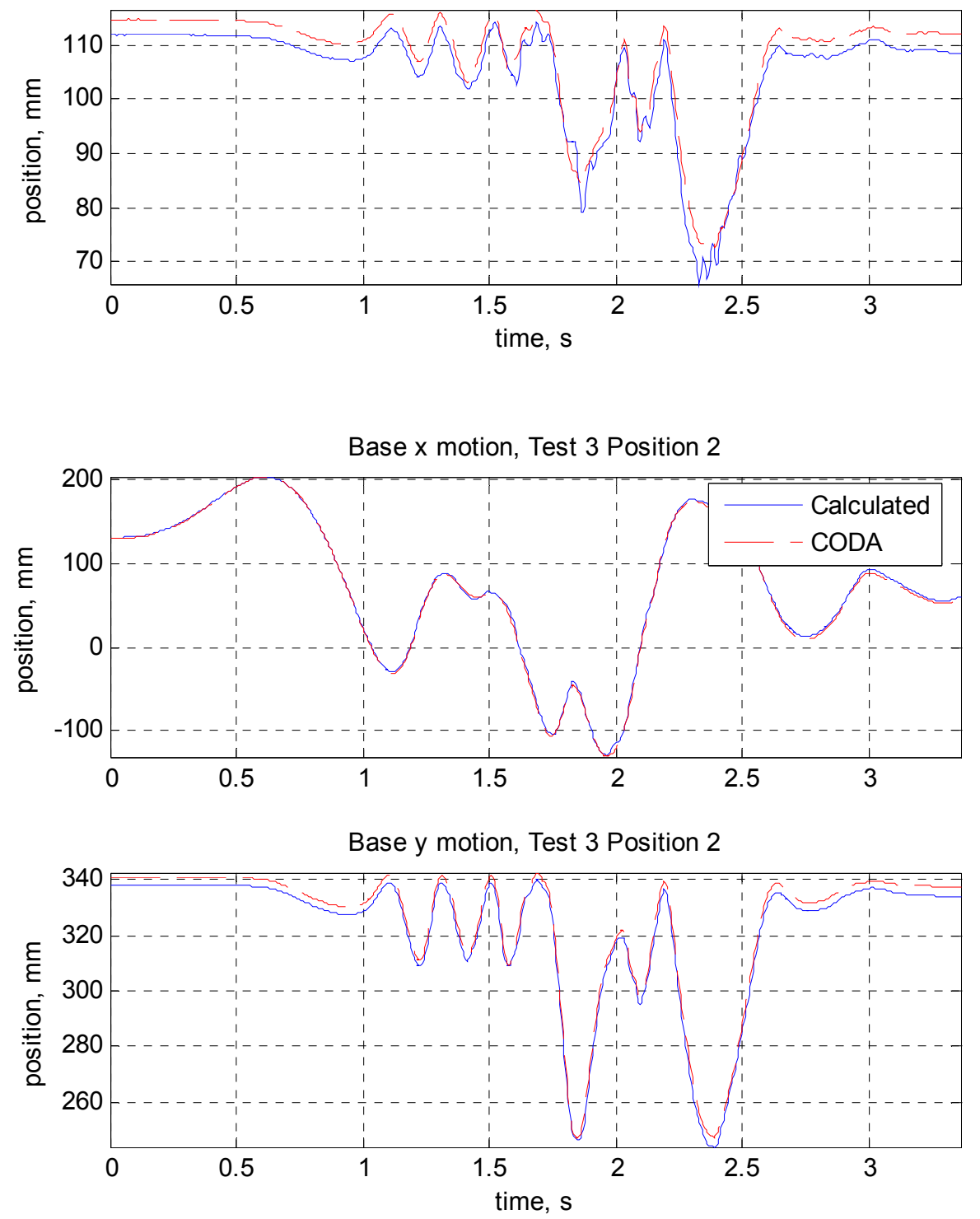
Base $\mathrm{x}$ motion, Test 3 Position 3

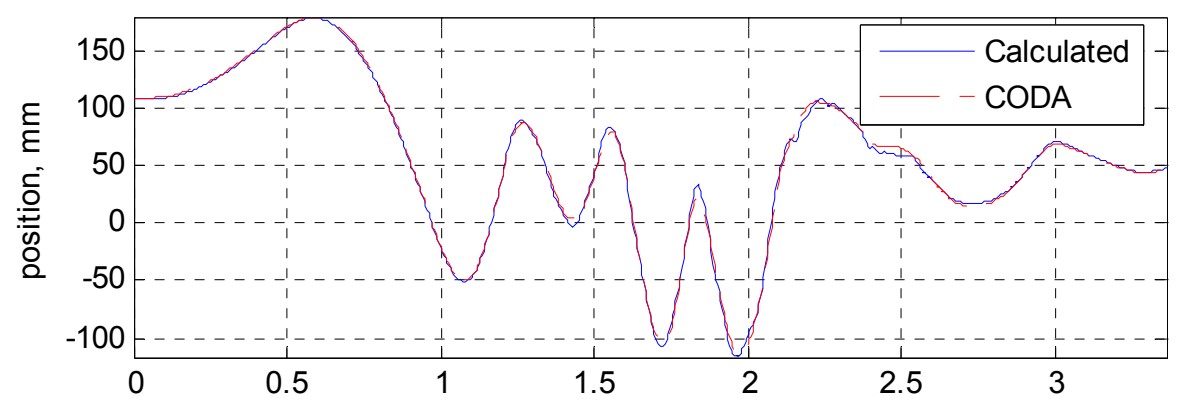

Base y motion, Test 3 Position 3

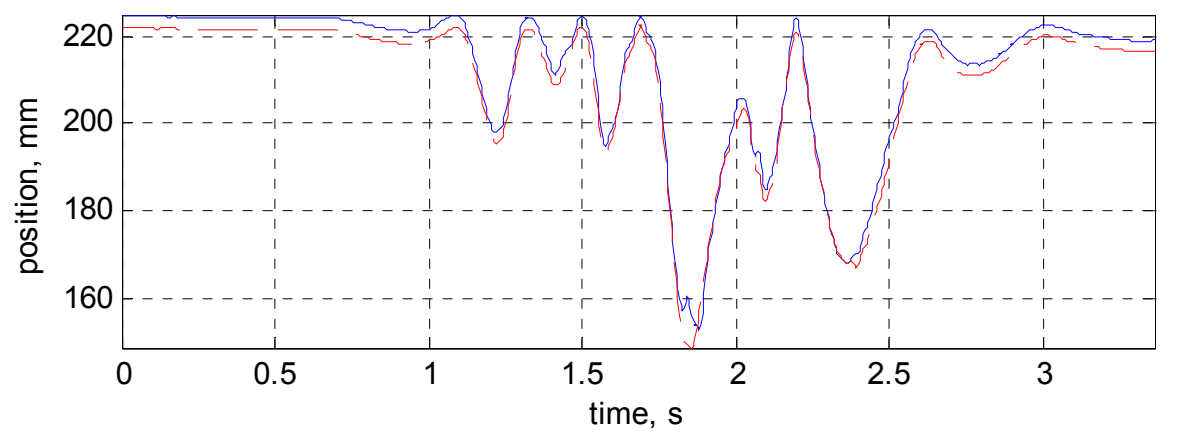

Base $\mathrm{x}$ motion, Test 3 Position 7

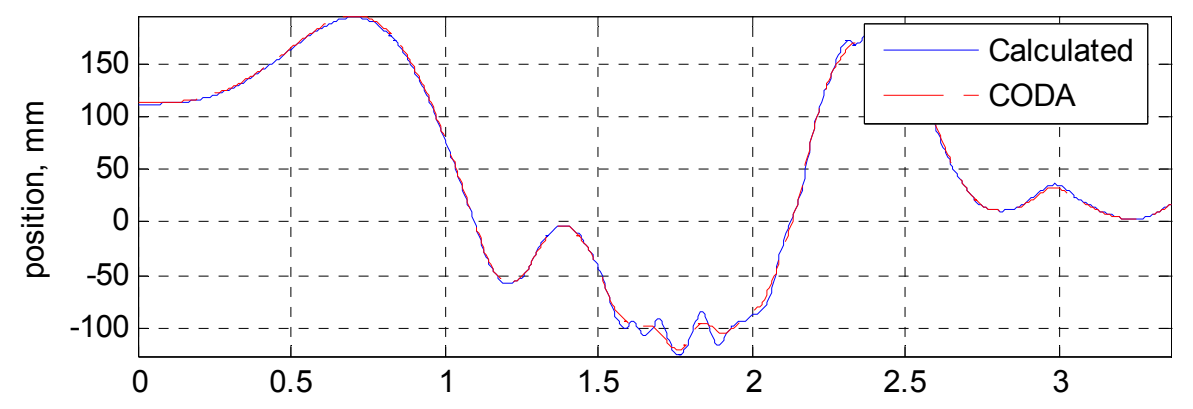

Base y motion, Test 3 Position 7

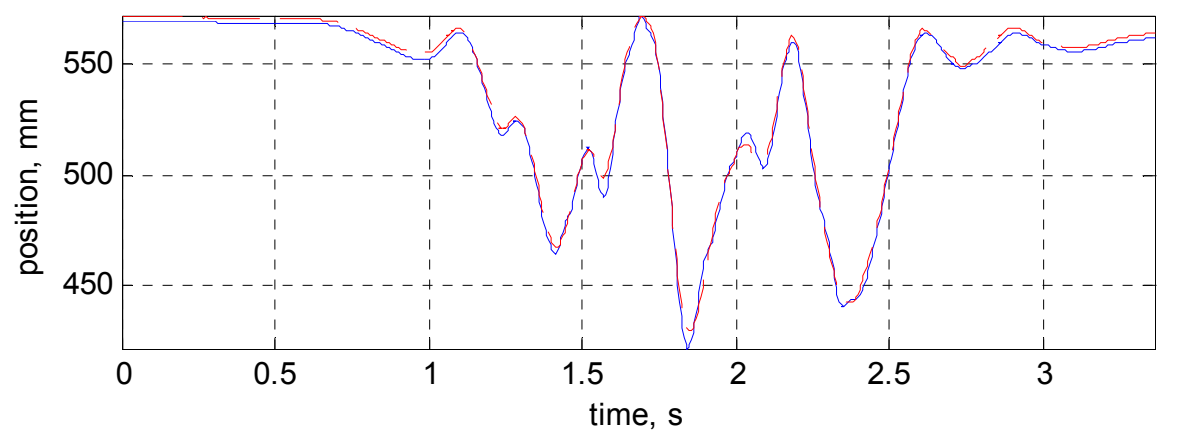


Base $\mathrm{x}$ motion, Test 3 Position 8

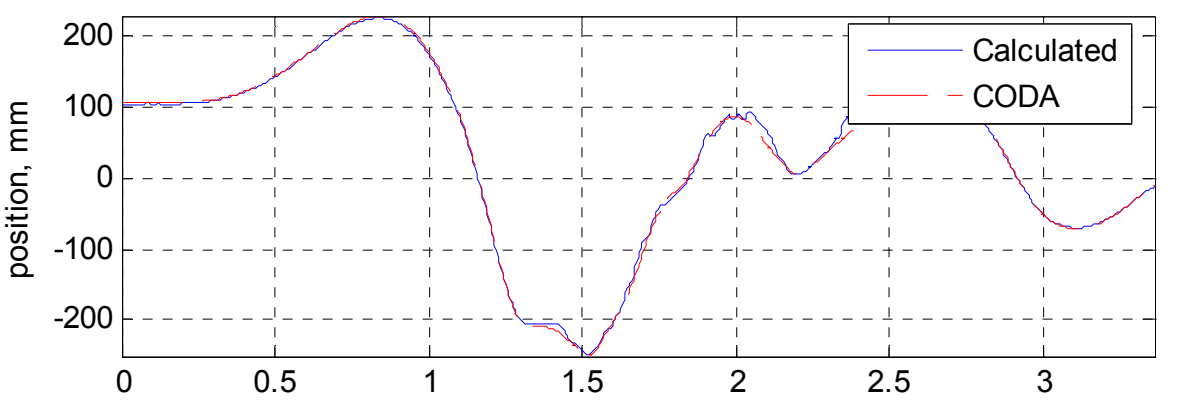

Base y motion, Test 3 Position 8

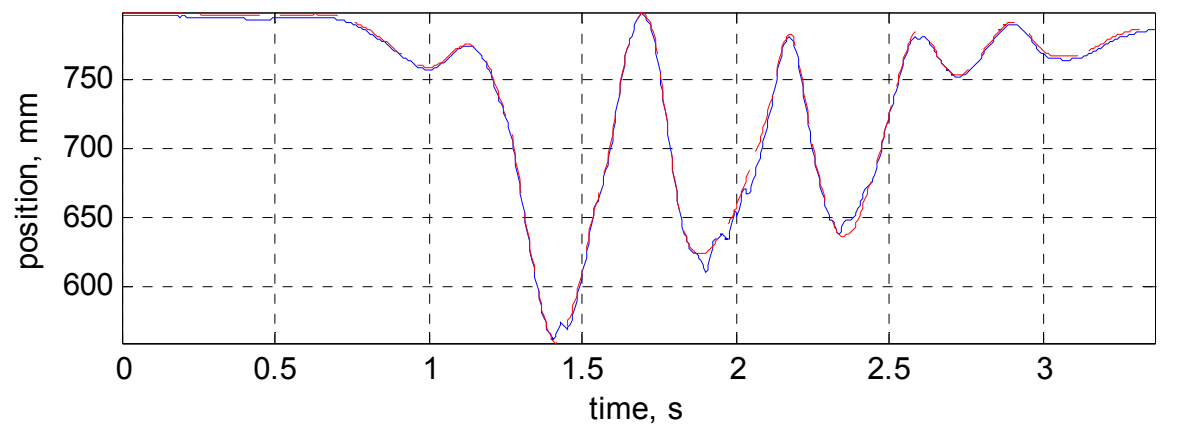

\section{Base $\mathrm{x}$ motion, Test 3 Position 9}

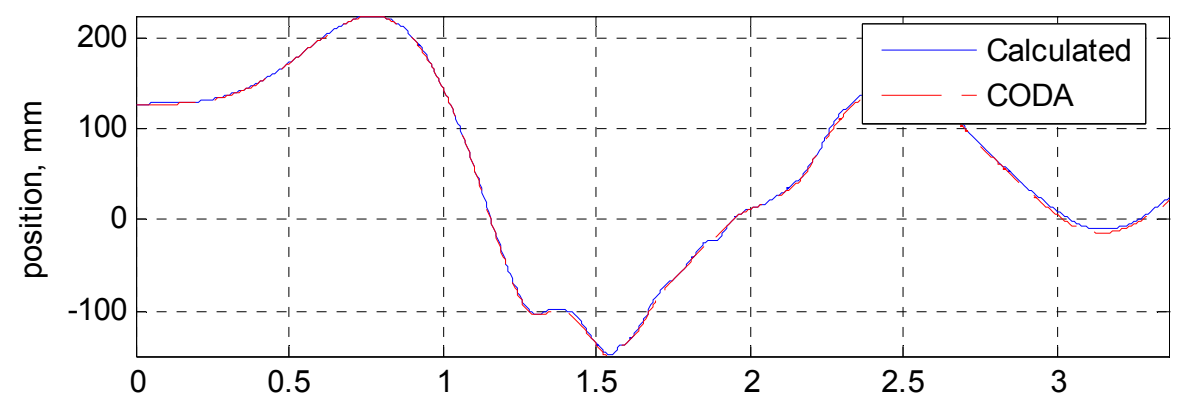

Base y motion, Test 3 Position 9

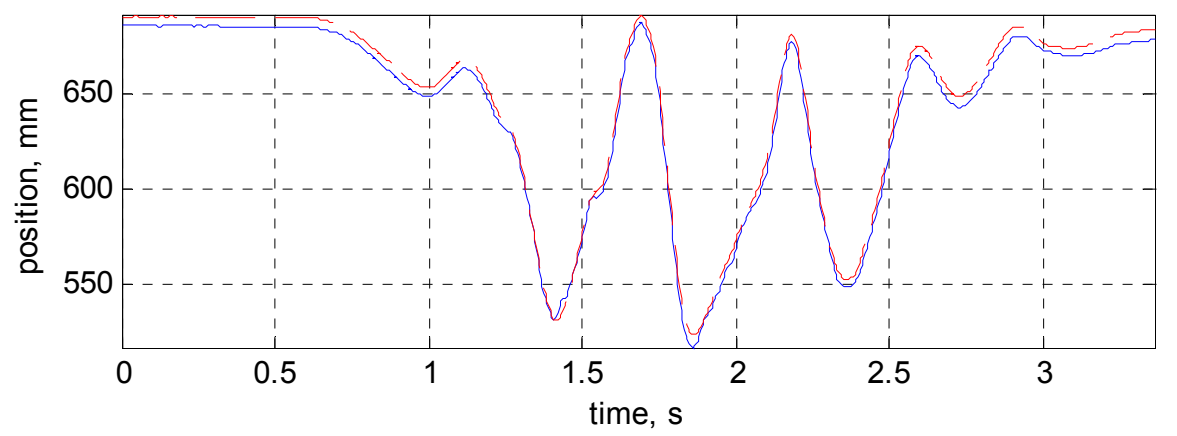


Base $\mathrm{x}$ motion, Test 4 Position 1

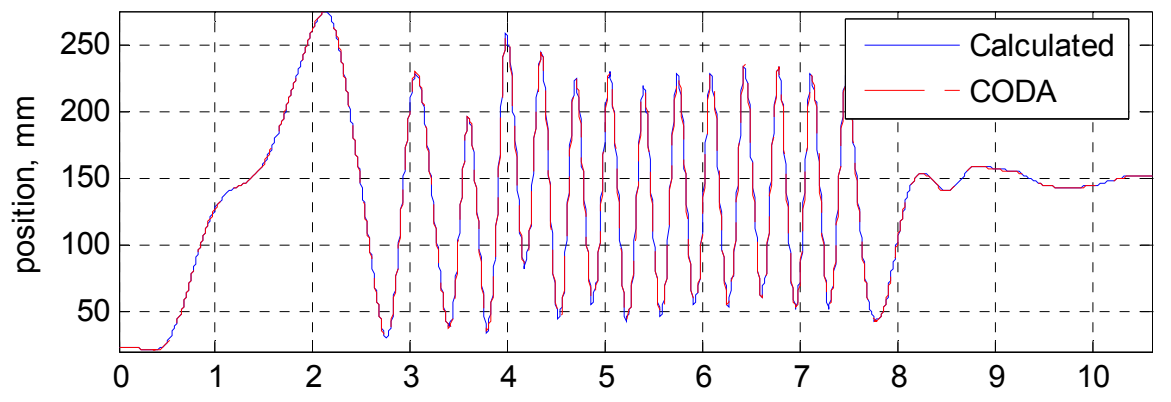

Base y motion, Test 4 Position 1

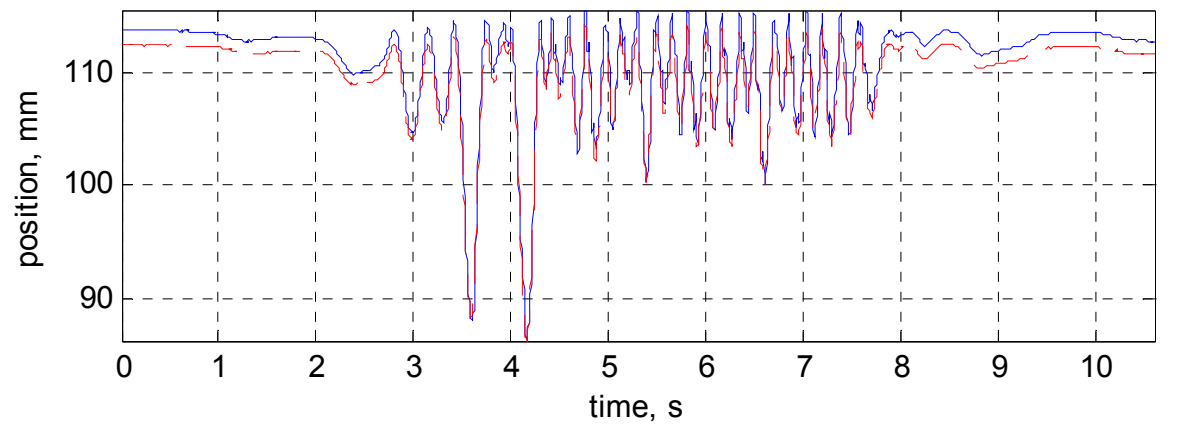

\section{Base $\mathrm{x}$ motion, Test 4 Position 2}

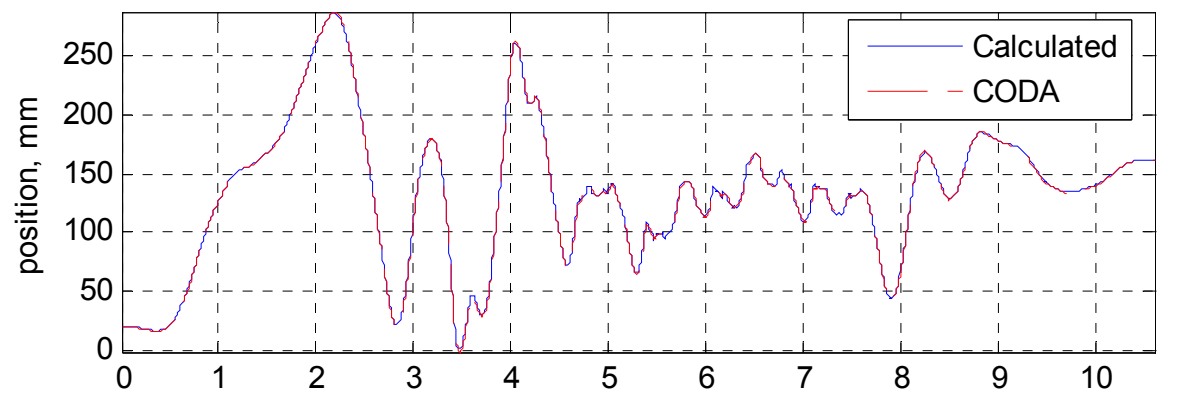

Base y motion, Test 4 Position 2

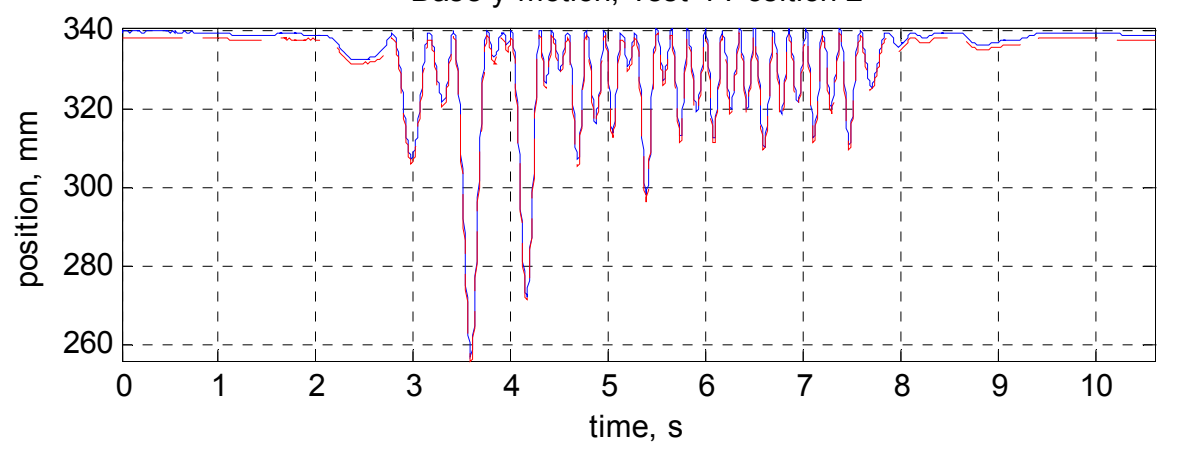


Base $\mathrm{x}$ motion, Test 4 Position 3

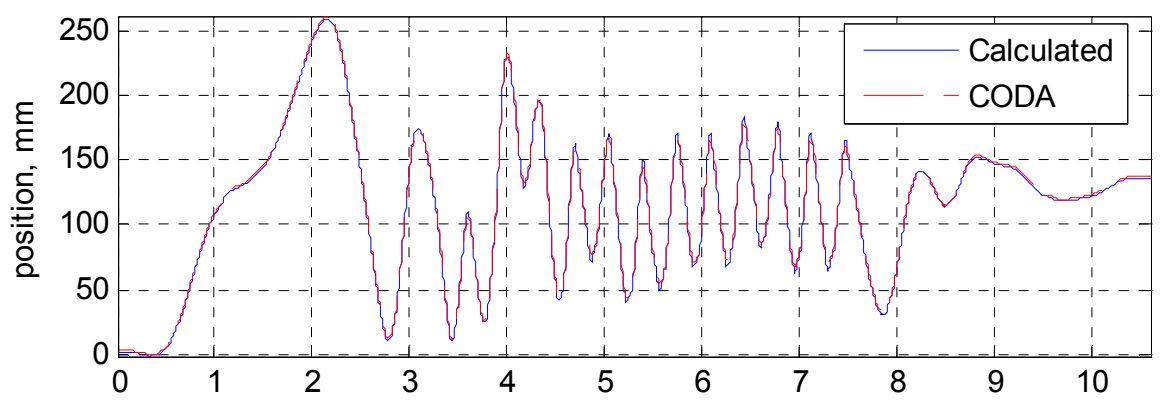

Base y motion, Test 4 Position 3

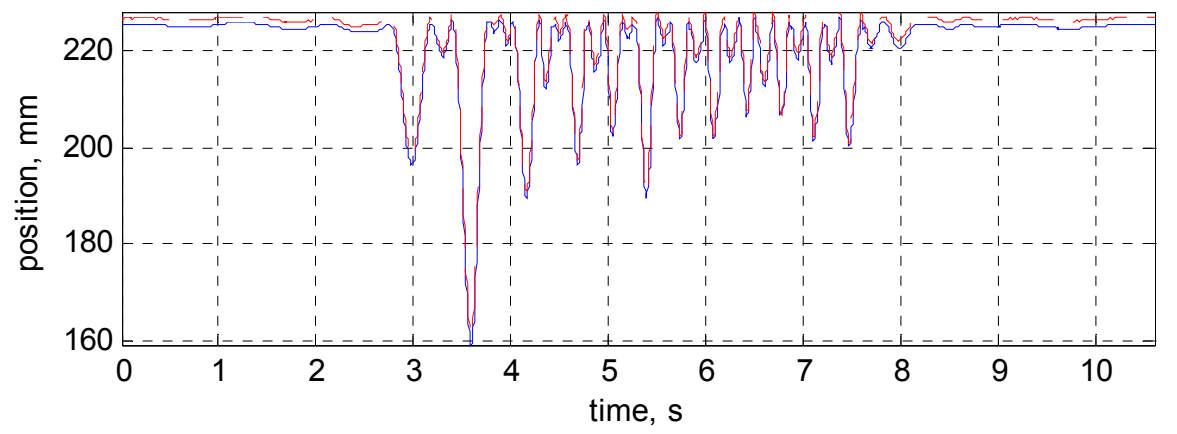

Base $\mathrm{x}$ motion, Test 4 Position 7

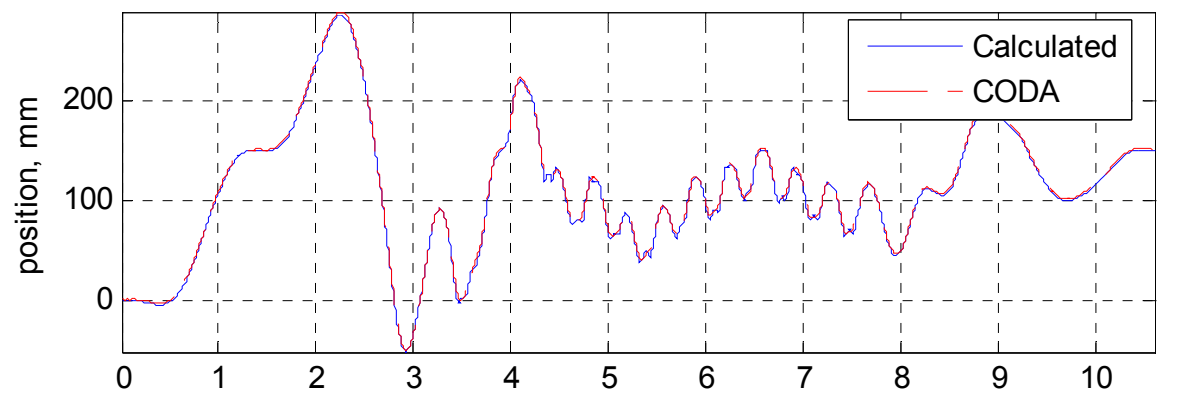

Base y motion, Test 4 Position 7

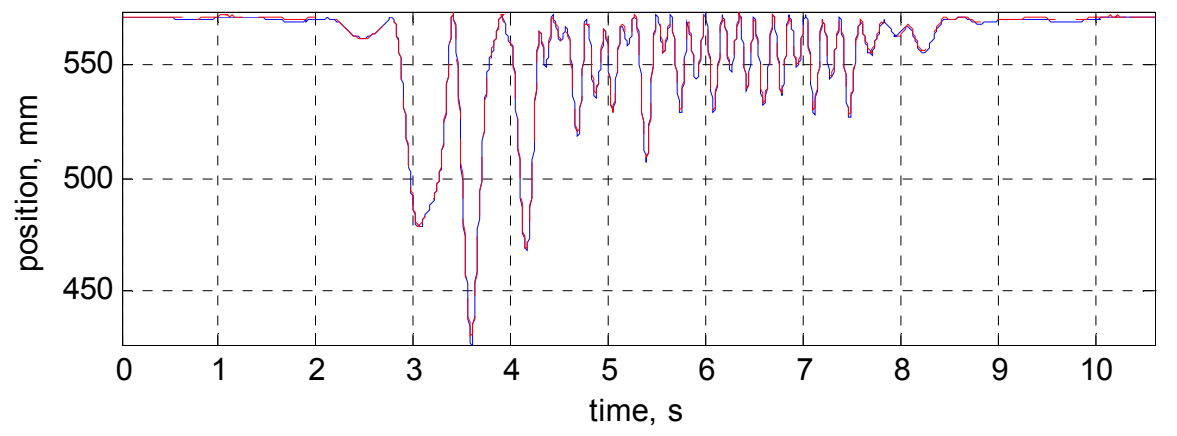


Base $\mathrm{x}$ motion, Test 4 Position 8

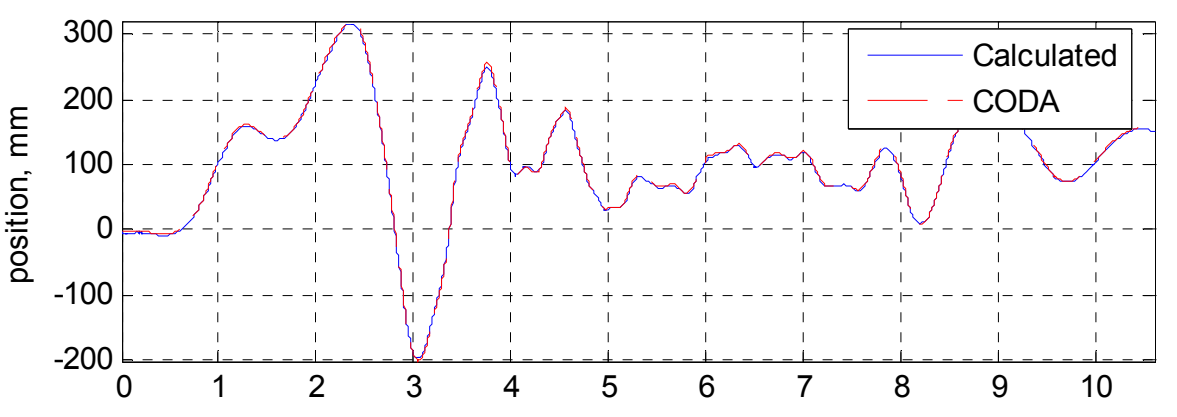

Base y motion, Test 4 Position 8

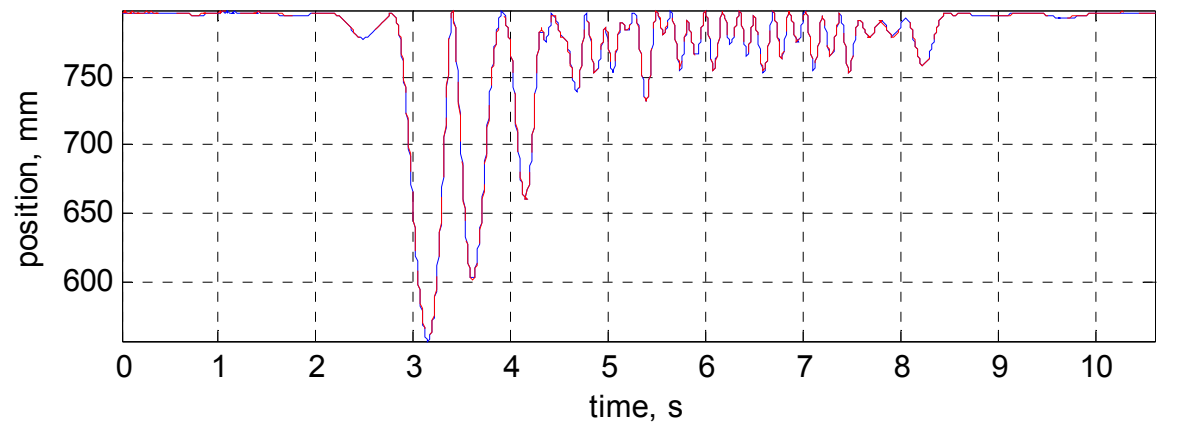

Base $\mathrm{x}$ motion, Test 4 Position 9

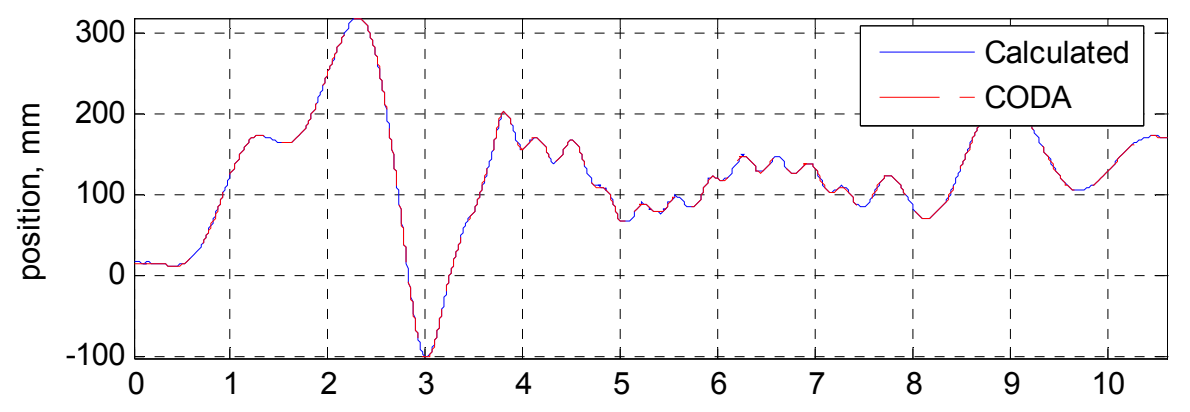

Base y motion, Test 4 Position 9

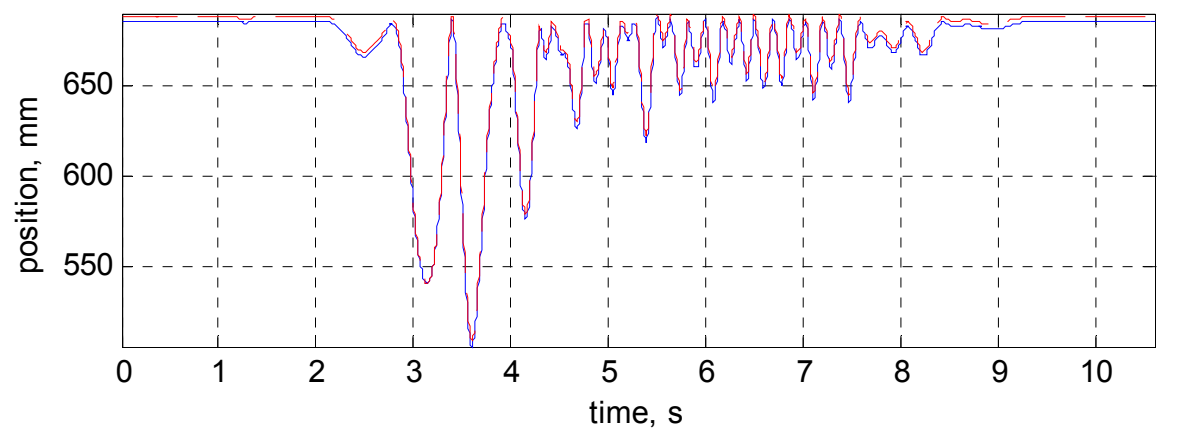




\section{Vita}

Kevin Curtis Ayer was born and raised in Baton Rouge, Louisiana, in 1979 to Fred and Joy Ayer. The youngest of three sons, and following both of his brothers' footsteps, his life revolved around a dual career of academics and athletics. He was an accomplished swimmer throughout his undergraduate years at LSU and graduated with his Bachelor of Science in Mechanical Engineering degree in May, 2002. After a brief two-year period of working, he decided to return to LSU and pursue a Master of Science in Mechanical Engineering degree focusing on biomechanics. Completing his academic requirements in December, 2006, he expects to earn his master's degree in May, 2007. 